\title{
Moderate deviations for the range of planar random walks
}

\author{
Richard Bass* Xia Chen ${ }^{\dagger}$ Jay Rosen ${ }^{\ddagger}$
}

July 3,2018

\begin{abstract}
Given a symmetric random walk in $\mathbb{Z}^{2}$ with finite second moments, let $R_{n}$ be the range of the random walk up to time $n$. We study moderate deviations for $R_{n}-\mathbb{E} R_{n}$ and $\mathbb{E} R_{n}-R_{n}$. We also derive the corresponding laws of the iterated logarithm.
\end{abstract}

\section{Introduction}

Let $X_{i}$ be symmetric i.i.d. random vectors taking values in $\mathbb{Z}^{2}$ with mean 0 and finite covariance matrix $\Gamma$, set $S_{n}=\sum_{i=1}^{n} X_{i}$, and suppose that no proper subgroup of $\mathbb{Z}^{2}$ supports the random walk $S_{n}$. For any random variable $Y$ we will use the notation

$$
\bar{Y}=Y-\mathbb{E} Y
$$

Let

$$
R_{n}=\#\left\{S_{1}, \ldots, S_{n}\right\}
$$

be the range of the random walk up to time $n$. The purpose of this paper is to obtain moderate deviation results for $\bar{R}_{n}$ and $-\bar{R}_{n}$.

*Research partially supported by NSF grant \#DMS-0244737

${ }^{\dagger}$ Research partially supported by NSF grant \#DMS-0405188.

${ }^{\ddagger}$ Research partially supported by grants from the NSF and from PSC-CUNY. 
For moderate deviations of $\bar{R}_{n}$ we have the following. Let

$$
\mathcal{H}(n)=\sum_{k=0}^{n} \mathbb{P}^{0}\left(S_{k}=0\right)
$$

Since the $X_{i}$ have two moments, then by [23], Section 2,

$$
\mathcal{H}(n)=\sum_{k=0}^{n} \mathbb{P}^{0}\left(S_{k}=0\right) \sim \frac{\log n}{2 \pi \sqrt{\operatorname{det} \Gamma}}
$$

and

$$
\mathcal{H}(n)-\mathcal{H}\left(\left[n / b_{n}\right]\right)=\sum_{k=\left[n / b_{n}\right]+1}^{n} \mathbb{P}^{0}\left(S_{k}=0\right) \sim \frac{\log b_{n}}{2 \pi \sqrt{\operatorname{det} \Gamma}}
$$

Theorem 1.1 Let $\left\{b_{n}\right\}$ be a positive sequence satisfying $b_{n} \rightarrow \infty$ and $\log b_{n}=$ $o\left((\log n)^{1 / 2}\right)$ as $n \rightarrow \infty$. There are two constants $C_{1}, C_{2}>0$ independent of the choice of the sequence $\left\{b_{n}\right\}$ such that

$$
\begin{aligned}
-C_{1} & \leq \liminf _{n \rightarrow \infty} b_{n}^{-1} \log \mathbb{P}\left\{\bar{R}_{n} \geq \frac{n}{\mathcal{H}(n)^{2}}\left(\mathcal{H}(n)-\mathcal{H}\left(\left[n / b_{n}\right]\right)\right)\right\} \\
& \leq \limsup _{n \rightarrow \infty} b_{n}^{-1} \log \mathbb{P}\left\{\bar{R}_{n} \geq \frac{n}{\mathcal{H}(n)^{2}}\left(\mathcal{H}(n)-\mathcal{H}\left(\left[n / b_{n}\right]\right)\right\} \leq-C_{2} .\right.
\end{aligned}
$$

Remark 1.2 The proof will show that $C_{2}$ in the statement of Theorem 1.1] is equal to the constant $L$ given in Theorem 1.3 in [2]. We believe that $C_{1}$ is also equal to $L$, but we do not have a proof of this fact.

A more precise statement than Theorem 1.1 is possible when the $X_{i}$ have slightly more than two moments.

Corollary 1.3 Suppose $\mathbb{E}\left[\left|X_{i}\right|^{2}\left(\log ^{+}\left(\left|X_{i}\right|\right)\right)^{\frac{1}{2}+\delta}\right]<\infty$ for some $\delta>0$. Let $\left\{b_{n}\right\}$ be a positive sequence satisfying $b_{n} \rightarrow \infty$ and $\log b_{n}=o\left((\log n)^{1 / 2}\right)$ as $n \rightarrow \infty$. There are two constants $C_{1}, C_{2}>0$ independent of the choice of the sequence $\left\{b_{n}\right\}$ such that

$$
\begin{aligned}
-C_{1} & \leq \liminf _{n \rightarrow \infty} b_{n}^{-\theta} \log \mathbb{P}\left\{\bar{R}_{n} \geq 2 \theta \pi \sqrt{\operatorname{det} \Gamma} \frac{n}{(\log n)^{2}} \log b_{n}\right\} \\
& \leq \limsup _{n \rightarrow \infty} b_{n}^{-\theta} \log \mathbb{P}\left\{\bar{R}_{n} \geq 2 \theta \pi \sqrt{\operatorname{det} \Gamma} \frac{n}{(\log n)^{2}} \log b_{n}\right\} \leq-C_{2}
\end{aligned}
$$

for any $\theta>0$. 
Remark 1.4 The constants $C_{1}, C_{2}$ are the same as in the statement of Theorem 1.1. See Remark 1.2.

For $b_{n}$ tending to infinity faster than the rate given in Theorem 1.1, e.g., $\log b_{n}=(\log n)^{2}$, then we are in the realm of large deviations. For results on large deviations of the range, see [14], [18], [19].

For the moderate deviations of $-\bar{R}_{n}=\mathbb{E} R_{n}-R_{n}$ we have the following. Let $\kappa(2,2)$ be the smallest $A$ such that

$$
\|f\|_{4} \leq A\|\nabla f\|_{2}^{1 / 2}\|f\|_{2}^{1 / 2}
$$

for all $f \in C^{1}$ with compact support. (This constant appeared in [2].)

Theorem 1.5 Suppose $b_{n} \rightarrow \infty$ and $b_{n}=o\left((\log n)^{1 / 5}\right)$ as $n \rightarrow \infty$. For $\lambda>0$

$$
\lim _{n \rightarrow \infty} \frac{1}{b_{n}} \log \mathbb{P}\left(-\bar{R}_{n}>\lambda \frac{n b_{n}}{\log ^{2} n}\right)=-(2 \pi)^{-2}(\operatorname{det} \Gamma)^{-1 / 2} \kappa(2,2)^{4} \lambda .
$$

Comparing Theorems 1.1 and 1.5, we see that the upper and lower tails of $\bar{R}_{n}$ are quite different. This is similar to the behavior of the distribution of the self-intersection local time of planar Brownian motion. This is not surprising, since LeGall, [21, Theorem 6.1], shows that $\bar{R}_{n}$, properly normalized, converges in distribution to the self-intersection local time.

The moderate deviations of $\bar{R}_{n}$ are quite similar in nature to those of $-\bar{L}_{n}$, where $L_{n}$ is the number of self-intersections of the random walk $S_{n}$; see 4 . Again, 21, Theorem 6.1] gives a partial explanation of this. However the case of the range is much more difficult than the corresponding results for intersection local times. The latter case can be represented as a quadratic functional of the path, which is amenable to the techniques of large deviation theory, while the range cannot be so represented. This has necessitated the development of several new tools, see in particular Sections 5 and 6] which we expect will have further applications in the study of the range of random walks.

Theorem 1.1 gives rise to the following LIL for $\bar{R}_{n}$. 


\section{Theorem 1.6}

$$
\limsup _{n \rightarrow \infty} \frac{\bar{R}_{n}}{n \log \log \log n / \log ^{2} n}=2 \pi \sqrt{\operatorname{det} \Gamma}, \quad \text { a.s. }
$$

This result is an improvement of that in [6]; there it was required that the $X_{i}$ be bounded random variables and the constant was not identified. Theorem 1.1 is a more precise estimate than is needed for Theorem [1.6 this

is why Theorem 1.1 needs to be stated in terms of $\mathcal{H}(n)$ while Theorem 1.6 does not.

For an LIL for $-\bar{R}_{n}$ we have a different rate.

Theorem 1.7 We have

$$
\limsup _{n \rightarrow \infty} \frac{-\bar{R}_{n}}{n \log \log n / \log ^{2} n}=(2 \pi)^{-2} \sqrt{\operatorname{det} \Gamma} \kappa(2,2)^{4}, \quad \text { a.s. }
$$

The study of the range of a lattice-valued (or $\mathbb{Z}^{d}$-valued) random walk has a long history in probability and the results show a strong dependence on the dimension $d$. See [15], 20], 21], [23], [18, [19, [14], and [6] and the references in these papers, to cite only a few. The two dimensional case seems to be the most difficult; in one dimension no renormalization is needed (see [9]), while for $d \geq 3$ the tails are sub-Gaussian and have asymptotically symmetric behavior. In two dimensions, renormalization is needed and the tails have non-symmetric behavior. In this case, the central limit theorem was proved in 1986 in [21], while the first law of the iterated logarithm was not proved until a few years ago in [6].

Acknowledgment: We would like to thank Greg Lawler and Takashi Kumagai for helpful discussions and their interest in this paper.

\section{Moments of the range}

In this section we first give an estimate for the expectation of the range.

By [23], Theorem 6.9, we have

$$
\mathbb{E} R_{n}=\frac{n}{\mathcal{H}(n)}+\frac{1}{2 \pi \sqrt{\operatorname{det} \Gamma}} \frac{n}{\mathcal{H}(n)^{2}}(1+o(1)),
$$


where $\mathcal{H}$ is defined in (1.2). By [23], Section 2,

$$
\mathcal{H}(n) \sim \frac{\log n}{2 \pi \sqrt{\operatorname{det} \Gamma}}
$$

and

$$
\mathcal{H}(n)-\mathcal{H}(m) \sim \frac{\log (n / m)}{2 \pi \sqrt{\operatorname{det} \Gamma}}
$$

as $n$ and $m$ tend to infinity.

Throughout this paper we will mostly be concerned with random walks that have only second moments. The exception is the following proposition, which supposes slightly more than two moments, and Corollary 1.3.

Proposition 2.1 Suppose $\left\{X_{i}\right\}$ is a sequence of i.i.d. mean zero random vectors taking values in $\mathbb{Z}^{2}$ with

$$
\mathbb{E}\left(|X|^{2}\left(\log ^{+}|X|\right)^{\frac{1}{2}+\delta}\right)<\infty
$$

for some $\delta>0$ and nondegenerate covariance matrix $\Gamma$. Let $S_{n}=\sum_{i=1}^{n} X_{i}$ and suppose $S_{n}$ is strongly aperiodic. Then

$$
\mathbb{P}\left(S_{n}=0\right)=\frac{1}{2 \pi n \sqrt{\operatorname{det} \Gamma}}+O\left(\frac{1}{n(\log n)^{(1+\delta) / 2}}\right)
$$

Proof. Let $\varphi$ be the characteristic function of $X_{i}$, let $x \cdot y$ denote the inner product in $\mathbb{R}^{2}$, let $Q(u)=u \cdot \Gamma u$, and let $C=[-\pi, \pi]^{2}$. We observe that

$$
\begin{aligned}
\mid 1-\varphi(u) & -Q(u) \mid \\
& =\left|\mathbb{E}\left(1-e^{i u \cdot X}+i u \cdot X+(1 / 2)(i u \cdot X)^{2}\right)\right| \\
& \leq c_{1}|u|^{3} \mathbb{E}\left(1_{\{|X| \leq 1 /|u|\}}|X|^{3}\right)+c_{1}|u|^{2} \mathbb{E}\left(1_{\{|X|>1 /|u|\}}|X|^{2}\right)
\end{aligned}
$$


and consequently for any fixed $M>0$

$$
\begin{aligned}
& |1-\varphi(u / \sqrt{n})-Q(u / \sqrt{n})| \\
& \leq c_{2}\left(\frac{1}{n^{3 / 2}}\right) \mathbb{E}\left(1_{\{|u||X| \leq \sqrt{n}\}}(|u||X|)^{3}\right)+c_{2}\left(\frac{1}{n}\right) \mathbb{E}\left(1_{\{|u||X|>\sqrt{n}\}}(|u||X|)^{2}\right) \\
& \leq c_{3} \frac{1}{n^{3 / 2}}+c_{3}\left(\frac{1}{n^{3 / 2}}\right) \mathbb{E}\left(1_{\{M<|u||X| \leq \sqrt{n}\}}(|u||X|)^{3}\right) \\
& +c_{3}\left(\frac{1}{n}\right) \mathbb{E}\left(1_{\{|u||X|>\sqrt{n}\}}(|u||X|)^{2}\right) .
\end{aligned}
$$

Choose $\mathrm{M}$ so that $x / \log ^{1 / 2+\delta}(x)$ is monotone increasing on $x \geq M$, and therefore

$$
\begin{aligned}
& \mathbb{E}\left(1_{\{M<|u||X| \leq \sqrt{n}\}}(|u||X|)^{3}\right) \\
& \quad \leq \mathbb{E}\left(1_{\{M<|u||X| \leq \sqrt{n}\}}(|u||X|)^{2} \log ^{1 / 2+\delta}(|u||X|) \frac{|u||X|}{\log ^{1 / 2+\delta}(|u||X|)}\right) \\
& \quad \leq\left(\frac{\sqrt{n}}{\log ^{1 / 2+\delta}(\sqrt{n})}\right) \mathbb{E}\left(1_{\{M<|u||X| \leq \sqrt{n}\}}(|u||X|)^{2} \log ^{1 / 2+\delta}(|u||X|)\right) .
\end{aligned}
$$

Also

$$
\begin{aligned}
& \mathbb{E}\left(1_{\{|u||X|>\sqrt{n}\}}(|u||X|)^{2}\right) \\
& \quad \leq\left(\frac{1}{\log ^{1 / 2+\delta}(\sqrt{n})}\right) \mathbb{E}\left(1_{\{|u||X|>\sqrt{n}\}}(|u||X|)^{2} \log ^{1 / 2+\delta}(|u||X|)\right) .
\end{aligned}
$$

(2.7) then implies that

$$
|1-\varphi(u / \sqrt{n})-Q(u / \sqrt{n})| \leq c \frac{|u|^{2}\left|\log ^{1 / 2+\delta}(|u|)\right|}{n \log ^{1 / 2+\delta}(n)} .
$$

Following the proof in Spitzer [34], pp. 76-77,

$$
\begin{aligned}
2 \pi n \mathbb{P}\left(S_{n}=0\right) & =(2 \pi)^{-1} \int_{\sqrt{n} C} \varphi(u / \sqrt{n})^{n} d u \\
& =I_{0}+I_{1}\left(n, A_{n}\right)+I_{2}\left(n, A_{n}\right)+I_{3}\left(n, A_{n}, r\right)+I_{4}(n, r),
\end{aligned}
$$


where

$$
\begin{aligned}
I_{0} & =(2 \pi)^{-1} \int_{\mathbb{R}^{2}} e^{-Q(u) / 2} d u=(\operatorname{det} Q)^{-1 / 2}, \\
I_{1}\left(n, A_{n}\right) & =(2 \pi)^{-1} \int_{|u| \leq A_{n}}\left[\varphi(u / \sqrt{n})^{n}-e^{-Q(u) / 2}\right] d u, \\
I_{2}\left(n, A_{n}\right) & =-(2 \pi)^{-1} \int_{|u|>A_{n}} e^{-Q(u) / 2} d u, \\
I_{3}\left(n, A_{n}, r\right) & =(2 \pi)^{-1} \int_{A_{n}<|u|<r \sqrt{n}} \varphi(u / \sqrt{n})^{n} d u, \\
I_{4}(n, r) & =(2 \pi)^{-1} \int_{|u| \geq r \sqrt{n}, u \in \sqrt{n} C} \varphi(u / \sqrt{n})^{n} d u .
\end{aligned}
$$

Still following [34, we can choose $r$ such that $\left|\varphi(u / \sqrt{n})^{n}\right| \leq e^{-Q(u) / 4}$ if $|u| \leq$ $r \sqrt{n}$ and by the strong aperiodicity there exists $\gamma>0$ such that $|\varphi(u / \sqrt{n})| \leq$ $1-\gamma$ if $|u|>r \sqrt{n}$ and $u \in \sqrt{n} C$. Set $A_{n}=c_{4} \sqrt{\log \log n}$. We have

$$
\left|I_{4}(n, r)\right| \leq(2 \pi)^{-1} \int_{u \in \sqrt{n} C}(1-\gamma)^{n} d u=O\left(n^{-p}\right)
$$

for every positive integer $p$. Next

$$
\left|I_{3}\left(n, A_{n}, r\right)\right| \leq \int_{|u|>c_{4} \sqrt{\log \log n}} e^{-Q(u) / 4} d u=O\left((\log n)^{-2}\right)
$$

for $c_{4}$ large and similarly we have the same bound for $\left|I_{2}\left(n, A_{n}\right)\right|$. To estimate $I_{1}\left(n, A_{n}\right)$ we use the inequality $\left|a^{n}-b^{n}\right| \leq n|a-b|$ if $|a|,|b| \leq 1$ with $a=$ $\varphi(u / \sqrt{n})$ and $b=e^{-Q(u) / 2 n}$. Using (2.10) and the analogous expansion for $e^{-Q(u) / 2 n}$ we have

$$
\begin{aligned}
\left|\varphi(u / \sqrt{n})^{n}-e^{-Q(u) / 2}\right| & \leq n\left|\varphi(u / \sqrt{n})-e^{-Q(u) / 2 n}\right| \\
& \leq c_{5} n \frac{|u|^{2}\left|\log ^{1 / 2+\delta}(|u|)\right|}{n \log ^{1 / 2+\delta}(n)}=c_{5} \frac{|u|^{2}\left|\log ^{1 / 2+\delta}(|u|)\right|}{\log ^{1 / 2+\delta}(n)} .
\end{aligned}
$$

Integrating this over the set $\left\{|u| \leq A_{n}\right\}$, we see

$$
\left|I_{1}\left(n, A_{n}\right)\right|=O\left((\log \log n)^{2+\delta / 2} /(\log n)^{1 / 2+\delta}\right)=O\left(1 /(\log n)^{(1+\delta) / 2}\right) .
$$

Summing $I_{0}$ through $I_{4}$, we obtain

$$
2 \pi n \mathbb{P}\left(S_{n}=0\right)=(\operatorname{det} \Gamma)^{-1 / 2}+O\left(1 /(\log n)^{(1+\delta) / 2}\right) .
$$


Next we establish some sharp exponential estimates for the range and intersection of ranges. Aside from their intrinsic interest, they will be used to estimate the tail probabilities in our first main theorem.

We write $S(I)$ for $\left\{S_{k}: k \in I\right\}$. Let $S^{(i)}, i=1, \ldots, p$ be $p$ independent copies of $S$. First, by Corollary 1 of [8], for any integers $a \geq 1, n_{1}, \cdots n_{a} \geq 1$,

$$
\left(\mathbb{E} J_{n_{1}+\cdots+n_{a}}^{m}\right)^{1 / p} \leq \sum_{\substack{k_{1}+\cdots+k_{a}=m \\ k_{1}, \cdots, k_{a} \geq 0}} \frac{m !}{k_{1} ! \cdots k_{a} !}\left(\mathbb{E} J_{n_{1}}^{k_{1}}\right)^{1 / p} \cdots\left(\mathbb{E} J_{n_{a}}^{k_{a}}\right)^{1 / p}
$$

where

$$
J_{n}=\#\left\{S^{(1)}[1, n] \cap \cdots \cap S^{(p)}[1, n]\right\} \quad n=1,2, \cdots .
$$

In the next Theorem we deduce from this the exponential integrability of $J_{n}$, which was established in [6 in the special case $p=2$ and under the condition that $S$ had bounded increments.

Theorem 2.2 Assume that the planar random walk $S$ has finite second moments and zero mean. There exists $\theta>0$ such that

$$
\sup _{n} \sup _{y_{1}, \cdots, y_{p}} \mathbb{E}^{\left(y_{1}, \cdots, y_{p}\right)} \exp \left\{\theta\left(\frac{(\log n)^{p}}{n}\right)^{1 /(p-1)} J_{n}^{1 /(p-1)}\right\}<\infty .
$$

Proof. We recall the fact (see Remarks, p. 664, in [23]) that

$$
\mathbb{E} J_{n}^{k} \leq(k !)^{p}\left(\mathbb{E} J_{n}\right)^{k}, \quad k=0,1, \cdots,
$$

and for some $C<\infty$

$$
\mathbb{E} J_{n} \leq \frac{C n}{(\log n)^{p}}, \quad n=1, \cdots .
$$

The proof of (2.12) is a modification of the approach used in Lemma 1 of [8]. We begin by showing that there is a constant $C>0$ such that

$$
\sup _{n} \mathbb{E} J_{n}^{m} \leq C^{m}(m !)^{p-1}\left(\frac{n}{(\log n)^{p}}\right)^{m}, \quad m, n=1,2, \cdots .
$$


We first consider the case $m \leq(\log n)^{(p-1) / p}$. Write $l(n, m)=[n / m]+1$. Then by (2.11) and (2.14),

$$
\begin{aligned}
\left(\mathbb{E} J_{n}^{m}\right)^{1 / p} & \leq \sum_{\substack{k_{1}+\cdots+k_{m}=m \\
k_{1}, \cdots, k_{m} \geq 0}} \frac{m !}{k_{1} ! \cdots k_{m} !}\left(\mathbb{E} J_{l(n, m)}^{k_{1}}\right)^{1 / p} \cdots\left(\mathbb{E} J_{l(n, m)}^{k_{m}}\right)^{1 / p} \\
& \leq \sum_{\substack{k_{1}+\cdots+k_{m}=m \\
k_{1}, \cdots, k_{m} \geq 0}} \frac{m !}{k_{1} ! \cdots k_{m} !} k_{1} ! \cdots k_{m} !\left(\mathbb{E} J_{l(n, m)}\right)^{k_{1} / p} \cdots\left(\mathbb{E} J_{l(n, m)}\right)^{k_{m} / p} \\
& =\left(\begin{array}{c}
2 m-1 \\
m
\end{array}\right) m !\left(\mathbb{E} J_{l(n, m)}\right)^{m / p} \leq\left(\begin{array}{c}
2 m-1 \\
m
\end{array}\right) m ! C^{m}\left(\frac{(n / m)}{(\log n)^{p}}\right)^{m / p} \\
& \leq\left(\begin{array}{c}
2 m \\
m
\end{array}\right)(m !)^{\frac{p-1}{p}} C^{m}\left(\frac{n}{(\log n)^{p}}\right)^{m / p}
\end{aligned}
$$

where the second inequality follows from (2.13) and the third from (2.14) using the fact that $m=O(\log n)$ so that $\log n=O(\log (n / m))$. Hence, taking $p$-th powers we obtain

$$
\mathbb{E} J_{n}^{m} \leq\left(\begin{array}{c}
2 m \\
m
\end{array}\right)^{p} C^{p m}(m !)^{p-1}\left(\frac{n}{(\log n)^{p}}\right)^{m},
$$

and (2.15) for the case of $m \leq(\log n)^{(p-1) / p}$ follows from the fact

$$
\left(\begin{array}{c}
2 m \\
m
\end{array}\right) \leq 4^{m}
$$

For the case $m>(\log n)^{(p-1) / p}$, notice from the definition of $J_{n}$ that $J_{n} \leq n$. So we have

$$
\begin{aligned}
\mathbb{E} J_{n}^{m} \leq n^{m} & =(\log n)^{p m}\left(\frac{n}{(\log n)^{p}}\right)^{m} \leq m^{(p-1) m}\left(\frac{n}{(\log n)^{p}}\right)^{m} \\
& \leq(m !)^{p-1} C^{m}\left(\frac{n}{(\log n)^{p}}\right)^{m}
\end{aligned}
$$

where the last step follows from Stirling's formula. This completes the proof of (2.15). 
By Hölder's inequality this shows that

$$
\begin{aligned}
& \left(\frac{(\log n)^{p}}{n}\right)^{m /(p-1)} \sup _{y_{1}, \cdots, y_{p}} \mathbb{E}^{\left(y_{1}, \cdots, y_{p}\right)}\left(J_{n}^{m /(p-1)}\right) \\
& \leq\left(\frac{(\log n)^{p}}{n}\right)^{m /(p-1)} \sup _{y_{1}, \cdots, y_{p}}\left\{\mathbb{E}^{\left(y_{1}, \cdots, y_{p}\right)}\left(J_{n}^{m}\right)\right\}^{1 /(p-1)} \\
& \leq\left(\frac{(\log n)^{p}}{n}\right)^{m /(p-1)}\left\{\mathbb{E}\left(J_{n}^{m}\right)\right\}^{1 /(p-1)} \leq C^{m} m !
\end{aligned}
$$

where the second inequality used [8], p.1053. Our theorem then follows from a Taylor expansion.

Remark. Theorem 2.2 is sharp in the sense that (2.12) does not hold if $\theta$ is too large. Indeed, by [21], for any $m=1,2, \cdots$,

$$
\frac{(\log n)^{p m}}{n^{m}} \mathbb{E} J_{n}^{m} \longrightarrow(2 \pi)^{p m} \operatorname{det}(\Gamma)^{m / 2} \mathbb{E} \alpha\left([0,1]^{p}\right)^{m}
$$

as $n \rightarrow \infty$, where $\alpha\left([0,1]^{p}\right)$ is the Brownian intersection local time formally defined by

$$
\alpha\left([0,1]^{p}\right)=\int_{\mathbb{R}^{d}}\left[\prod_{j=1}^{p} \int_{0}^{1} \delta_{x}\left(W_{j}(s)\right) d s\right] d x,
$$

and by Theorem 2.1 in [7]

$$
\mathbb{E} \exp \left\{\theta \alpha\left([0,1]^{p}\right)^{(p-1)^{-1}}\right\}=\infty
$$

for large $\theta$. The following theorem is sharp in the same sense.

Theorem 2.3 Assume that the planar random walk $S$ has finite second moments and zero mean. Then there exists $\theta>0$ such that

$$
\sup _{n} \mathbb{E} \exp \left\{\theta \frac{(\log n)^{2}}{n}\left|\bar{R}_{n}\right|\right\}<\infty \text {. }
$$

Proof. We first consider the case where $n$ is replaced by $2^{n}$. Let

$$
N=\left[2(\log 2)^{-1} \log n\right]
$$


so that $2^{N} \sim n^{2}$ and note that

$$
\begin{aligned}
& \text { 18) } \#\left\{S\left[1,2^{n}\right]\right\}=\sum_{k=1}^{2^{N}} \#\left\{S\left((k-1) 2^{n-N}, k 2^{n-N}\right]\right\} \\
& -\sum_{j=1}^{N} \sum_{k=1}^{2^{j-1}} \#\left\{S\left((2 k-2) 2^{n-j},(2 k-1) 2^{n-j}\right] \cap S\left((2 k-1) 2^{n-j},(2 k) 2^{n-j}\right]\right\} .
\end{aligned}
$$

Setting

$$
\beta_{k}=\#\left\{S\left((k-1) 2^{n-N}, k 2^{n-N}\right]\right\}
$$

and

$$
\alpha_{j, k}=\#\left\{S\left((2 k-2) 2^{n-j},(2 k-1) 2^{n-j}\right] \cap S\left((2 k-1) 2^{n-j},(2 k) 2^{n-j}\right]\right\}
$$

leads to the decomposition

$$
\bar{R}_{2^{n}}=\sum_{k=1}^{2^{N}} \bar{\beta}_{k}-\sum_{j=1}^{N} \sum_{k=1}^{2^{j-1}} \bar{\alpha}_{j, k}
$$

Recall that (Lemma 3 in 8 ),

$$
\sup _{n} \mathbb{E} \exp \left\{\lambda \frac{\log n}{n} \#\{S[1, n]\}\right\}<\infty
$$

for all $\lambda>0$. In particular,

$$
\sup _{n} \mathbb{E} \exp \left\{\lambda \frac{\log 2^{n-N}}{2^{n-N}}\left|\bar{\beta}_{1}\right|\right\}<\infty .
$$

Notice that $\bar{\beta}_{1}, \cdots, \bar{\beta}_{2^{N}}$ is an i.i.d. sequence with $\mathbb{E} \bar{\beta}_{1}=0$. By Lemma 1 in [3], there is a $\theta>0$ such that

$$
\sup _{n} \mathbb{E} \exp \left\{\theta 2^{-N / 2} \frac{\log 2^{n-N}}{2^{n-N}}\left|\sum_{k=1}^{2^{N}} \bar{\beta}_{k}\right|\right\}<\infty .
$$

By the choice of $N$ one can see that there is a $c>0$ independent of $n$ such that

$$
2^{-N / 2} \frac{\log 2^{n-N}}{2^{n-N}} \geq c \frac{\left(\log 2^{n}\right)^{2}}{2^{n}}
$$


So there is some $\theta>0$ such that

$$
\sup _{n} \mathbb{E} \exp \left\{\theta \frac{\left(\log 2^{n}\right)^{2}}{2^{n}}\left|\sum_{k=1}^{2^{N}} \bar{\beta}_{k}\right|\right\}<\infty .
$$

We need to show that for some $\theta>0$,

$$
\sup _{n} \mathbb{E} \exp \left\{\theta \frac{\left(\log 2^{n}\right)^{2}}{2^{n}}\left|\sum_{j=1}^{N} \sum_{k=1}^{2^{j-1}} \bar{\alpha}_{j, k}\right|\right\}<\infty
$$

Set

$$
\tilde{J}_{n}=\#\left\{S[1, n] \cap S^{\prime}[1, n]\right\} \quad n=1,2, \cdots,
$$

where $S^{\prime}$ is an independent copy of the random walk $S$. In our notation, for each $1 \leq j \leq N,\left\{\bar{\alpha}_{j, 1}, \cdots, \bar{\alpha}_{j, 2^{j-1}}\right\}$ is an i.i.d. sequence with the same distribution as $\tilde{J}_{2^{n-j}}$. By Theorem 2.2 (with $p=2$ ), there is a $\delta>0$ such that

$$
\sup _{n} \sup _{j \leq N} \mathbb{E} \exp \left\{\delta \frac{\left(\log 2^{n-j}\right)^{2}}{2^{n-j}}\left|\bar{\alpha}_{j, 1}\right|\right\}<\infty .
$$

By Lemma 1 in [3] again, there is a $\bar{\theta}>0$ such that

$$
\sup _{n} \sup _{j \leq N} \mathbb{E} \exp \left\{\bar{\theta} 2^{-j / 2} \frac{\left(\log 2^{n}\right)^{2}}{2^{n-j}}\left|\sum_{k=1}^{2^{j-1}} \bar{\alpha}_{j, k}\right|\right\}<\infty .
$$

Hence for some $\theta>0$

$$
C(\theta) \equiv \sup _{n} \sup _{j \leq N} \mathbb{E} \exp \left\{\theta 2^{j / 2} \frac{\left(\log 2^{n}\right)^{2}}{2^{n}}\left|\sum_{k=1}^{2^{j-1}} \bar{\alpha}_{j, k}\right|\right\}<\infty .
$$

Write

$$
\lambda_{N}=\prod_{j=1}^{N}\left(1-2^{-j / 2}\right) \text { and } \lambda_{\infty}=\prod_{j=1}^{\infty}\left(1-2^{-j / 2}\right) .
$$


Using Hölder's inequality with $1 / p=1-2^{-N / 2}, 1 / q=2^{-N / 2}$ we have

$$
\begin{aligned}
\mathbb{E} \exp \left\{\lambda_{N} \theta\right. & \left.\frac{\left(\log 2^{n}\right)^{2}}{2^{n}}\left|\sum_{j=1}^{N} \sum_{k=1}^{2^{j-1}} \bar{\alpha}_{j, k}\right|\right\} \\
\leq & \left(\mathbb{E} \exp \left\{\lambda_{N-1} \theta \frac{\left(\log 2^{n}\right)^{2}}{2^{n}}\left|\sum_{j=1}^{N-1} \sum_{k=1}^{2^{j-1}} \bar{\alpha}_{j, k}\right|\right\}\right)^{1-2^{-N / 2}} \\
& \times\left(\mathbb{E} \exp \left\{\lambda_{N} \theta 2^{N / 2} \frac{\left(\log 2^{n}\right)^{2}}{2^{n}}\left|\sum_{k=1}^{2^{N-1}} \bar{\alpha}_{N, k}\right|\right\}\right)^{2^{-N / 2}} \\
\leq & \mathbb{E} \exp \left\{\lambda_{N-1} \theta \frac{\left(\log 2^{n}\right)^{2}}{2^{n}}\left|\sum_{j=1}^{N-1} \sum_{k=1}^{2^{j-1}} \bar{\alpha}_{j, k}\right|\right\} \cdot C(\theta)^{2^{-N / 2}}
\end{aligned}
$$

since $\lambda_{N}<1$. Repeating this procedure,

$$
\begin{aligned}
& \mathbb{E} \exp \{\left.\lambda_{N} \theta \frac{\left(\log 2^{n}\right)^{2}}{2^{n}}\left|\sum_{j=1}^{N} \sum_{k=1}^{2^{j-1}} \bar{\alpha}_{j, k}\right|\right\} \\
& \leq C(\theta)^{2^{-1 / 2}+\cdots+2^{-N / 2}} \leq C(\theta)^{2^{-1 / 2}\left(1-2^{-1 / 2}\right)^{-1}}
\end{aligned}
$$

So we have

$$
\sup _{n} \mathbb{E} \exp \left\{\lambda_{\infty} \theta \frac{\left(\log 2^{n}\right)^{2}}{2^{n}}\left|\sum_{j=1}^{N} \sum_{k=1}^{2^{j-1}} \bar{\alpha}_{j, k}\right|\right\} \leq C(\theta)^{2^{-1 / 2}\left(1-2^{-1 / 2}\right)^{-1}} .
$$

We have proved (2.20) and therefore (2.17) when $n$ is the power of 2 . We now prove Theorem 2.3 for general $n$. Given an integer $n \geq 2$, we have the following unique representation:

$$
n=2^{m_{1}}+2^{m_{2}}+\cdots+2^{m_{l}}
$$

where $m_{1}>m_{2}>\cdots m_{l} \geq 0$ are integers. Write

$$
n_{0}=0 \text { and } n_{i}=2^{m_{1}}+\cdots+2^{m_{i}} \quad i=1, \cdots, l .
$$

Then

$$
\begin{aligned}
\#\{S[1, n]\} & =\sum_{i=1}^{l} \#\left\{S\left(n_{i-1}, n_{i}\right]\right\}-\sum_{i=1}^{l-1} \#\left\{S\left(n_{i-1}, n_{i}\right] \cap S\left(n_{i}, n\right]\right\} \\
& =\sum_{i=1}^{l} B_{i}-\sum_{i=1}^{l-1} A_{i} .
\end{aligned}
$$


Write

$$
\sum_{i=1}^{l} B_{i}=\sum_{i}^{\prime} B_{i}+\sum_{i}^{\prime \prime} B_{i}
$$

where $\sum_{i}^{\prime}$ is the summation over $i$ with $2^{m_{i}} \geq \sqrt{n}$ and $\sum_{i}{ }^{\prime \prime}$ is the summation over $i$ with $2^{m_{i}}<\sqrt{n}$. We also define the products $\prod_{i}^{\prime}$ and $\prod_{i}{ }^{\prime \prime}$ in a similar manner. Then

$$
\begin{aligned}
\mathbb{E} \exp \{\theta & \left.\frac{(\log n)^{2}}{n}\left|\sum_{i}^{\prime}\left(B_{i}-\mathbb{E} B_{i}\right)\right|\right\} \\
& \leq \prod_{i}^{\prime}\left(\mathbb{E} \exp \left\{\theta \frac{(\log n)^{2}}{n} 2^{-m_{i}}\left(\sum_{j}^{\prime} 2^{m_{j}}\right)\left|\bar{R}_{2^{m_{i}}}\right|\right\}\right)^{2^{m_{i}}\left(\sum_{j}^{\prime} 2^{m_{j}}\right)^{-1}} \\
& \leq \prod_{i}^{\prime}\left(\mathbb{E} \exp \left\{4 \theta \frac{\left(\log 2^{m_{i}}\right)^{2}}{2^{m_{i}}}\left|\bar{R}_{2^{m_{i}}}\right|\right\}\right)^{2^{m_{i}}\left(\sum_{j}^{\prime} 2^{m_{j}}\right)^{-1}} \\
& \leq \sup _{m} \mathbb{E} \exp \left\{4 \theta \frac{\left(\log 2^{m}\right)^{2}}{2^{m}}\left|\bar{R}_{2^{m}}\right|\right\}
\end{aligned}
$$

Assume that the set $\left\{1 \leq i \leq l ; 2^{m_{i}}<\sqrt{n}\right\}$ is non-empty. We have

$$
\sum_{i}^{\prime \prime} 2^{m_{i}} \leq 2 \sqrt{n}
$$

So we have

$$
\frac{(\log n)^{2}}{n} \leq \frac{1}{\sqrt{n}} \leq 2\left(\sum_{i}^{\prime \prime} 2^{m_{i}}\right)^{-1}
$$


Hence

$$
\begin{aligned}
\mathbb{E} \exp \{\theta & \left.\frac{(\log n)^{2}}{n}\left|\sum_{i}^{\prime \prime}\left(B_{i}-\mathbb{E} B_{i}\right)\right|\right\} \\
& \leq \prod_{i}^{\prime \prime}\left(\mathbb{E} \exp \left\{\theta \frac{(\log n)^{2}}{n} 2^{-m_{i}}\left(\sum_{j}^{\prime \prime} 2^{m_{j}}\right)\left|\bar{R}_{2^{m_{i}}}\right|\right\}\right)^{2^{m_{i}}\left(\sum_{j}^{\prime \prime} 2^{m_{j}}\right)^{-1}} \\
& \leq \prod_{i}^{\prime \prime}\left(\mathbb{E} \exp \left\{2 \theta \frac{1}{2^{m_{i}}}\left|\bar{R}_{2^{m_{i}}}\right|\right\}\right)^{2^{m_{i}}\left(\sum_{j}{ }^{\prime \prime} 2^{m_{j}}\right)^{-1}} \\
& \leq \sup _{m} \mathbb{E} \exp \left\{2 \theta \frac{1}{2^{m}}\left|\bar{R}_{2^{m}}\right|\right\} .
\end{aligned}
$$

By the Cauchy-Schwarz inequality and what we have proved in the previous step, there exists $\theta>0$ such that

$$
\mathbb{E} \exp \left\{\theta \frac{(\log n)^{2}}{n}\left|\sum_{i=1}^{l}\left(B_{i}-\mathbb{E} B_{i}\right)\right|\right\}
$$

is bounded uniformly in $n$. By the fact that

$$
n-n_{i}=2^{m_{i+1}}+\cdots+2^{m_{l}} \leq 2^{m_{i}}
$$

we have

$$
A_{i} \stackrel{d}{=} \#\left\{S\left[1,2^{m_{i}}\right] \cap S^{\prime}\left[1, n-n_{i}\right]\right\} \leq J_{2^{m_{i}}}
$$

By (2.14) there is a constant $C>0$ independent of $n$ such that

$$
\begin{aligned}
\sum_{i=1}^{l-1} \mathbb{E} A_{i} & \leq \sum_{i=1}^{l-1} \mathbb{E} J_{2^{m_{i}}} \leq C \sum_{i=1}^{l} \frac{2^{m_{i}}}{m_{i}^{2}} \\
& \leq C \sum_{m_{i}<l / 2} \frac{2^{m_{i}}}{m_{i}^{2}}+C \sum_{m_{i} \geq l / 2}^{l} \frac{2^{m_{i}}}{m_{i}^{2}} \\
& \leq C 2^{l / 2}+C \frac{n}{(\log n)^{2}} \\
& \leq C \frac{n}{(\log n)^{2}}
\end{aligned}
$$


It remains to show that

$$
\sup _{n} \mathbb{E} \exp \left\{\theta \frac{(\log n)^{2}}{n} \sum_{i=1}^{l-1} A_{i}\right\}<\infty .
$$

Using (2.24) this follows from (2.12), (with $p=2$ ), and the same argument used for $B_{1}-\mathbb{E} B_{1}, \cdots, B_{l}-\mathbb{E} B_{l}$.

In view of the remark prior to Theorem 2.3 the next result shows that $\bar{R}_{n}$ has a non-symmetric tail behavior.

Theorem 2.4 Under the assumptions of Theorem 2.3,

$$
\sup _{n} \mathbb{E} \exp \left\{\theta \frac{(\log n)^{2}}{n} \bar{R}_{n}\right\}<\infty
$$

for all $\theta>0$.

Proof. By Theorem 2.3, (2.26) holds for some $\theta_{0}>0$. For $\theta>\theta_{0}$, take an integer $m \geq 1$ such that $m^{-1} \theta<\theta_{0}$. It is easy to see that it suffices to prove

$$
\sup _{n} \mathbb{E} \exp \left\{\theta \frac{(\log n)^{2}}{m n} \bar{R}_{n m}\right\}<\infty \text {. }
$$

Set $\zeta_{j n}=\#\{S((j-1) n, j n]\}$. By the facts that

$$
\bar{R}_{n m} \leq \sum_{j=1}^{m} \overline{\zeta_{j n}}+\left(\sum_{j=1}^{m} \mathbb{E} \zeta_{j n}\right)-\mathbb{E} R_{n m}
$$

and that by (2.2) and (2.3),

$$
\begin{aligned}
\left(\sum_{j=1}^{m} \mathbb{E} \zeta_{j n}\right)-\mathbb{E} R_{m n} & =m \mathbb{E} R_{n}-\mathbb{E} R_{m n} \\
& =\frac{m n}{\mathcal{H}(n)}+O\left(\frac{m n}{\mathcal{H}(n)^{2}}\right)-\frac{m n}{\mathcal{H}(m n)}+O\left(\frac{m n}{\mathcal{H}(m n)^{2}}\right) \\
& =\frac{m n}{\mathcal{H}(n) \mathcal{H}(m n)}\left((\mathcal{H}(m n)-\mathcal{H}(n))+O\left(\frac{n}{\log ^{2} n}\right)\right. \\
& =O\left(\frac{n}{(\log n)^{2}}\right)
\end{aligned}
$$


as $n \rightarrow \infty$ (note $m$ is fixed), there is a constant $C_{m, \theta}>0$ depending only on $m$ and $\theta$ such that

$$
\mathbb{E} \exp \left\{\theta \frac{(\log n)^{2}}{m n} \bar{R}_{n m}\right\} \leq C_{m}\left(\mathbb{E} \exp \left\{\theta \frac{(\log n)^{2}}{m n} \bar{R}_{n}\right\}\right)^{m} .
$$

So we have (2.27).

\section{Moderate deviations for $R_{n}-\mathbb{E} R_{n}$}

We can now prove Theorem 1.1

Proof. We first prove the upper bound. Let $t>0$ and write $K=\left[t^{-1} b_{n}\right]$. Divide $[1, n]$ into $K$ disjoint subintervals, each of length $[n / K]$ or $[n / K]+1$. Call the $i^{\text {th }}$ subinterval $I_{i}$. Let $E_{i}=\#\left\{S\left(I_{i}\right)\right\}$. Then

$$
\bar{R}_{n} \leq \sum_{j=1}^{K} \bar{E}_{j}+\left(\sum_{j=1}^{K} \mathbb{E} E_{j}\right)-\mathbb{E} R_{n}
$$

From (2.1) we have

$$
\begin{array}{rl}
\sum_{j=1}^{K} & \mathbb{E} E_{j}-\mathbb{E} R_{n} \\
= & K \frac{n / K}{\mathcal{H}([n / K])}-\frac{n}{\mathcal{H}(n)}+\frac{1}{2 \pi \sqrt{\operatorname{det} \Gamma}}\left\{K \frac{n / K}{\mathcal{H}^{2}([n / K])}-\frac{n}{\mathcal{H}^{2}(n)}\right\}+o\left(\frac{n}{\mathcal{H}^{2}(n)}\right) \\
= & \frac{n(\mathcal{H}(n)-\mathcal{H}([n / K]))}{\mathcal{H}^{2}(n)}\left\{1+\frac{\mathcal{H}(n)-\mathcal{H}([n / K])}{\mathcal{H}([n / K])}\right\} \\
& +\frac{n}{\mathcal{H}^{2}(n)}\left\{\frac{\mathcal{H}^{2}(n)-\mathcal{H}^{2}([n / K])}{\mathcal{H}^{2}([n / K])}\right\}+o\left(\frac{n}{\mathcal{H}^{2}(n)}\right),
\end{array}
$$

where the error term can be taken to be independent of $\left\{b_{n}\right\}$. (This is where the hypothesis $\log b_{n}=o\left((\log n)^{1 / 2}\right)$ is used.) Since

$$
\mathcal{H}(n)-\mathcal{H}([n / K])=\sum_{k=[n / K]+1}^{n} \mathbb{P}\left\{S_{k}=0\right\} \sim \frac{\log K}{2 \pi \sqrt{\operatorname{det} \Gamma}},
$$


we have

$$
\sum_{j=1}^{K} \mathbb{E} E_{j}-\mathbb{E} R_{n}=\frac{n(\mathcal{H}(n)-\mathcal{H}([n / K]))}{\mathcal{H}^{2}(n)}+o\left(\frac{n}{\mathcal{H}^{2}(n)}\right)
$$

Hence for any $\lambda>0$,

$$
\begin{aligned}
\mathbb{P}\left\{\bar{R}_{n}\right. & \left.\geq \frac{n}{\mathcal{H}^{2}(n)}\left(\mathcal{H}(n)-\mathcal{H}\left(\left[n / b_{n}\right]\right)\right)\right\} \\
& \leq \exp \left\{-\lambda b_{n}\left(\mathcal{H}(n)-\mathcal{H}\left(\left[n / b_{n}\right]\right)\right)\right\} \mathbb{E} \exp \left\{\lambda \frac{\mathcal{H}^{2}(n) b_{n}}{n} \bar{R}_{n}\right\} \\
& \leq \exp \left\{-\lambda b_{n}\left(\mathcal{H}([n / K])-\mathcal{H}\left(\left[n / b_{n}\right]\right)\right)+o\left(b_{n}\right)\right\}\left(\mathbb{E} \exp \left\{\lambda \frac{\mathcal{H}^{2}(n) b_{n}}{n} \bar{E}_{1}\right\}\right)^{K} .
\end{aligned}
$$

Notice that

$$
\lim _{n \rightarrow \infty}\left(\mathcal{H}([n / K])-\mathcal{H}\left(\left[n / b_{n}\right]\right)\right)=\frac{\log t}{2 \pi \sqrt{\operatorname{det} \Gamma}}
$$

and that by [21, Theorem 6.1],

$$
\frac{\mathcal{H}^{2}(n) b_{n}}{n} \bar{E}_{1} \stackrel{d}{\longrightarrow}-\frac{2 \pi t}{2 \pi \sqrt{\operatorname{det} \Gamma}} \gamma_{1}
$$

where $\gamma_{t}$ is the renormalized self-intersection local time of a planar Brownian motion. By Theorem 2.4 and the dominated convergence theorem,

$$
\mathbb{E} \exp \left\{\lambda \frac{\mathcal{H}^{2}(n) b_{n}}{n} \bar{E}_{1}\right\} \longrightarrow \mathbb{E} \exp \left\{-\lambda \frac{2 \pi t}{2 \pi \sqrt{\operatorname{det} \Gamma}} \gamma_{1}\right\}
$$

Consequently,

$$
\begin{aligned}
\limsup _{n \rightarrow \infty} & b_{n}^{-1} \log \mathbb{P}\left\{\bar{R}_{n} \geq \frac{n}{\mathcal{H}^{2}(n)}\left(\mathcal{H}(n)-\mathcal{H}\left(\left[n / b_{n}\right]\right)\right)\right\} \\
\leq & -\lambda \frac{\log t}{2 \pi \sqrt{\operatorname{det} \Gamma}}+\frac{1}{t} \log \mathbb{E} \exp \left\{-\lambda \frac{2 \pi t}{2 \pi \sqrt{\operatorname{det} \Gamma}} \gamma_{1}\right\} \\
= & \frac{\lambda}{2 \pi \sqrt{\operatorname{det} \Gamma}} \log \frac{\lambda}{2 \pi \sqrt{\operatorname{det} \Gamma}} \\
& +\frac{1}{t} \log \mathbb{E} \exp \left\{-\frac{\lambda t}{2 \pi \sqrt{\operatorname{det} \Gamma}} \log \frac{\lambda t}{2 \pi \sqrt{\operatorname{det} \Gamma}}-\lambda \frac{2 \pi t}{2 \pi \sqrt{\operatorname{det} \Gamma}} \gamma_{1}\right\} .
\end{aligned}
$$


By [2], see the proof of Theorem 3.2, the limit

$$
C \equiv \lim _{t \rightarrow \infty} \frac{1}{t} \log \mathbb{E} \exp \left\{-t \log t-2 \pi t \gamma_{1}\right\}
$$

exists. Set

$$
L=\exp (-1-C)
$$

Letting $t \rightarrow \infty$ in (3.3) gives

$$
\begin{gathered}
\limsup _{n \rightarrow \infty} b_{n}^{-1} \log \mathbb{P}\left\{\bar{R}_{n} \geq \frac{n}{\mathcal{H}^{2}(n)}\left(\mathcal{H}(n)-\mathcal{H}\left(\left[n / b_{n}\right]\right)\right)\right\} \\
\leq \frac{\lambda}{2 \pi \sqrt{\operatorname{det} \Gamma}} \log \frac{\lambda}{2 \pi \sqrt{\operatorname{det} \Gamma}}+C \frac{\lambda}{2 \pi \sqrt{\operatorname{det} \Gamma}}
\end{gathered}
$$

Taking

$$
\frac{\lambda}{2 \pi \sqrt{\operatorname{det} \Gamma}}=\exp \{-1-C\}
$$

then yields

$$
\begin{gathered}
\limsup _{n \rightarrow \infty} b_{n}^{-1} \log \mathbb{P}\left\{\bar{R}_{n} \geq \frac{n}{\mathcal{H}^{2}(n)}\left(\mathcal{H}(n)-\mathcal{H}\left(\left[n / b_{n}\right]\right)\right)\right\} \\
\leq-\exp \{-1-C\}=-L
\end{gathered}
$$

We now prove the lower bound. The proof is similar to that of Proposition 4.4 of [6]. Fix $n$ and let $K=\left[b_{n}\right]$. Let $M=\left[n / b_{n}\right]$. Let $I_{j}$ be the interval $\left(m_{j}, m_{j+1}\right]$, where the $m_{j}$ are integers such that $m_{0}=0, m_{K}=n$, and $m_{j+1}-m_{j}$ is equal to either $M$ or $M+1$.

Let $\mathbf{e}$ be a vector of length $\sqrt{M}$ and let $B(x, r)$ be the ball of radius $r$ about $x$. Set

$$
E_{j}=\#\left\{S\left(I_{j}\right)\right\}, \quad H_{j}=\#\left\{S\left(I_{j}\right) \cap S\left(I_{j-1}\right)\right\} .
$$

Let

$$
A_{j}=\left\{S_{m_{j+1}} \in B\left((j+1) \mathbf{e}, \frac{1}{8} \sqrt{M}\right)\right\} \cap\left\{S\left(I_{j}\right) \subset B\left(\left(j+\frac{1}{2}\right) \mathbf{e}, \sqrt{M}\right)\right\}
$$

and

$$
B_{j}=\left\{\bar{E}_{j} \log ^{2} M / M \geq-c_{1}\right\}
$$


where we will select $c_{1}$ in a moment. By the central limit theorem, we know $\mathbb{P}^{S_{m_{j-1}}}\left(A_{j}\right) \geq c_{2}$ on the event $A_{j-1}$ if $n$ is large. By [21, Theorem 6.1], $\mathbb{P}^{S_{m_{j-1}}}\left(A_{j} \cap B_{j}\right)>c_{2} / 2$ on the event $A_{j-1}$ if we take $c_{1}$ sufficiently large. If we let

$$
F=\bigcap_{j=0}^{K-1}\left(A_{j} \cap B_{j}\right)
$$

then by the Markov property applied $K-1$ times we have

$$
\mathbb{P}(F) \geq\left(c_{2} / 2\right)^{K-1} .
$$

On the set $F$ we have that $S\left(I_{j}\right)$ is disjoint from $S\left(I_{i}\right)$ if $|i-j|>1$, and so on $F$

$$
\bar{R}_{n}=\sum_{j=1}^{K} \bar{E}_{j}+\left(\left(\sum_{j=1}^{K} \mathbb{E} E_{j}\right)-\mathbb{E} R_{n}\right)-\sum_{j=1}^{K} H_{j} .
$$

On the set $F$ the event $B_{j}$ holds for each $j$, and so

$$
\sum_{j=1}^{K} \bar{E}_{j} \geq-\frac{c_{1} K M}{\log ^{2} M} \geq-\frac{c_{3} n}{\log ^{2} n} .
$$

As in (3.2),

$$
\left(\sum_{j=1}^{K} \mathbb{E} E_{j}\right)-\mathbb{E} R_{n}=\frac{n(\mathcal{H}(n)-\mathcal{H}([n / K]))}{\mathcal{H}(n)^{2}}+o\left(\frac{n}{\mathcal{H}(n)^{2}}\right)
$$

if $n$ is large.

Let $\Lambda>0$ be chosen in a moment. Let

$$
C_{1}=\left\{\sum_{\{j \text { odd }\}} H_{j} \geq \frac{n \Lambda}{\log ^{2} n}\right\}, \quad C_{2}=\left\{\sum_{\{j \text { even }\}} H_{j} \geq \frac{n \Lambda}{\log ^{2} n}\right\} .
$$

Set $G=F \cap C_{1}^{c} \cap C_{2}^{c}$. For $j$ odd the $H_{j}$ are independent, and by Lemma 4.6 of [6],

$$
\begin{aligned}
\mathbb{P}\left(C_{1}\right) & =\mathbb{P}\left(\sum_{\{j \text { odd }\}} \frac{H_{j}}{M / \log ^{2} M} \geq c_{4} K \Lambda\right) \\
& \leq e^{-c_{4} c_{5} K \Lambda} \mathbb{E} e^{c_{5} H_{j} \log ^{2} M / M} \\
& \leq e^{-c_{4} c_{5} K \Lambda} c_{6}^{K},
\end{aligned}
$$


where $c_{4}, c_{5}, c_{6}$ do not depend on $\Lambda$ and without loss of generality we may assume $c_{6}>1$. Choose $\Lambda$ large so that $e^{-c_{4} c_{5} \Lambda} \leq c_{6}^{-2}$. When $n$ is large, $K$ will be large, and then $\mathbb{P}\left(C_{1}\right) \leq \mathbb{P}(F) / 3$. We have a similar estimate for $\mathbb{P}\left(C_{2}\right)$, so

$$
\mathbb{P}(G) \geq\left(c_{2} / 2\right)^{K-1} / 3 .
$$

Set $v_{n}=\mathcal{H}(n)-\mathcal{H}\left(\left[n / b_{n}\right]\right)$. On the event $G$

$$
\sum_{j=1}^{K} H_{j} \leq 2 \frac{n \Lambda}{\log ^{2} n},
$$

and so combining (3.9), (3.10), and (3.11), on the event $G$

$$
\bar{R}_{n} \geq\left(1-\frac{c_{7}}{v_{n}}\right) n v_{n} / \mathcal{H}(n)^{2}
$$

Therefore

$$
\mathbb{P}\left(\bar{R}_{n} \geq\left(1-\frac{c_{7}}{v_{n}}\right) n v_{n} / \mathcal{H}(n)^{2}\right) \geq c_{8} c_{9}^{b_{n}} .
$$

Define $b_{n}^{\prime}$ by $v_{n}^{\prime}=\mathcal{H}(n)-\mathcal{H}\left(\left[n / b_{n}^{\prime}\right]\right)=v_{n}+c_{7}$. If we apply (3.13) with $b_{n}$ replaced by $b_{n}^{\prime}$, we have

$$
\begin{aligned}
\mathbb{P}\left(\bar{R}_{n} \geq\right. & \left.n v_{n} / \mathcal{H}(n)^{2}\right) \\
\quad & =\mathbb{P}\left(\bar{R}_{n} \geq\left(1-\frac{c_{7}}{v_{n}^{\prime}}\right) n v_{n}^{\prime} / \mathcal{H}(n)^{2}\right) \\
& \geq c_{8} c_{9}^{b_{n}^{\prime}} .
\end{aligned}
$$

We now take the logarithms of both sides, divide by $b_{n}$, and use the fact that the ratio $b_{n} / b_{n}^{\prime}$ is bounded above and below by positive constants to obtain the lower bound.

Proof of Corollary [1.3; Assume first that $S_{n}$ is strongly aperiodic. We have by Proposition 2.1 that

$$
\mathbb{P}\left(S_{n}=0\right)=\frac{1}{2 \pi n \sqrt{\operatorname{det} \Gamma}}+O\left(\frac{1}{n(\log n)^{1 / 2}}\right) .
$$

Then, if $\gamma$ denotes Euler's constant

$$
\sum_{k=1}^{n} \frac{1}{k}=\log n+\gamma+O\left(\frac{1}{n}\right)
$$


and

$$
\sum_{k=3}^{n} \frac{1}{k(\log k)^{1 / 2}} \leq \int_{2}^{n} \frac{d x}{x(\log x)^{1 / 2}} \leq c_{1}(\log n)^{1 / 2}
$$

so that

$$
\begin{aligned}
\mathcal{H}(n) & =\sum_{k=0}^{n} \mathbb{P}^{0}\left(S_{k}=0\right)=1+\frac{1}{2 \pi \sqrt{\operatorname{det} \Gamma}} \sum_{k=1}^{n}\left(\frac{1}{k}+O\left(\frac{1}{k(\log k)^{1 / 2}}\right)\right) \\
& =\frac{1}{2 \pi \sqrt{\operatorname{det} \Gamma}}\left(\log n+\gamma+O\left((\log n)^{1 / 2}\right)\right) \\
& =\frac{\log n}{2 \pi \sqrt{\operatorname{det} \Gamma}}\left(1+O\left(\frac{1}{(\log n)^{1 / 2}}\right)\right) .
\end{aligned}
$$

Similarly we

$$
\sum_{k=\left[n / b_{n}\right]+1}^{n} \frac{1}{k(\log k)^{1 / 2}} \leq c_{2}\left((\log n)^{1 / 2}-\left(\log \left(n / b_{n}\right)\right)^{1 / 2}\right)
$$

To evaluate this note that

$$
\begin{aligned}
\left(\log \left(n / b_{n}\right)\right)^{1 / 2} & =\left(\log n-\log b_{n}\right)^{1 / 2} \\
& =(\log n)^{1 / 2}\left(1-\log b_{n} / \log n\right)^{1 / 2} \\
& =(\log n)^{1 / 2}\left(1+O\left(\log b_{n} / \log n\right)\right) \\
& =(\log n)^{1 / 2}+O\left(\log b_{n} /(\log n)^{1 / 2}\right)
\end{aligned}
$$

by our assumption that $\log b_{n}=o\left((\log n)^{1 / 2}\right)$. It follows that

$$
\begin{aligned}
\mathcal{H}(n)-\mathcal{H}\left(\left[n / b_{n}\right]\right) & =\frac{1}{2 \pi \sqrt{\operatorname{det} \Gamma}} \sum_{k=\left[n / b_{n}\right]+1}^{n}\left(\frac{1}{k}+O\left(\frac{1}{k(\log k)^{1 / 2}}\right)\right) \\
& =\frac{1}{2 \pi \sqrt{\operatorname{det} \Gamma}}\left(\log b_{n}+O\left(\frac{\log b_{n}}{(\log n)^{1 / 2}}\right)\right) \\
& =\frac{\log b_{n}}{2 \pi \sqrt{\operatorname{det} \Gamma}}\left(1+O\left(\frac{1}{(\log n)^{1 / 2}}\right)\right) .
\end{aligned}
$$


We then have that

$$
\begin{aligned}
\frac{n}{\mathcal{H}(n)^{2}} & \left(\mathcal{H}(n)-\mathcal{H}\left(\left[n / b_{n}\right]\right)\right) \\
& =2 \pi \sqrt{\operatorname{det} \Gamma} \frac{n \log b_{n}}{(\log n)^{2}}\left(1+O\left(\frac{1}{(\log n)^{1 / 2}}\right)\right) \\
& =2 \pi \sqrt{\operatorname{det} \Gamma} \frac{n \log b_{n}}{(\log n)^{2}}\left(1+a_{n}\right),
\end{aligned}
$$

where we use the last equality to define $a_{n}$. Let

$$
1+\widehat{a}_{n}=\left(1+a_{n}\right)^{-1}=1+O\left(\frac{1}{(\log n)^{1 / 2}}\right) .
$$

Then if we set

$$
\widehat{b}_{n}=: b_{n}^{1+\widehat{a}_{n}}=b_{n}^{\left(1+a_{n}\right)^{-1}}
$$

we see from (3.21) that

$$
\frac{n}{\mathcal{H}(n)^{2}}\left(\mathcal{H}(n)-\mathcal{H}\left(\left[n / \widehat{b}_{n}\right]\right)\right)=2 \pi \sqrt{\operatorname{det} \Gamma} \frac{n \log b_{n}}{(\log n)^{2}}
$$

Also, $\log \widehat{b}_{n}=\left(1+\widehat{a}_{n}\right) \log b_{n}=o\left((\log n)^{1 / 2}\right)$, so that Theorem 1.1 applies to $\widehat{b}_{n}$, and indeed to $\widehat{b}_{n}^{\theta}$ for any $\theta>0$.

Note that

$$
\begin{aligned}
\widehat{b}_{n}^{\theta}= & b_{n}\left(1+O\left(\frac{1}{(\log n)^{1 / 2}}\right)\right) \\
= & b_{n}^{\theta} \exp \left(O\left(\frac{\log b_{n}}{(\log n)^{1 / 2}}\right)\right) \\
= & b_{n}^{\theta}\left(1+o\left(1_{n}\right)\right)
\end{aligned}
$$

by our assumption that $\log b_{n}=o\left((\log n)^{1 / 2}\right)$. Hence by (3.24) and (3.25)

$$
\begin{aligned}
\widehat{b}_{n}^{-\theta} \log \mathbb{P} & \left\{\bar{R}_{n} \geq \frac{\theta n}{\mathcal{H}(n)^{2}}\left(\mathcal{H}(n)-\mathcal{H}\left(\left[n / \widehat{b}_{n}\right]\right)\right)\right\} \\
& =\left(1+o\left(1_{n}\right)\right) b_{n}^{-\theta} \log \mathbb{P}\left\{\bar{R}_{n} \geq 2 \pi \theta \sqrt{\operatorname{det} \Gamma} \frac{n \log b_{n}}{(\log n)^{2}}\right\}
\end{aligned}
$$


Together with Proposition 2.1 Theorem 1.1 applied to $\widehat{b}_{n}^{\theta}$ proves the corollary in the strongly aperiodic case. The modifications to handle the case where $S_{n}$ is not strongly aperiodic are very similar to those in Section 2 of [23].

\section{Moderate deviations for $\mathbb{E} R_{n}-R_{n}$}

To avoid difficulties connected with subdividing time intervals, it is more convenient to look at the continuous time analogue of $S_{n}$. We let $T_{1}, T_{2}, \ldots$ be i.i.d. exponential random variables with parameter 1 that are independent of the sequence $S_{n}$. Define $Z_{t}=S_{n}$ if $\sum_{i=1}^{n} T_{i} \leq t<\sum_{i=1}^{n+1} T_{i} . Z_{t}$ is a Lévy process that waits an exponential length of time, then jumps according to $X_{1}$, and then repeats the procedure. Define $N_{t}=n$ if $\sum_{i=1}^{n} T_{i} \leq t<\sum_{i=1}^{n+1} T_{i}$. Note that $N_{t}$ is a Poisson process with $\mathbb{E} N_{t}=t$ and that $Z_{t}=S_{N_{t}}$. We write $|Z[a, b]|$ for the cardinality of $\left\{Z_{s}: s \in[a, b]\right\}$.

Theorems 2.2 and 2.3 have the following analogues for continuous time processes. We omit the proofs, which are almost identical to the proofs given for the discrete time random walks.

Lemma 4.1 Let $Z_{1}(t), \cdots, Z_{p}(t)$ be independent copies of $Z(t)$. There is $C>0$ such that

$$
\sup _{y_{1}, \cdots, y_{p}} \mathbb{E}^{\left(y_{1}, \cdots, y_{p}\right)}\left|Z_{1}[0, t] \cap \cdots \cap Z_{p}[0, t]\right|^{m} \leq C^{m}(m !)^{p-1}\left(\frac{t}{(\log t)^{p}}\right)^{m} .
$$

Consequently, there is $\theta>0$ such that

$$
\sup _{t} \sup _{y_{1}, \cdots, y_{p}} \mathbb{E}^{\left(y_{1}, \cdots, y_{p}\right)} \exp \left\{\theta\left(\frac{(\log t)^{p}}{t}\left|Z_{1}[0, t] \cap \cdots \cap Z_{p}[0, t]\right|\right)^{(p-1)^{-1}}\right\}<\infty .
$$

Lemma 4.2 There is $\theta>0$ such that

$$
\sup _{t} \mathbb{E} \exp \left\{\theta \frac{(\log t)^{2}}{t}|\mathbb{E}| Z[0, t]|-| Z[0, t]||\right\}<\infty .
$$

Consequently

$$
\limsup _{t \rightarrow \infty} \frac{1}{b_{t}} \log \mathbb{P}\left\{|\mathbb{E}| Z[0, t]|-| Z[0, t]|| \geq \lambda \frac{t b_{t}}{(\log t)^{2}}\right\} \leq-\theta \lambda .
$$


We will prove Theorem 1.5 by first proving the following analogue for $Z_{t}$.

Theorem 4.3 For any $\lambda>0$ and for any $b_{t}$ satisfying $b_{t} \rightarrow \infty$ and $b_{t}=$ $o\left((\log t)^{1 / 5}\right)$ as $t \rightarrow \infty$, we have

$$
\begin{aligned}
& \lim _{t \rightarrow \infty} \frac{1}{b_{t}} \log \mathbb{P}\left\{|\mathbb{E}| Z[0, t]|-| Z[0, t]|| \geq \lambda \frac{t b_{t}}{(\log t)^{2}}\right\} \\
& =-(2 \pi)^{-2} \operatorname{det}(\Gamma)^{-1 / 2} \kappa(2,2)^{-4} \lambda .
\end{aligned}
$$

The next proposition shows that Theorem 1.5 follows from Theorem 4.3 and Theorem [1.1.

Proposition 4.4 For any $\varepsilon>0$,

$$
\lim _{n \rightarrow \infty} \frac{1}{b_{n}} \log \mathbb{P}\left\{|\overline{|Z[0, n]|}-\overline{|S[0, n]|}| \geq \varepsilon \frac{n b_{n}}{(\log n)^{2}}\right\}=-\infty
$$

Remark 4.5 Our proof actually gives a stronger result, but this is all we need.

Proof. Observe that if $n>m$, then

$$
|\mathbb{E}| S[0, n]|-\mathbb{E}| S[0, m]|| \leq \mathbb{E}|S[m, n]|=\mathbb{E}|S[0, n-m]| \leq n-m .
$$

Consequently,

$$
\begin{aligned}
|\mathbb{E}| Z[0, n]|-\mathbb{E}| S[0, n]|| & =|\mathbb{E}| S\left[0, N_{n}\right]|-\mathbb{E}| S[0, n]|| \\
& \leq \mathbb{E}\left|N_{n}-n\right| \leq C \sqrt{n}
\end{aligned}
$$

Hence, it suffices to show that for any $\varepsilon>0$

$$
\lim _{n \rightarrow \infty} \frac{1}{b_{n}} \log \mathbb{P}\left\{|| Z[0, n]|-| S[0, n]|| \geq \varepsilon \frac{\sqrt{n} b_{n}^{3 / 2}}{\log n}\right\}=-\infty .
$$


Let $M>0$ be fixed. On the event $\left\{\left|N_{n}-n\right| \leq M \sqrt{n b_{n}}\right\}$

$$
\begin{aligned}
|| Z[0, n] \mid & -|S[0, n]||\leq| S\left[N_{n} \wedge n, N_{n} \vee n\right] \mid \\
& \stackrel{d}{=}\left|S\left[0, N_{n} \vee n-N_{n} \wedge n\right]\right| \leq\left|S\left[0,2 M \sqrt{n b_{n}}\right]\right|
\end{aligned}
$$

So we have

$$
\begin{aligned}
& \mathbb{P}\left\{|| Z[0, n]|-| S[0, n]|| \geq \varepsilon \frac{\sqrt{n} b_{n}^{3 / 2}}{\log n}\right\} \\
& \quad \leq \mathbb{P}\left\{\left|S\left[0,2 M \sqrt{n b_{n}}\right]\right| \geq \varepsilon \frac{\sqrt{n} b_{n}^{3 / 2}}{\log n}\right\}+\mathbb{P}\left\{\left|N_{n}-n\right| \geq M \sqrt{n b_{n}}\right\} .
\end{aligned}
$$

By Lemma 3 in [8],

$$
\sup _{n} \mathbb{E} \exp \left\{\theta \frac{\log n}{\sqrt{n b_{n}}} S\left[0,2 M \sqrt{n b_{n}}\right]\right\}<\infty, \quad \theta>0
$$

By the Chebyshev inequality one can see that

$$
\lim _{n \rightarrow \infty} \frac{1}{b_{n}} \log \mathbb{P}\left\{\left|S\left[0,2 M \sqrt{n b_{n}}\right]\right| \geq \varepsilon \frac{\sqrt{n} b_{n}^{3 / 2}}{\log n}\right\}=-\infty .
$$

By the classical moderate deviation principle ([13, Theorem 3.7.1]),

$$
\lim _{n \rightarrow \infty} \frac{1}{b_{n}} \log \mathbb{P}\left\{\left|N_{n}-n\right| \geq M \sqrt{n b_{n}}\right\}=-\frac{M^{2}}{2} .
$$

Thus,

$$
\limsup _{n \rightarrow \infty} \frac{1}{b_{n}} \log \mathbb{P}\left\{|| Z[0, n]|-| S[0, n]|| \geq \varepsilon \frac{\sqrt{n} b_{n}^{3 / 2}}{\log n}\right\} \leq-\frac{M^{2}}{2} .
$$

Letting $M \rightarrow \infty$ proves the proposition.

Thus we we need to prove Theorem 4.3. By the Gärtner-Ellis theorem ([13, Theorem 2.3.6]), to prove Theorem 4.3] it suffices to prove

$$
\begin{aligned}
& \lim _{t \rightarrow \infty} \frac{1}{b_{t}} \log \mathbb{E} \exp \left\{\theta \sqrt{\frac{b_{t}}{t}}(\log t)|\mathbb{E}| Z[0, t]|-| Z[0, t]||^{1 / 2}\right\} \\
& =(\theta \pi)^{2} \sqrt{\operatorname{det}(\Gamma)} \kappa(2,2)^{4} .
\end{aligned}
$$


Let $h(x)$ be a smooth symmetric probability density on $\mathbb{R}^{2}$ with compact support and write $h_{\varepsilon}(x)=\varepsilon^{-2} h\left(\varepsilon^{-1} x\right)$. We have

$$
\Lambda_{\varepsilon}(t) \equiv \sum_{x \in \mathbb{Z}^{2}} h_{\varepsilon}\left(\frac{x}{\sqrt{t}}\right) \sim t, \quad t \rightarrow \infty .
$$

The following lemma describing exponential asymptotics for the smoothed range will be proved in Section 5

\section{Lemma 4.6 Let}

$$
A_{t}(\varepsilon) \equiv \Lambda_{\varepsilon}\left(\frac{t}{b_{t}}\right)^{-2} \sum_{x \in \mathbb{Z}^{2}}\left[\sum_{y \in Z[0, t]} h_{\varepsilon}\left(\sqrt{\frac{b_{t}}{t}}(x-y)\right)\right]^{2} .
$$

For any $\theta>0$,

$$
\begin{aligned}
\lim _{t \rightarrow \infty} & \frac{1}{b_{t}} \log \mathbb{E} \exp \left\{\theta \sqrt{\frac{b_{t}}{t}}(\log t)\left|A_{t}(\varepsilon)\right|^{1 / 2}\right\} \\
= & \sup _{g \in \mathcal{F}}\left\{2 \pi \theta \sqrt{\operatorname{det}(\Gamma)}\left(\int_{\mathbb{R}^{2}}\left|\left(g^{2} * h_{\varepsilon}\right)(x)\right|^{2} d x\right)^{1 / 2}\right. \\
& -\frac{1}{2} \int_{\mathbb{R}^{2}}\left\langle\nabla g(x),\left.\Gamma \nabla g(x)\right|^{2} d x\right\} .
\end{aligned}
$$

where

$$
\mathcal{F}=\left\{g \in W^{1,2}\left(\mathbb{R}^{2}\right) ; \quad\|g\|_{2}=1\right\} .
$$

Furthermore, for any $N=0,1, \ldots$ and any $\epsilon>0$,

$$
\begin{aligned}
& \lim _{t \rightarrow \infty} \frac{1}{b_{t}} \log \mathbb{E} \exp \left\{\theta \sqrt{\frac{b_{t}}{t}}(\log t)\right. \\
& \times\left.\left(\Lambda_{\varepsilon}\left(\frac{t}{b_{t}}\right)^{-2} \sum_{x \in \mathbb{Z}^{2}}\left[\sum_{y \in Z\left[0,2^{-N} t\right]} h_{\varepsilon}\left(\sqrt{\frac{b_{t}}{t}}(x-y)\right)\right]^{2}\right)^{1 / 2}\right\} \\
& \leq 2^{-N+2} \pi^{2} \theta^{2} \sqrt{\operatorname{det}(\Gamma)} \kappa(2,2)^{4} .
\end{aligned}
$$

The following lemma on exponential approximation will be proved in Section [6] In this lemma $Z^{\prime}$ denotes an independent copy of $Z$. 
Lemma 4.7 Let

$$
\begin{aligned}
B_{t}^{(j)}(\varepsilon) & \equiv \Lambda_{\varepsilon}\left(\frac{t}{b_{t}}\right)^{-2} \\
& \times \sum_{x \in \mathbb{Z}^{2}}\left[\sum_{y \in Z\left[0,2^{-j} t\right]} h_{\varepsilon}\left(\sqrt{\frac{b_{t}}{t}}(x-y)\right)\right]\left[\sum_{y^{\prime} \in Z^{\prime}\left[0,2^{-j} t\right]} h_{\varepsilon}\left(\sqrt{\frac{b_{t}}{t}}\left(x-y^{\prime}\right)\right)\right] .
\end{aligned}
$$

Then for any $\theta>0$ and any $j=0,1, \ldots$,

$$
\limsup _{\varepsilon \rightarrow 0} \limsup _{t \rightarrow \infty} \frac{1}{b_{t}}
$$

$$
\log \mathbb{E} \exp \left\{\theta \sqrt{\frac{b_{t}}{t}}(\log t)|| Z\left[0,2^{-j} t\right] \cap Z^{\prime}\left[0,2^{-j} t\right]\left|-B_{t}^{(j)}(\varepsilon)\right|^{1 / 2}\right\}=0 .
$$

These lemmas will be the key to proving Theorem 4.3. Before proving this theorem, we present a simple lemma which will be used several times in the proof of Theorem 4.3 .

Lemma 4.8 Let $l \geq 2$ be a fixed integer and let $\left\{\xi_{1}(\rho) ; \rho>0\right\}, \cdots,\left\{\xi_{l}(\rho) ; \rho>\right.$ $0\}$ be $l$ independent non-negative stochastic processes.

(a) If there is a constant $C_{1}>0$ such that for any $1 \leq j \leq l$,

$$
\limsup _{\rho \rightarrow 0^{+}} \rho \log \mathbb{P}\left\{\xi_{j}(\rho) \geq \lambda\right\} \leq-C_{1} \lambda, \quad \lambda>0
$$

then

$$
\limsup _{\rho \rightarrow 0^{+}} \rho \log \mathbb{P}\left\{\xi_{1}(\rho)+\cdots+\xi_{l}(\rho) \geq \lambda\right\} \leq-C_{1} \lambda, \quad \lambda>0
$$

(b) If there is a constant $C_{2}>0$ such that for any $1 \leq j \leq l$,

$$
\limsup _{\rho \rightarrow 0^{+}} \rho \log \mathbb{E} \exp \left\{\rho^{-1} \theta \sqrt{\xi_{j}(\rho)}\right\} \leq C_{2} \theta^{2}, \quad \theta>0
$$

then

(4.26) $\quad \limsup _{\rho \rightarrow 0^{+}} \rho \log \mathbb{E} \exp \left\{\rho^{-1} \theta \sqrt{\xi_{1}(\rho)+\cdots+\xi_{l}(\rho)}\right\} \leq C_{2} \theta^{2}, \quad \theta>0$ 
Proof. . Clearly, part (a) needs only to be proved in the case $l=2$. Given $0<\delta<\lambda$, let $0=a_{0}<a_{1}<\cdots<a_{N}=\lambda$ be a partition of $[0, \lambda]$ such that $a_{k}-a_{k-1}<\delta$. Then

$$
\begin{aligned}
\mathbb{P}\left\{\xi_{1}(\rho)+\xi_{2}(\rho) \geq \lambda\right\} & \leq \sum_{k=1}^{N} \mathbb{P}\left\{\xi_{1}(\rho) \in\left[a_{k-1}, a_{k}\right]\right\} \mathbb{P}\left\{\xi_{2}(\rho) \geq \lambda-a_{k}\right\} \\
& \leq \sum_{k=1}^{N} \mathbb{P}\left\{\xi_{1}(\rho) \geq a_{k-1}\right\} \mathbb{P}\left\{\xi_{2}(\rho) \geq \lambda-a_{k}\right\} .
\end{aligned}
$$

Hence

$$
\begin{aligned}
& \limsup _{\rho \rightarrow 0^{+}} \rho \log \mathbb{P}\left\{\xi_{1}(\rho)+\xi_{2}(\rho) \geq \lambda\right\} \\
& \leq \max _{1 \leq k \leq N}\left\{-C_{1} a_{k-1}-C_{1}\left(\lambda-a_{k}\right)\right\} \leq-C_{1}(\lambda-\delta) .
\end{aligned}
$$

Letting $\delta \rightarrow 0^{+}$proves part (a).

We now prove part (b). By Chebyshev's inequality, for any $\lambda>0$

$$
\limsup _{\rho \rightarrow 0^{+}} \rho \log \mathbb{P}\left\{\xi_{j}(\rho) \geq \lambda\right\} \leq-\sup _{\theta>0}\left\{\theta \sqrt{\lambda}-C_{2} \theta^{2}\right\}=-\frac{\lambda}{4 C_{2}} .
$$

By part (a)

$$
\limsup _{\rho \rightarrow 0^{+}} \rho \log \mathbb{P}\left\{\xi_{1}(\rho)+\cdots+\xi_{l}(\rho) \geq \lambda\right\} \leq-\frac{\lambda}{4 C_{2}}, \quad \lambda>0 .
$$

In addition, by the triangle inequality and by independence,

$$
\mathbb{E} \exp \left\{\rho^{-1} \theta \sqrt{\xi_{1}(\rho)+\cdots+\xi_{l}(\rho)}\right\} \leq \prod_{j=1}^{l} \mathbb{E} \exp \left\{\rho^{-1} \theta \sqrt{\xi_{j}(\rho)}\right\} .
$$

So by assumption, for any $\theta>0$,

$$
\limsup _{\rho \rightarrow 0^{+}} \rho \log \mathbb{E} \exp \left\{\rho^{-1} \theta \sqrt{\xi_{1}(\rho)+\cdots+\xi_{l}(\rho)}\right\}<\infty .
$$

By [13, Lemma 4.3.6],

$$
\begin{aligned}
& \limsup _{\rho \rightarrow 0^{+}} \rho \log \mathbb{E} \exp \left\{\rho^{-1} \theta \sqrt{\xi_{1}(\rho)+\cdots+\xi_{l}(\rho)}\right\} \\
& \leq \sup _{\lambda>0}\left\{\theta \sqrt{\lambda}-\frac{\lambda}{4 C_{2}}\right\}=C_{2} \theta^{2} .
\end{aligned}
$$


Proof of Theorem 4.3: We begin with the decomposition

$$
\begin{aligned}
& |Z[0, t]|=\sum_{k=1}^{2^{N}}\left|Z\left[\frac{k-1}{2^{N}} t, \frac{k}{2^{N}} t\right]\right| \\
& \quad \quad-\sum_{j=1}^{N} \sum_{k=1}^{2^{j-1}}\left|Z\left[\frac{2 k-2}{2^{j}} t, \frac{2 k-1}{2^{j}} t\right] \cap Z\left[\frac{2 k-1}{2^{j}} t, \frac{2 k}{2^{j}} t\right]\right| \\
& =: I_{t}-J_{t} .
\end{aligned}
$$

We first establish the upper bound. Let $\varepsilon>0$ be fixed. Since

$$
E|Z[0, t]|-|Z[0, t]|=\left(\mathbb{E} I_{t}-I_{t}\right)+J_{t}-\mathbb{E} J_{t} \leq\left(\mathbb{E} I_{t}-I_{t}\right)+J_{t},
$$

it follows that

$$
\text { (4.36) } \begin{aligned}
\mathbb{P}\left\{|\mathbb{E}| Z[0, t]|-| Z[0, t]|| \geq \lambda t b_{t} /(\log t)^{2}\right\} \\
\quad \leq \mathbb{P}\left\{\left|\mathbb{E} I_{t}-I_{t}\right| \geq \varepsilon t b_{t} /(\log t)^{2}\right\}+\mathbb{P}\left\{J_{t} \geq(\lambda-\varepsilon) t b_{t} /(\log t)^{2}\right\} .
\end{aligned}
$$

Notice that

$$
\left|\mathbb{E} I_{t}-I_{t}\right| \leq \sum_{k=1}^{2^{N}}|\mathbb{E}| Z\left[\frac{k-1}{2^{N}} t, \frac{k}{2^{N}} t\right]|-| Z\left[\frac{k-1}{2^{N}} t, \frac{k}{2^{N}} t\right] \mid .
$$

Replacing $t$ by $2^{-N} t, \lambda$ by $2^{N} \lambda$ and $b_{t}$ by $\widetilde{b}_{t}=: b_{2^{N} t}$ in (4.4) we obtain (4.38)

$$
\limsup _{t \rightarrow \infty} \frac{1}{b_{t}} \log \mathbb{P}\left\{|\mathbb{E}| Z\left[0,2^{-N} t\right]|-| Z\left[0,2^{-N} t\right]|| \geq \lambda \frac{t b_{t}}{(\log t)^{2}}\right\} \leq-2^{N} C \lambda .
$$

Hence by Lemma 4.8 ,

$$
\limsup _{t \rightarrow \infty} \frac{1}{b_{t}} \log \mathbb{P}\left\{\left|\mathbb{E} I_{t}-I_{t}\right| \geq \frac{\varepsilon t b_{t}}{(\log t)^{2}}\right\} \leq-\varepsilon C 2^{N} .
$$

By the triangle inequality,

$$
\mathbb{P}\left\{J_{t} \geq \frac{(\lambda-\varepsilon) t b_{t}}{(\log t)^{2}}\right\} \leq \sum_{j=1}^{N} \mathbb{P}\left\{\sum_{k=1}^{2^{j-1}} \xi_{j, k} \geq 2^{-j} \frac{(\lambda-\varepsilon) t b_{t}}{(\log t)^{2}}\right\}
$$


where for each $1 \leq j \leq N$,

$$
\xi_{j, k}(t)=\left|Z\left[\frac{2 k-2}{2^{j}} t, \frac{2 k-1}{2^{j}} t\right] \cap Z\left[\frac{2 k-1}{2^{j}} t, \frac{2 k}{2^{j}} t\right]\right|, \quad k=1, \cdots, 2^{j-1},
$$

forms an i.i.d. sequence with the same distribution as

$$
\left|Z\left[0,2^{-j} t\right] \cap Z^{\prime}\left[0,2^{-j} t\right]\right|
$$

By Theorem 1 in [8] (with $2^{-j} t$ instead of $t$ ), for any $\lambda>0$,

$$
\begin{gathered}
\lim _{t \rightarrow \infty} \frac{1}{b_{t}} \log \mathbb{P}\left\{\left|Z\left[0,2^{-j} t\right] \cap Z^{\prime}\left[0,2^{-j} t\right]\right| \geq \frac{\lambda t b_{t}}{(\log t)^{2}}\right\} \\
=-2^{j}(2 \pi)^{-2} \operatorname{det}(\Gamma)^{-1 / 2} \kappa(2,2)^{-4} \lambda .
\end{gathered}
$$

Therefore, by Lemma 4.8 ,

$$
\lim _{t \rightarrow \infty} \frac{1}{b_{t}} \log \mathbb{P}\left\{\sum_{k=1}^{2^{j-1}} \xi_{j, k} \geq \frac{\lambda t b_{t}}{(\log t)^{2}}\right\}=-2^{j}(2 \pi)^{-2} \operatorname{det}(\Gamma)^{-1 / 2} \kappa(2,2)^{-4} \lambda
$$

In particular,

$$
\begin{aligned}
\lim _{t \rightarrow \infty} & \frac{1}{b_{t}} \log \mathbb{P}\left\{\sum_{k=1}^{2^{j-1}} \xi_{j, k} \geq 2^{-j} \frac{(\lambda-\varepsilon) t b_{t}}{(\log t)^{2}}\right\} \\
& =-(2 \pi)^{-2} \operatorname{det}(\Gamma)^{-1 / 2} \kappa(2,2)^{-4}(\lambda-\varepsilon)
\end{aligned}
$$

and therefore by (4.40)

$$
\lim _{t \rightarrow \infty} \frac{1}{b_{t}} \log \mathbb{P}\left\{J_{t} \geq \frac{(\lambda-\varepsilon) t b_{t}}{(\log t)^{2}}\right\}=-(2 \pi)^{-2} \operatorname{det}(\Gamma)^{-1 / 2} \kappa(2,2)^{-4}(\lambda-\varepsilon) .
$$

Combining (4.36), (4.39) and (4.46) and letting $\varepsilon \rightarrow 0$ we obtain

$$
\begin{aligned}
& \limsup _{t \rightarrow \infty} \frac{1}{b_{t}} \log \mathbb{P}\left\{|\mathbb{E}| Z[0, t]|-| Z[0, t]|| \geq \frac{\lambda t b_{t}}{(\log t)^{2}}\right\} \\
& \leq-(2 \pi)^{-2} \operatorname{det}(\Gamma)^{-1 / 2} \kappa(2,2)^{-4} \lambda
\end{aligned}
$$


By Varadhan's integral lemma [13, Section 4.3]

$$
\begin{gathered}
\lim \sup _{t \rightarrow \infty} \frac{1}{b_{t}} \log \mathbb{E} \exp \left\{\theta \sqrt{\frac{b_{t}}{t}}(\log t)|\mathbb{E}| Z[0, t]|-| Z[0, t]||^{1 / 2}\right\} \\
\leq \sup _{\lambda>0}\left\{\theta \lambda^{1 / 2}-(2 \pi)^{-2} \operatorname{det}(\Gamma)^{-1 / 2} \kappa(2,2)^{-4} \lambda\right\} \\
=(\theta \pi)^{2} \sqrt{\operatorname{det}(\Gamma)} \kappa(2,2)^{4}
\end{gathered}
$$

(The uniform exponential integrability is provided by Lemma 4.2.)

We now prove the lower bound. Using induction on $N$, one can see that

$$
\begin{aligned}
& A_{t}(\varepsilon)=: \Lambda_{\varepsilon}\left(\frac{t}{b_{t}}\right)^{-2} \sum_{x \in \mathbb{Z}^{2}}\left[\sum_{y \in Z[0, t]} h_{\varepsilon}\left(\sqrt{\frac{b_{t}}{t}}(x-y)\right)\right]^{2} \\
& \leq \Lambda_{\varepsilon}\left(\frac{t}{b_{t}}\right)^{-2} \sum_{k=1}^{2^{N}} \sum_{x \in \mathbb{Z}^{2}}\left[\sum_{y \in Z\left[\frac{k-1}{2^{N}} t, \frac{k}{2^{N}} t\right]} h_{\varepsilon}\left(\sqrt{\frac{b_{t}}{t}}(x-y)\right)\right]^{2} \\
& +2 \Lambda_{\varepsilon}\left(\frac{t}{b_{t}}\right)^{-2} \sum_{j=1}^{N} \sum_{k=1}^{2^{j-1}} \sum_{x \in \mathbb{Z}^{2}}\left[\sum_{y \in Z\left[\frac{2 k-2}{2^{j}} t, \frac{2 k-1}{2^{j}} t\right]} h_{\varepsilon}\left(\sqrt{\frac{b_{t}}{t}}(x-y)\right)\right] \\
& \times\left[\sum_{y^{\prime} \in Z\left[\frac{2 k-1}{2^{j}} t, \frac{2 k}{2^{j}} t\right]} h_{\varepsilon}\left(\sqrt{\frac{b_{t}}{t}}\left(x-y^{\prime}\right)\right)\right] \\
& =: I_{t}(\varepsilon)+2 J_{t}(\varepsilon) .
\end{aligned}
$$

Therefore, with $I_{t}, J_{t}$ given by (4.34)

$$
\begin{aligned}
& \mathbb{E}|Z[0, t]|-|Z[0, t]|=\left(\mathbb{E} I_{t}-I_{t}\right)+J_{t}-\mathbb{E} J_{t} \\
& \quad \geq\left(\mathbb{E} I_{t}-I_{t}\right)+J_{t}(\varepsilon)-\left|J_{t}-J_{t}(\varepsilon)\right|-\mathbb{E} J_{t} \\
& \quad \geq\left(\mathbb{E} I_{t}-I_{t}\right)-\frac{1}{2} I_{t}(\varepsilon)-\left|J_{t}-J_{t}(\varepsilon)\right|-\mathbb{E} J_{t}+\frac{1}{2} A_{t}(\varepsilon) .
\end{aligned}
$$

We will see that the dominant contribution to the lower bound comes from $A_{t}(\varepsilon)$. By the last display we see that

$$
\frac{1}{2} A_{t}(\varepsilon) \leq|\mathbb{E}| Z[0, t]|-| Z[0, t]||+\left|\mathbb{E} I_{t}-I_{t}\right|+\frac{1}{2} I_{t}(\varepsilon)+\left|J_{t}-J_{t}(\varepsilon)\right|+\mathbb{E} J_{t}
$$


and consequently

$$
\begin{aligned}
\left|\frac{1}{2} A_{t}(\varepsilon)\right|^{1 / 2} \leq & |\mathbb{E}| Z[0, t]|-| Z[0, t]||^{1 / 2}+\left|\mathbb{E} I_{t}-I_{t}\right|^{1 / 2} \\
& +\left|\frac{1}{2} I_{t}(\varepsilon)\right|^{1 / 2}+\left|J_{t}-J_{t}(\varepsilon)\right|^{1 / 2}+\left|\mathbb{E} J_{t}\right|^{1 / 2}
\end{aligned}
$$

Notice that it follows from (4.1) that

$$
\mathbb{E} J_{t} \leq C_{N} \frac{t}{(\log t)^{2}} .
$$

If $\bar{p}$ is such that $p^{-1}+\bar{p}^{-1}=1$, then by the generalized Hölder inequality with $f=\theta \sqrt{\frac{b_{t}}{t}} \log t$ we have

$$
\begin{aligned}
& \left\|\exp \frac{f}{p}\left|\frac{1}{2} A_{t}(\varepsilon)\right|^{1 / 2}\right\|_{1} \\
& \leq e^{C_{N} \sqrt{b_{t}}}\left\|\exp \frac{f}{p}|\mathbb{E}| Z[0, t]|-| Z[0, t]||^{1 / 2}\right\|_{p} \cdot\left\|\exp \frac{f}{p}\left|\mathbb{E} I_{t}-I_{t}\right|^{1 / 2}\right\|_{3 \bar{p}} \\
& \cdot\left\|\exp \frac{f}{p}\left|\frac{1}{2} I_{t}(\varepsilon)\right|^{1 / 2}\right\|_{3 \bar{p}} \cdot\left\|\exp \frac{f}{p}\left|J_{t}-J_{t}(\varepsilon)\right|^{1 / 2}\right\|_{3 \bar{p}}
\end{aligned}
$$

Taking the $p$-th power and noting that $\bar{p} / p=1 /(p-1)$, this can be rewritten as

$$
\begin{aligned}
\mathbb{E} \exp \{ & \left.\left.\theta \sqrt{\frac{b_{t}}{t}}(\log t)|\mathbb{E}| Z[0, t]|-| Z[0, t]\right|^{1 / 2}\right\} \\
\geq & e^{-C_{N} \sqrt{b_{t}}}\left[\mathbb{E} \exp \left\{\frac{3 \theta}{p-1} \sqrt{\frac{b_{t}}{t}}(\log t)\left|\mathbb{E} I_{t}-I_{t}\right|^{1 / 2}\right\}\right]^{-\frac{p-1}{3}} \\
\times & {\left[\mathbb{E} \exp \left\{\frac{3 \theta}{p-1} \sqrt{\frac{b_{t}}{t}}(\log t) I_{t}(\varepsilon)^{1 / 2}\right\}\right]^{-\frac{p-1}{3}} } \\
\times & {\left[\mathbb{E} \exp \left\{\frac{3 \theta}{p-1} \sqrt{\frac{b_{t}}{t}}(\log t)\left|J_{t}-J_{t}(\varepsilon)\right|^{1 / 2}\right\}\right]^{-\frac{p-1}{3}} } \\
\times & {\left[\mathbb{E} \exp \left\{\frac{\theta}{2 p} \sqrt{\frac{b_{t}}{t}}(\log t)\left|A_{t}(\varepsilon)\right|^{1 / 2}\right\}\right]^{p} }
\end{aligned}
$$


By Lemma 4.6

$$
\begin{aligned}
\lim _{t \rightarrow \infty} \frac{1}{b_{t}} \log \mathbb{E} \exp \left\{\frac{\theta}{2 p} \sqrt{\frac{b_{t}}{t}}(\log t)\left|A_{t}(\varepsilon)\right|^{1 / 2}\right\} \\
=\sup _{g \in \mathcal{F}}\left\{\frac{\pi \theta}{p} \sqrt{\operatorname{det}(\Gamma)}\left(\int_{\mathbb{R}^{2}}\left|\left(g^{2} * h_{\varepsilon}\right)(x)\right|^{2} d x\right)^{1 / 2}\right. \\
\left.-\frac{1}{2} \int_{\mathbb{R}^{2}}\langle\nabla g(x), \Gamma \nabla g(x)\rangle d x\right\} .
\end{aligned}
$$

This will give the main contribution to (4.55). We now bound the other factors in 4.55).

Using Lemma 4.8 together with (4.48) (with $t$ replaced by $2^{-N} t, \theta$ by $2^{-N / 2} \theta$, and $b_{t}$ by $\left.\widetilde{b}_{t}=: b_{2^{N} t}\right)$ we can prove that for any $\theta>0$,

$$
\limsup _{t \rightarrow \infty} \frac{1}{b_{t}} \log \mathbb{E} \exp \left\{\theta \sqrt{\frac{b_{t}}{t}}(\log t)\left|\mathbb{E} I_{t}-I_{t}\right|^{1 / 2}\right\} \leq 2^{-N} C \theta^{2} .
$$

Using (4.20) and Lemma 4.8, we see that

$$
\limsup _{t \rightarrow \infty} \frac{1}{b_{t}} \log \mathbb{E} \exp \left\{\theta \sqrt{\frac{b_{t}}{t}}(\log t) I_{t}(\varepsilon)^{1 / 2}\right\} \leq 2^{-N} C \theta^{2},
$$

where $C>0$ does not depend on $\varepsilon$. Notice that

$$
\left|J_{t}-J_{t}(\varepsilon)\right| \leq \sum_{j=1}^{N} \sum_{k=1}^{2^{j-1}}\left|K_{j, k}(\varepsilon)\right|,
$$

where

$$
\begin{aligned}
K_{j, k}(\varepsilon)=\mid Z\left[\frac{2 k-2}{2^{j}} t\right. & \left., \frac{2 k-1}{2^{j}} t\right] \cap Z\left[\frac{2 k-1}{2^{j}} t, \frac{2 k}{2^{j}} t\right] \mid \\
-\Lambda_{\varepsilon}\left(\frac{t}{b_{t}}\right)^{-2} \sum_{x \in \mathbb{Z}^{2}} & {\left[\sum_{y \in Z\left[\frac{2 k-2}{2^{j}} t, \frac{2 k-1}{2^{j}} t\right]} h_{\varepsilon}\left(\sqrt{\frac{b_{t}}{t}}(x-y)\right)\right] } \\
& \times\left[\sum_{y^{\prime} \in Z\left[\frac{2 k-1}{2^{j}} t, \frac{2 k}{2^{j}} t\right]} h_{\varepsilon}\left(\sqrt{\frac{b_{t}}{t}}\left(x-y^{\prime}\right)\right)\right] .
\end{aligned}
$$


For each $1 \leq j \leq N, K_{j, 1}(\varepsilon), \cdots, K_{j, 2^{N-1}}(\varepsilon)$ forms an i.i.d sequence with the same distribution as $B_{t}^{(j)}(\varepsilon)$. It then follows from Lemma 4.7 and Hölder's inequality that

$$
\limsup _{\varepsilon \rightarrow 0} \limsup _{t \rightarrow \infty} \frac{1}{b_{t}} \log \mathbb{E} \exp \left\{\theta \sqrt{\frac{b_{t}}{t}}(\log t)\left|J_{t}-J_{t}(\varepsilon)\right|^{1 / 2}\right\}=0 .
$$

Hence

$$
\begin{aligned}
\liminf _{t \rightarrow \infty} \frac{1}{b_{t}} & \log \mathbb{E} \exp \left\{\theta \sqrt{\frac{b_{t}}{t}}(\log t)|\mathbb{E}| Z[0, t]|-| Z[0, t]||^{1 / 2}\right\} \\
\geq & -2^{-N+1} C \frac{p-1}{3}\left(\frac{3 \theta}{p-1}\right)^{2} \\
& -\frac{p-1}{3} \limsup _{t \rightarrow \infty} \frac{1}{b_{t}} \log \mathbb{E} \exp \left\{\frac{3 \theta}{p-1} \sqrt{\frac{b_{t}}{t}} \log t\left|J_{t}-J_{t}(\varepsilon)\right|^{1 / 2}\right\} \\
& +p \sup _{g \in \mathcal{F}}\left\{\frac{\pi \theta}{p} \sqrt{\operatorname{det}(\Gamma)}\left(\int_{\mathbb{R}^{2}}\left|\left(g^{2} * h_{\varepsilon}\right)(x)\right|^{2} d x\right)^{1 / 2}\right. \\
& \left.-\frac{1}{2} \int_{\mathbb{R}^{2}}\langle\nabla g(x), \Gamma \nabla g(x)\rangle d x\right\} .
\end{aligned}
$$

Take limits on the right hand side in the following order: let $\varepsilon \rightarrow 0^{+}$, (using (4.61) ), $N \rightarrow \infty$, and then $p \rightarrow 1^{+}$. We obtain

$$
\begin{aligned}
\liminf _{t \rightarrow \infty} & \frac{1}{b_{t}} \log \mathbb{E} \exp \left\{\theta \sqrt{\frac{b_{t}}{t}}(\log t)|\mathbb{E}| Z[0, t]|-| Z[0, t]||^{1 / 2}\right\} \\
\geq & \sup _{g \in \mathcal{F}}\left\{\pi \theta \sqrt{\operatorname{det}(\Gamma)}\left(\int_{\mathbb{R}^{2}}|g(x)|^{4} d x\right)^{1 / 2}-\frac{1}{2} \int_{\mathbb{R}^{2}}\langle\nabla g(x), \Gamma \nabla g(x)\rangle d x\right\} \\
& =(\pi \theta)^{2} \sqrt{\operatorname{det}(\Gamma)} \sup _{f \in \mathcal{F}}\left\{\left(\int_{\mathbb{R}^{2}}|f(x)|^{4} d x\right)^{1 / 2}-\frac{1}{2} \int_{\mathbb{R}^{2}}|\nabla f(x)|^{2} d x\right\} \\
& =(\pi \theta)^{2} \sqrt{\operatorname{det}(\Gamma)} \kappa(2,2)^{4},
\end{aligned}
$$

where the second step follows from the substitution $g(x)=\sqrt{|\operatorname{det}(A)|} f(A x)$ with the $2 \times 2$ matrix $A$ satisfying

$$
A^{\tau} \Gamma A=(\pi \theta)^{2} \sqrt{\operatorname{det}(\Gamma)} I_{2 \times 2}
$$


( $I_{2 \times 2}$ is the $2 \times 2$ identity matrix), and where the last step follows from Lemma A.2 in [7].

\section{Exponential asymptotics for the smoothed range}

In order to prove Lemma 4.6 we first obtain a weak convergence result.

Let $\beta>0$ and write

$$
A_{t, \beta}(\varepsilon)=: \Lambda_{\varepsilon}(t)^{-2} \sum_{x \in \mathbb{Z}^{2}}\left[\sum_{y \in Z[0, \beta t]} h_{\varepsilon}\left(\frac{x-y}{\sqrt{t}}\right)\right]^{2}
$$

and

$$
B_{t, \beta}(\varepsilon)=: \Lambda_{\varepsilon}(t)^{-2} \sum_{x \in \mathbb{Z}^{2}}\left[\sum_{y \in Z[0, \beta t]} h_{\varepsilon}\left(\frac{x-y}{\sqrt{t}}\right)\right]\left[\sum_{y^{\prime} \in Z^{\prime}[0, \beta t]} h_{\varepsilon}\left(\frac{x-y^{\prime}}{\sqrt{t}}\right)\right] .
$$

Let $W(t), W^{\prime}(t)$ be independent planar Brownian motions, each with covariance matrix $\Gamma$ and write

$$
\alpha_{\varepsilon}\left([0, t]^{2}\right)=\int_{0}^{t} \int_{0}^{t}\left(h_{\varepsilon} * h_{\varepsilon}\right)\left(W(s)-W^{\prime}(r)\right) d r d s
$$

and

$$
\alpha\left([0, t]^{2}\right)=\lim _{\varepsilon \rightarrow 0} \alpha_{\varepsilon}\left([0, t]^{2}\right) .
$$

\section{Lemma 5.1}

$$
\begin{aligned}
& \frac{(\log t)^{2}}{t}\left[\left|Z[0, \beta t] \cap Z^{\prime}[0, \beta t]\right|-B_{t, \beta}(\varepsilon)\right] \\
& \quad \stackrel{d}{\longrightarrow}(2 \pi)^{2} \operatorname{det}(\Gamma)\left[\alpha\left([0, \beta]^{2}\right)-\alpha_{\varepsilon}\left([0, \beta]^{2}\right)\right]
\end{aligned}
$$

and

$$
\frac{(\log t)^{2}}{t} A_{t, \beta}(\varepsilon) \stackrel{d}{\longrightarrow}(2 \pi)^{2} \operatorname{det}(\Gamma) \int_{\mathbb{R}^{2}}\left(\int_{0}^{\beta} h_{\varepsilon}(W(s)-x) d s\right)^{2} d x .
$$

as $t \rightarrow \infty$. 
Proof. To prove (5.5), we consider the following result given on p.697 of [23]: if $Z^{(t)}(s)=: \frac{Z(t s)}{\sqrt{t}}$ then

$$
\begin{aligned}
\left(Z^{(t)}(\cdot),\left(Z^{\prime}\right)^{(t)}(\cdot), \frac{(\log t)^{2}}{t}\left|Z[0, \beta t] \cap Z^{\prime}[0, \beta t]\right|\right) \\
\stackrel{d}{\longrightarrow}\left(W(\cdot), W^{\prime}(\cdot),(2 \pi)^{2} \operatorname{det}(\Gamma) \alpha\left([0, \beta]^{2}\right)\right)
\end{aligned}
$$

in the Skorohod topology as $t \rightarrow \infty$. Actually, the proof in 23] is for the discrete time random walk, but a similar proof works for $Z$.

Let $M>0$ be fixed for a moment. Notice that

$$
p_{t, \varepsilon}(x) \equiv \Lambda_{\varepsilon}(t)^{-1} h_{\varepsilon}\left(\frac{x}{\sqrt{t}}\right), \quad x \in \mathbb{Z}^{2},
$$

defines a probability density on $\mathbb{Z}^{2}$ and that

$$
\widehat{p}_{t, \varepsilon}\left(\frac{\lambda}{\sqrt{t}}\right)=\Lambda_{\varepsilon}(t)^{-1} \sum_{x \in \mathbb{Z}^{2}} h_{\varepsilon}\left(\frac{x}{\sqrt{t}}\right) \exp \left\{i \lambda \cdot \frac{x}{\sqrt{t}}\right\} \longrightarrow \widehat{h}_{\varepsilon}(\lambda)
$$

uniformly on $[-M, M]^{2}$ as $t \rightarrow \infty$. Consequently the family

$$
\psi_{t}(x, y)=\int_{[-M, M]^{2}}\left|\widehat{p}_{t, \varepsilon}\left(\frac{\lambda}{\sqrt{t}}\right)\right|^{2}\left[\int_{0}^{\beta} e^{i \lambda \cdot x(s)} d s\right]\left[\int_{0}^{\beta} e^{-i \lambda \cdot y\left(s^{\prime}\right)} d s^{\prime}\right] d \lambda
$$

are convergent continuous functionals on $D\left([0, \beta], \mathbb{R}^{2}\right) \otimes D\left([0, \beta], \mathbb{R}^{2}\right)$. There- 
fore

$$
\begin{aligned}
& \left(\frac{1}{t^{2}} \int_{[-M, M]^{2}}\left|\widehat{p}_{t, \varepsilon}\left(\frac{\lambda}{\sqrt{t}}\right)\right|^{2}\right. \\
& {\left[\int_{0}^{\beta t} \exp \left\{i \lambda \cdot \frac{Z(s)}{\sqrt{t}}\right\} d s\right]\left[\int_{0}^{\beta t} \exp \left\{-i \lambda \cdot \frac{Z^{\prime}\left(s^{\prime}\right)}{\sqrt{t}}\right\} d s^{\prime}\right] d \lambda,} \\
& \left.\frac{(\log t)^{2}}{t}\left|Z[0, \beta t] \cap Z^{\prime}[0, \beta t]\right|\right) \\
& =\left(\int_{[-M, M]^{2}}\left|\widehat{p}_{t, \varepsilon}\left(\frac{\lambda}{\sqrt{t}}\right)\right|^{2}\right. \\
& {\left[\int_{0}^{\beta} \exp \left\{i \lambda \cdot Z^{(t)}(s)\right\} d s\right]\left[\int_{0}^{\beta} \exp \left\{-i \lambda \cdot\left(Z^{\prime}\right)^{(t)}\left(s^{\prime}\right)\right\} d s^{\prime}\right] d \lambda,} \\
& \left.\frac{(\log t)^{2}}{t}\left|Z[0, \beta t] \cap Z^{\prime}[0, \beta t]\right|\right) \\
& \stackrel{d}{\longrightarrow}\left(\int_{[-M, M]^{2}}\left|\widehat{h}_{\varepsilon}(\lambda)\right|^{2}\left[\int_{0}^{\beta} e^{i \lambda \cdot W(s)} d s\right]\left[\int_{0}^{\beta} e^{-i \lambda \cdot W^{\prime}\left(s^{\prime}\right)} d s^{\prime}\right] d \lambda,\right. \\
& \left.(2 \pi)^{2} \operatorname{det}(\Gamma) \alpha\left([0, \beta]^{2}\right)\right)
\end{aligned}
$$

Recall that by Lemma 3 in [8],

$$
\sup _{t} \mathbb{E} \exp \left\{\theta \frac{\log t}{t}|Z[0, t]|\right\}<\infty
$$

for all $\theta>0$. We will show that uniformly in $\lambda \in[-M, M]^{2}$

$$
\begin{aligned}
\lim _{t \rightarrow \infty} \frac{1}{t^{2}} \mathbb{E} \mid \int_{0}^{\beta t} \exp \left\{i \lambda \cdot \frac{Z(s)}{\sqrt{t}}\right\} d s \\
\quad-\left.\frac{\log t}{2 \pi \sqrt{\operatorname{det}(\Gamma)}} \sum_{x \in Z[0, \beta t]} \exp \left\{i \lambda \cdot \frac{x}{\sqrt{t}}\right\}\right|^{2}=0 .
\end{aligned}
$$

Using the inequality

$$
\left|A A^{\prime}-B B^{\prime}\right| \leq\left|A\left(B-B^{\prime}\right)\right|+\left|(A-B) B^{\prime}\right|,
$$

the Cauchy-Schwarz inequality and (5.12), we see from (5.13) that uniformly 
in $\lambda \in[-M, M]^{2}$

$$
\begin{aligned}
& \lim _{t \rightarrow \infty} \frac{1}{t^{2}} \mathbb{E} \mid\left[\int_{0}^{\beta t} \exp \left\{i \lambda \cdot \frac{Z(s)}{\sqrt{t}}\right\} d s\right]\left[\int_{0}^{\beta t} \exp \left\{-i \lambda \cdot \frac{Z^{\prime}\left(s^{\prime}\right)}{\sqrt{t}}\right\} d s^{\prime}\right] \\
& -\left(\frac{\log t}{2 \pi \sqrt{\operatorname{det}(\Gamma)}}\right)^{2}\left[\sum_{x \in Z[0, \beta t]} \exp \left\{i \lambda \cdot \frac{x}{\sqrt{t}}\right\}\right] \\
& \qquad\left[\sum_{x \in Z^{\prime}[0, \beta t]} \exp \left\{i \lambda \cdot \frac{x^{\prime}}{\sqrt{t}}\right\}\right] \mid \\
& =0 .
\end{aligned}
$$

Together with (5.11) this shows that

$$
\begin{aligned}
& \left(\left(\frac{\log t}{2 \pi t}\right)^{2} \int_{[-M, M]^{2}}\left|\widehat{p}_{t, \varepsilon}\left(\frac{\lambda}{\sqrt{t}}\right)\right|^{2}\right. \\
& {\left[\sum_{x \in Z[0, \beta t]} \exp \left\{i \lambda \cdot \frac{x}{\sqrt{t}}\right\}\right]\left[\sum_{x^{\prime} \in Z^{\prime}[0, \beta t]} \exp \left\{-i \lambda \cdot \frac{x^{\prime}}{\sqrt{t}}\right\}\right] d \lambda,} \\
& \left.\frac{(\log t)^{2}}{t}\left|Z[0, \beta t] \cap Z^{\prime}[0, \beta t]\right|\right) \\
& \stackrel{d}{\longrightarrow}\left(\operatorname{det}(\Gamma) \int_{[-M, M]^{2}}\left|\widehat{h}_{\varepsilon}(\lambda)\right|^{2}\left[\int_{0}^{\beta} e^{i \lambda \cdot W(s)} d s\right]\left[\int_{0}^{\beta} e^{-i \lambda \cdot W^{\prime}\left(s^{\prime}\right)} d s^{\prime}\right] d \lambda,\right. \\
& \left.(2 \pi)^{2} \operatorname{det}(\Gamma) \alpha\left([0, \beta]^{2}\right)\right) \text {. }
\end{aligned}
$$

Notice by (5.9) that for any $\delta>0$, one can take $M>0$ sufficiently large so that

$$
\left|\widehat{p}_{t, \varepsilon}\left(\frac{\lambda}{\sqrt{t}}\right)\right|<\delta, \quad \lambda \in[-\sqrt{t} \pi, \sqrt{t} \pi]^{2} \backslash[M, M]^{2},
$$

if $t$ is sufficiently large. Consequently

$$
\begin{aligned}
& H_{t}=:\left.\left|\int_{[-\sqrt{t} \pi, \sqrt{t} \pi]^{2} \backslash[-M, M]^{2}}\right| \widehat{p}_{t, \varepsilon}\left(\frac{\lambda}{\sqrt{t}}\right)\right|^{2}\left[\sum_{x \in Z[0, \beta t]} \exp \left\{i \lambda \cdot \frac{x}{\sqrt{t}}\right\}\right] \\
& \times\left[\sum_{x^{\prime} \in Z^{\prime}[0, \beta t]} \exp \left\{-i \lambda \cdot \frac{x^{\prime}}{\sqrt{t}}\right\}\right] d \lambda \mid \\
& \leq(2 \pi)^{2} \delta t\left|Z[0, \beta t] \cap Z^{\prime}[0, \beta t]\right| .
\end{aligned}
$$


It follows from (4.1) that $(\log t /(2 \pi t))^{2} H_{t} \rightarrow 0$ in $L^{1}$ uniformly in large $t$ as $M \rightarrow \infty$. Therefore, using (5.15) and the fact that $\widehat{h} \in L^{2}$, we obtain

$$
\begin{gathered}
\left(\left(\frac{\log t}{2 \pi t}\right)^{2} \int_{[-\sqrt{t} \pi, \sqrt{t} \pi]^{2}}\left|\widehat{p}_{t, \varepsilon}\left(\frac{\lambda}{\sqrt{t}}\right)\right|^{2}\left[\sum_{x \in Z[0, \beta t]} \exp \left\{i \lambda \cdot \frac{x}{\sqrt{t}}\right\}\right]\right. \\
\left.\times\left[\sum_{x^{\prime} \in Z^{\prime}[0, \beta t]} \exp \left\{-i \lambda \cdot \frac{x^{\prime}}{\sqrt{t}}\right\}\right] d \lambda, \frac{(\log t)^{2}}{t}\left|Z[0, \beta t] \cap Z^{\prime}[0, \beta t]\right|\right) \\
\stackrel{d}{\longrightarrow}\left(\left.\operatorname{det}(\Gamma) \int_{\mathbb{R}^{2}} \widehat{h}_{\varepsilon}(\lambda)\right|^{2}\left[\int_{0}^{\beta} e^{i \lambda \cdot W(s)} d s\right]\left[\int_{0}^{\beta} e^{-i \lambda \cdot W^{\prime}\left(s^{\prime}\right)} d s^{\prime}\right] d \lambda,\right. \\
\left.(2 \pi)^{2} \operatorname{det}(\Gamma) \alpha\left([0, \beta]^{2}\right)\right) .
\end{gathered}
$$

Note that

$$
\begin{aligned}
B_{t, \beta}(\varepsilon) & =\sum_{x \in \mathbb{Z}^{2}}\left[\sum_{y \in Z[0, \beta t]} \Lambda_{\varepsilon}(t)^{-1} h_{\varepsilon}\left(\frac{x-y}{\sqrt{t}}\right)\right]\left[\sum_{y^{\prime} \in Z^{\prime}[0, \beta t]} \Lambda_{\varepsilon}(t)^{-1} h_{\varepsilon}\left(\frac{x-y^{\prime}}{\sqrt{t}}\right)\right] \\
& =\sum_{y \in Z[0, \beta t]} \sum_{y^{\prime} \in Z^{\prime}[0, \beta t]}\left[\sum_{x \in \mathbb{Z}^{2}} p_{t, \varepsilon}(x-y) p_{t, \varepsilon}\left(x-y^{\prime}\right)\right] .
\end{aligned}
$$

It then follows from Parseval's identity that

$$
\begin{aligned}
& (2 \pi)^{2} t B_{t, \beta}(\varepsilon) . \\
& =t \int_{[-\pi, \pi]^{2}}\left|\widehat{p}_{t, \varepsilon}(\lambda)\right|^{2}\left[\sum_{y \in Z[0, \beta t]} e^{i \lambda \cdot y}\right]\left[\sum_{y^{\prime} \in Z^{\prime}[0, \beta t]} e^{-i \lambda \cdot y^{\prime}}\right] d \lambda \\
& =\int_{[-\sqrt{t} \pi, \sqrt{t} \pi]^{2}}\left|\widehat{p}_{t, \varepsilon}\left(\frac{\lambda}{\sqrt{t}}\right)\right|^{2} \\
& \quad\left[\sum_{y \in Z[0, \beta t]} \exp \left\{i \lambda \cdot \frac{y}{\sqrt{t}}\right\}\right]\left[\sum_{y^{\prime} \in Z^{\prime}[0, \beta t]} \exp \left\{-i \lambda \cdot \frac{y^{\prime}}{\sqrt{t}}\right\}\right] d \lambda .
\end{aligned}
$$

Similarly, using the fact that $h_{\varepsilon}$ is symmetric so that $\widehat{h}_{\varepsilon}(\lambda)$ is real

$$
\int_{\mathbb{R}^{2}}\left|\widehat{h}_{\varepsilon}(\lambda)\right|^{2}\left[\int_{0}^{\beta} e^{i \lambda \cdot W(s)} d s\right]\left[\int_{0}^{\beta} e^{-i \lambda \cdot W^{\prime}\left(s^{\prime}\right)} d s^{\prime}\right] d \lambda=\alpha_{\varepsilon}\left([0, \beta]^{2}\right) .
$$


Thus, we have proved

$$
\begin{aligned}
& \left(\frac{(\log t)^{2}}{t} B_{t, \beta}(\varepsilon), \frac{(\log t)^{2}}{t}\left|Z[0, \beta t] \cap Z^{\prime}[0, \beta t]\right|\right) \\
& \stackrel{d}{\longrightarrow}\left((2 \pi)^{2} \operatorname{det}(\Gamma) \alpha_{\varepsilon}\left([0, \beta]^{2}\right), \quad(2 \pi)^{2} \operatorname{det}(\Gamma) \alpha\left([0, \beta]^{2}\right)\right) .
\end{aligned}
$$

(15.5) follows from this.

Thus to complete the proof of (5.5) it only remains to show (5.13) uniformly in $\lambda \in[-M, M]^{2}$. We will show that for any $\delta>0$ we can find $\delta^{\prime}>0$ and $t_{0}<\infty$ such that

$$
\frac{1}{t^{2}} \mathbb{E}\left|\int_{0}^{\beta t} \exp \left\{i \lambda \cdot \frac{Z(s)}{\sqrt{t}}\right\} d s-\int_{0}^{\beta t} \exp \left\{i \gamma \cdot \frac{Z(s)}{\sqrt{t}}\right\} d s\right|^{2}<\delta
$$

and

$$
\left(\frac{\log t}{t}\right)^{2} \mathbb{E}\left|\sum_{x \in Z[0, \beta t]} \exp \left\{i \lambda \cdot \frac{x}{\sqrt{t}}\right\}-\sum_{x \in Z[0, \beta t]} \exp \left\{i \gamma \cdot \frac{x}{\sqrt{t}}\right\}\right|^{2}<\delta
$$

for all $t \geq t_{0}$ and $|\lambda-\gamma| \leq \delta^{\prime}$. We then cover $[-M, M]^{2}$ by a finite number of discs $B\left(\lambda_{k}, \delta^{\prime}\right)$ of radius $\delta^{\prime}$ centered at $\lambda_{k}, k=1, \ldots, N$. Define $\tau(\lambda)=\lambda_{k}$ where $k$ is the smallest integer with $\lambda \in B\left(\lambda_{k}, \delta^{\prime}\right)$. By [8, (4.11)], we can choose $t_{1}<\infty$ such that for all $t \geq t_{1}$ and $k=1, \ldots, N$,

$$
\frac{1}{t^{2}} \mathbb{E}\left|\int_{0}^{\beta t} \exp \left\{i \lambda_{k} \cdot \frac{Z(s)}{\sqrt{t}}\right\} d s-\frac{\log t}{2 \pi \sqrt{\operatorname{det}(\Gamma)}} \sum_{x \in Z[0, \beta t]} \exp \left\{i \lambda_{k} \cdot \frac{x}{\sqrt{t}}\right\}\right|^{2} \leq \delta .
$$

Hence, uniformly in $\lambda \in[-M, M]^{2}$ we have that for all $t \geq t_{0} \vee t_{1}$

$$
\frac{1}{t^{2}} \mathbb{E}\left|\int_{0}^{\beta t} \exp \left\{i \lambda \cdot \frac{Z(s)}{\sqrt{t}}\right\} d s-\frac{\log t}{2 \pi \sqrt{\operatorname{det}(\Gamma)}} \sum_{x \in Z[0, \beta t]} \exp \left\{i \lambda \cdot \frac{x}{\sqrt{t}}\right\}\right|^{2} \leq 3 \delta
$$

proving that (5.13) holds uniformly in $\lambda \in[-M, M]^{2}$. 
(5.23) actually holds uniformly in $t$. To see this note that

$$
\begin{aligned}
& \frac{1}{t^{2}} \mathbb{E}\left|\int_{0}^{\beta t} \exp \left\{i \lambda \cdot \frac{Z(s)}{\sqrt{t}}\right\} d s-\int_{0}^{\beta t} \exp \left\{i \gamma \cdot \frac{Z(s)}{\sqrt{t}}\right\} d s\right|^{2} \\
& \leq \frac{1}{t^{2}} \mathbb{E}\left|\int_{0}^{\beta t}\right| \lambda-\gamma\left|\frac{|Z(s)|}{\sqrt{t}} d s\right|^{2} \\
& =\frac{|\lambda-\gamma|^{2}}{t^{3}} \mathbb{E} \int_{0}^{\beta t} \int_{0}^{\beta t}|Z(s)||Z(r)| d s d r \\
& \leq C \frac{|\lambda-\gamma|^{2}}{t^{3}} \int_{0}^{\beta t} \int_{0}^{\beta t} s^{1 / 2} r^{1 / 2} d s d r \leq C^{\prime}|\lambda-\gamma|^{2} .
\end{aligned}
$$

As for (5.24),

$$
\begin{aligned}
& \mathbb{E}\left|\sum_{x \in Z[0, \beta t]} \exp \left\{i \lambda \cdot \frac{x}{\sqrt{t}}\right\}-\sum_{x \in Z[0, \beta t]} \exp \left\{i \gamma \cdot \frac{x}{\sqrt{t}}\right\}\right|^{2} \\
& \leq 4 \mathbb{E}\left\{|Z[0, \beta t]|^{2} 1_{\left\{\sup _{s \leq \beta t}|Z(s)| \geq C \sqrt{t}\right\}}\right\} \\
& +|\lambda-\gamma|^{2} \mathbb{E}\left\{\left|\sum_{x \in Z[0, \beta t]} \frac{|x|}{\sqrt{t}}\right|^{2} 1_{\left\{\sup _{s \leq \beta t}|Z(s)| \leq C \sqrt{t}\right\}}\right\} \\
& \leq 4 \mathbb{E}\left\{|Z[0, \beta t]|^{2} 1_{\left\{\sup _{s \leq \beta t}|Z(s)| \geq C \sqrt{t}\right\}}\right\}+C^{2}|\lambda-\gamma|^{2} \mathbb{E}|Z[0, \beta t]|^{2}
\end{aligned}
$$

and by (5.12)

$$
\begin{aligned}
& 4 \mathbb{E}\left\{|Z[0, \beta t]|^{2} 1_{\left\{\sup _{s \leq \beta t}|Z(s)| \geq C \sqrt{t}\right\}}\right\}+C^{2}|\lambda-\gamma|^{2} \mathbb{E}|Z[0, \beta t]|^{2} \\
\leq & 4\left\{\mathbb{E}\left(|Z[0, \beta t]|^{4}\right) P\left(\sup _{s \leq \beta t}|Z(s)| \geq C \sqrt{t}\right)\right\}^{1 / 2}+C^{2}|\lambda-\gamma|^{2} \mathbb{E}|Z[0, \beta t]|^{2} \\
\leq & \left(\frac{c t}{\log t}\right)^{2}\left(4\left\{P\left(\sup _{s \leq \beta t}|Z(s)| \geq C \sqrt{t}\right)\right\}^{1 / 2}+C^{2}|\lambda-\gamma|^{2}\right)
\end{aligned}
$$

Taking $C$ large and then choosing $\delta^{\prime}>0$ sufficiently small completes the proof of (5.24) and hence of (5.5). 
We now prove (5.6). Using the facts that $\Lambda_{\varepsilon}(t) \sim t$, that

$$
\begin{array}{r}
\frac{1}{t} \sum_{x \in \mathbb{Z}^{2}}\left[\sum_{y \in Z[0, \beta t]} h_{\varepsilon}\left(\frac{x-y}{\sqrt{t}}\right)\right]^{2}-\int_{\mathbb{R}^{2}}\left[\sum_{y \in Z[0, \beta t]} h_{\varepsilon}\left(x-\frac{y}{\sqrt{t}}\right)\right]^{2} d x \\
=o(1)|Z[0, \beta t]|^{2}
\end{array}
$$

(where the boundedness and continuity of $h_{\varepsilon}$ is used), and (5.12) we need only show that

$$
\begin{aligned}
\left(\frac{\log t}{t}\right)^{2} \int_{\mathbb{R}^{2}}\left[\sum_{y \in Z[0, \beta t]} h_{\varepsilon}\left(x-\frac{y}{\sqrt{t}}\right)\right]^{2} d x \\
\stackrel{d}{\longrightarrow}(2 \pi)^{2} \operatorname{det}(\Gamma) \int_{\mathbb{R}^{2}}\left(\int_{0}^{\beta} h_{\varepsilon}(W(s)-x) d s\right)^{2} d x .
\end{aligned}
$$

By the Parseval identity,

$$
\begin{aligned}
\int_{\mathbb{R}^{2}} & {\left[\sum_{y \in Z[0, \beta t]} h_{\varepsilon}\left(x-\frac{y}{\sqrt{t}}\right)\right]^{2} d x } \\
= & (2 \pi)^{-2} \int_{\mathbb{R}^{2}}\left|\int_{\mathbb{R}^{2}} e^{i \lambda \cdot x} \sum_{y \in Z[0, \beta t]} h_{\varepsilon}\left(x-\frac{y}{\sqrt{t}}\right) d x\right|^{2} d \lambda \\
= & (2 \pi)^{-2} \int_{\mathbb{R}^{2}}\left|\int_{\mathbb{R}^{2}} h_{\varepsilon}(x) e^{i \lambda \cdot x} d x\right|^{2}\left|\sum_{y \in Z[0, \beta t]} \exp \left\{i \lambda \cdot \frac{y}{\sqrt{t}}\right\}\right|^{2} d \lambda \\
= & \int_{\mathbb{R}^{2}}\left|\widehat{h}_{\varepsilon}(\lambda)\right|^{2}\left|\sum_{y \in Z[0, \beta t]} \exp \left\{i \lambda \cdot \frac{y}{\sqrt{t}}\right\}\right|^{2} d \lambda .
\end{aligned}
$$

Let $M>0$ be fixed and $\lambda_{1}, \cdots, \lambda_{N}$ and $\tau$ be defined as above. By 8 , Theorem 7],

$$
\begin{aligned}
& \frac{\log t}{t}\left(\sum_{y \in Z[0, \beta t]} \exp \left\{i \lambda_{1} \cdot \frac{y}{\sqrt{t}}\right\}, \cdots, \sum_{y \in Z[0, \beta t]} \exp \left\{i \lambda_{N} \cdot \frac{y}{\sqrt{t}}\right\}\right) \\
& \stackrel{d}{\longrightarrow}(2 \pi) \sqrt{\operatorname{det}(\Gamma)}\left(\int_{0}^{\beta} e^{i \lambda_{1} \cdot W(s)} d s, \cdots, \int_{0}^{\beta} e^{i \lambda_{N} \cdot W(s)} d s\right) .
\end{aligned}
$$


In particular,

$$
\begin{aligned}
& \left(\frac{\log t}{t}\right)^{2} \int_{[-M, M]^{2}}\left|\widehat{h}_{\varepsilon}(\lambda)\right|^{2}\left|\sum_{y \in Z[0, \beta t]} \exp \left\{i \tau(\lambda) \cdot \frac{y}{\sqrt{t}}\right\}\right|^{2} d \lambda \\
& =\sum_{k=1}^{N} \int_{B_{k}}\left|\widehat{h}_{\varepsilon}(\lambda)\right|^{2}\left|\frac{\log t}{t} \sum_{y \in Z[0, \beta t]} \exp \left\{i \lambda_{k} \cdot \frac{y}{\sqrt{t}}\right\}\right|^{2} d \lambda \\
& \stackrel{d}{\longrightarrow}(2 \pi)^{2} \operatorname{det}(\Gamma) \sum_{k=1}^{N} \int_{B_{k}}\left|\widehat{h}_{\varepsilon}(\lambda)\right|^{2}\left|\int_{0}^{\beta} e^{i \lambda_{k} \cdot W(s)} d s\right|^{2} d \lambda \\
& =(2 \pi)^{2} \operatorname{det}(\Gamma) \int_{[-M, M]^{2}}\left|\widehat{h}_{\varepsilon}(\lambda)\right|^{2}\left|\int_{0}^{\beta} e^{i \tau(\lambda) \cdot W(s)} d s\right|^{2} d \lambda .
\end{aligned}
$$

Notice that the right hand side of (5.34) converges to

$$
(2 \pi)^{2} \operatorname{det}(\Gamma) \int_{[-M, M]^{2}}\left|\widehat{h}_{\varepsilon}(\lambda)\right|^{2}\left|\int_{0}^{\beta} e^{i \lambda \cdot W(s)} d s\right|^{2} d \lambda
$$

as $N \rightarrow \infty$. Applying (5.24) to the left hand side of (5.34) gives

$$
\begin{aligned}
& \left(\frac{\log t}{t}\right)^{2} \int_{[-M, M]^{2}}\left|\widehat{h}_{\varepsilon}(\lambda)\right|^{2}\left|\sum_{y \in Z[0, \beta t]} \exp \left\{i \lambda \cdot \frac{y}{\sqrt{t}}\right\}\right|^{2} d \lambda \\
& \stackrel{d}{\longrightarrow}(2 \pi)^{2} \operatorname{det}(\Gamma) \int_{[-M, M]^{2}}\left|\widehat{h}_{\varepsilon}(\lambda)\right|^{2}\left|\int_{0}^{\beta} e^{i \lambda \cdot W(s)} d s\right|^{2} d \lambda .
\end{aligned}
$$

As $M \rightarrow \infty$, the right hand side of (5.36) converges to

$$
\begin{aligned}
& (2 \pi)^{2} \operatorname{det}(\Gamma) \int_{\mathbb{R}^{2}}\left|\widehat{h}_{\varepsilon}(\lambda)\right|^{2}\left|\int_{0}^{\beta} e^{i \lambda \cdot W(s)} d s\right|^{2} d \lambda \\
& =\operatorname{det}(\Gamma) \int_{\mathbb{R}^{2}}\left(\int_{0}^{\beta} h_{\epsilon}(W(s)-x) d s\right)^{2} d x
\end{aligned}
$$

by Parseval's identity. Note

$$
\begin{aligned}
& H_{t, M}^{\prime}=: \int_{\mathbb{R}^{2} \backslash[-M, M]^{2}}\left|\widehat{h}_{\varepsilon}(\lambda)\right|^{2}\left|\sum_{y \in Z[0, \beta t]} \exp \left\{i \lambda \cdot \frac{y}{\sqrt{t}}\right\}\right|^{2} d \lambda \\
& \leq|Z[0, \beta t]|^{2} \int_{\mathbb{R}^{2} \backslash[-M, M]^{2}}\left|\widehat{h}_{\varepsilon}(\lambda)\right|^{2} d \lambda .
\end{aligned}
$$


It follows from (15.12) and the fact that $\widehat{h}_{\varepsilon} \in L^{2}$ that $\left(\frac{\log t}{2 \pi t}\right)^{2} H_{t, M}^{\prime} \rightarrow 0$ in $L^{1}$ as $M \rightarrow \infty$ uniformly in $t$. Therefore, using the last three displays, we obtain

$$
\begin{aligned}
& \left(\frac{\log t}{t}\right)^{2} \int_{\mathbb{R}^{2}}\left|\widehat{h}_{\varepsilon}(\lambda)\right|^{2}\left|\sum_{y \in Z[0, \beta t]} \exp \left\{i \lambda \cdot \frac{y}{\sqrt{t}}\right\}\right|^{2} d \lambda \\
& \stackrel{d}{\longrightarrow} \operatorname{det}(\Gamma) \int_{\mathbb{R}^{2}}\left(\int_{0}^{\beta} h_{\epsilon}(W(s)-x) d s\right)^{2} d x .
\end{aligned}
$$

Proof of Lemma 4.6. Let $T>0$ be fixed for the moment. Write $\gamma_{t}=t /\left[T^{-1} b_{t}\right]$. We have

$$
\begin{aligned}
& \mathbb{E} \exp \left\{\theta \sqrt{\frac{b_{t}}{t}}(\log t)\left(\Lambda_{\varepsilon}\left(\frac{t}{b_{t}}\right)^{-2} \sum_{x \in \mathbb{Z}^{2}}\left[\sum_{y \in Z[0, t]} h_{\varepsilon}\left(\sqrt{\frac{b_{t}}{t}}(x-y)\right)\right]^{2}\right)^{1 / 2}\right\} \\
& \leq\left[\mathbb { E } \operatorname { e x p } \left\{\theta \sqrt{\frac{b_{t}}{t}}(\log t)\right.\right. \\
& \left.\left.\times\left(\Lambda_{\varepsilon}\left(\frac{t}{b_{t}}\right)^{-2} \sum_{x \in \mathbb{Z}^{2}}\left[\sum_{y \in Z\left[0, \gamma_{t}\right]} h_{\varepsilon}\left(\sqrt{\frac{b_{t}}{t}}(x-y)\right)\right]^{2}\right)^{1 / 2}\right\}\right]^{\left[T^{-1} b_{t}\right]}
\end{aligned}
$$

We obtain from Lemma 5.1 (with $t$ being replaced by $t / b_{t}$ and $\beta=T$ )

$$
\begin{aligned}
& \frac{b_{t}}{t}(\log t)^{2} \Lambda_{\varepsilon}\left(\frac{t}{b_{t}}\right)^{-2} \sum_{x \in \mathbb{Z}^{2}}\left[\sum_{y \in Z\left[0, \gamma_{t}\right]} h_{\varepsilon}\left(\sqrt{\frac{b_{t}}{t}}(x-y)\right)\right]^{2} \\
& \stackrel{d}{\longrightarrow}(2 \pi)^{2} \operatorname{det}(\Gamma) \int_{\mathbb{R}^{2}}\left(\int_{0}^{T} h_{\varepsilon}(W(s)-x) d s\right)^{2} d x, \quad t \rightarrow \infty .
\end{aligned}
$$


In addition,

$$
\begin{aligned}
& \frac{b_{t}}{t}(\log t)^{2} \Lambda_{\varepsilon}\left(\frac{t}{b_{t}}\right)^{-2} \sum_{x \in \mathbb{Z}^{2}}\left[\sum_{y \in Z\left[0, \gamma_{t}\right]} h_{\varepsilon}\left(\sqrt{\frac{b_{t}}{t}}(x-y)\right)\right]^{2} \\
& \quad \leq \frac{b_{t}}{t}(\log t)^{2} \Lambda_{\varepsilon}\left(\frac{t}{b_{t}}\right)^{-2}\|h\|_{\infty}\left|Z\left[0, \gamma_{t}\right]\right| \sum_{\substack{x \in \mathbb{Z}^{2} \\
y \in Z\left[0, \gamma_{t}\right]}} h_{\varepsilon}\left(\sqrt{\frac{b_{t}}{t}}(x-y)\right) \\
& \quad=\frac{b_{t}}{t}(\log t)^{2} \Lambda_{\varepsilon}\left(\frac{t}{b_{t}}\right)^{-2}|| h \|_{\infty}\left|Z\left[0, \gamma_{t}\right]\right|^{2} \sum_{x \in \mathbb{Z}^{2}} h_{\varepsilon}\left(\sqrt{\frac{b_{t}}{t}} x\right) \\
& \quad \leq C\left(\frac{b_{t}}{t}\right)^{2}(\log t)^{2}\left|Z\left[0, \gamma_{t}\right]\right|^{2},
\end{aligned}
$$

where in the last step we used (4.17). (5.12) together with (5.41) then implies that

$$
\begin{aligned}
& \mathbb{E} \exp \left\{\theta \sqrt{\frac{b_{t}}{t}} \Lambda_{\varepsilon}\left(\frac{t}{b_{t}}\right)^{-1}(\log t)\left(\sum_{x \in \mathbb{Z}^{2}}\left[\sum_{y \in Z\left[0, \gamma_{t}\right]} h_{\varepsilon}\left(\sqrt{\frac{b_{t}}{t}}(x-y)\right)\right]^{2}\right)^{1 / 2}\right\} \\
& (5.43) \longrightarrow \mathbb{E} \exp \left\{2 \pi \theta \sqrt{\operatorname{det}(\Gamma)}\left(\int_{\mathbb{R}^{2}}\left(\int_{0}^{T} h_{\varepsilon}(W(s)-x) d s\right)^{2} d x\right)^{1 / 2}\right\} .
\end{aligned}
$$

Combining (5.40) and (15.43) we see that

$$
\begin{aligned}
\limsup _{t \rightarrow \infty} & \frac{1}{b_{t}} \log \mathbb{E} \exp \left\{\theta \sqrt{\frac{b_{t}}{t}}(\log t)\left|A_{t}(\varepsilon)\right|^{1 / 2}\right\} \\
\leq & \frac{1}{T} \log \mathbb{E} \exp \left\{2 \pi \theta \sqrt{\operatorname{det}(\Gamma)}\left(\int_{\mathbb{R}^{2}}\left(\int_{0}^{T} h_{\varepsilon}(W(s)-x) d s\right)^{2} d x\right)^{1 / 2}\right\} .
\end{aligned}
$$

Then the upper bound for (4.19) follows from the fact that

$$
\begin{gathered}
\lim _{T \rightarrow \infty} \frac{1}{T} \log \mathbb{E} \exp \left\{2 \pi \theta \sqrt{\operatorname{det}(\Gamma)}\left(\int_{\mathbb{R}^{2}}\left(\int_{0}^{T} h_{\varepsilon}(W(s)-x) d s\right)^{2} d x\right)^{1 / 2}\right\} \\
=\sup _{g \in \mathcal{F}}\left\{2 \pi \theta \sqrt{\operatorname{det}(\Gamma)}\left(\int_{\mathbb{R}^{2}}\left|\left(g^{2} * h_{\varepsilon}\right)(x)\right|^{2} d x\right)^{1 / 2}\right. \\
\left.-\frac{1}{2} \int_{\mathbb{R}^{2}}\langle\nabla g(x), \Gamma \nabla g(x)\rangle d x\right\} .
\end{gathered}
$$


This is [12, Theorem 7]. (Or see the earlier [7, Theorem 3.1], which uses a slightly different smoothing).

We now prove the lower bound for (4.19). Let $f$ be a smooth function on $\mathbb{R}^{2}$ with compact support and

$$
\|f\|_{2}=\left(\int_{\mathbb{R}^{2}}|f(x)|^{2} d x\right)^{1 / 2}=1 .
$$

We can write

$$
\begin{aligned}
& \sqrt{\frac{b_{t}}{t}}\left(\sum_{x \in \mathbb{Z}^{2}}\left[\sum_{y \in Z[0, t]} h_{\varepsilon}\left(\sqrt{\frac{b_{t}}{t}}(x-y)\right)\right]^{2}\right)^{1 / 2} \\
& =\sqrt{\frac{b_{t}}{t}}\left(\int_{\mathbb{R}^{2}}\left[\sum_{y \in Z[0, t]} h_{\varepsilon}\left(\sqrt{\frac{b_{t}}{t}}([x]-y)\right)\right]^{2} d x\right)^{1 / 2} \\
& =\left(\int_{\mathbb{R}^{2}}\left[\sum_{y \in Z[0, t]} h_{\varepsilon}\left(\sqrt{\frac{b_{t}}{t}}\left(\left[\sqrt{\frac{t}{b_{t}}} x\right]-y\right)\right)\right]^{2} d x\right)^{1 / 2} .
\end{aligned}
$$

Hence by the Cauchy-Schwarz inequality,

$$
\begin{aligned}
\sqrt{\frac{b_{t}}{t}} & \left(\sum_{x \in \mathbb{Z}^{2}}\left[\sum_{y \in Z[0, t]} h_{\varepsilon}\left(\sqrt{\frac{b_{t}}{t}}(x-y)\right)\right]^{2}\right)^{1 / 2} \\
& =\left(\int_{\mathbb{R}^{2}}\left[\sum_{y \in Z[0, t]} h_{\varepsilon}\left(\sqrt{\frac{b_{t}}{t}}\left[\sqrt{\frac{t}{b_{t}}} x\right]-\sqrt{\frac{b_{t}}{t}} y\right)\right]^{2} d x\right)^{1 / 2} \\
& \geq \int_{\mathbb{R}^{2}} f(x) \sum_{y \in Z[0, t]} h_{\varepsilon}\left(\sqrt{\frac{b_{t}}{t}}\left[\sqrt{\frac{t}{b_{t}}} x\right]-\sqrt{\frac{b_{t}}{t}} y\right) d x \\
& =\int_{\mathbb{R}^{2}} f(x) \sum_{y \in Z[0, t]} h_{\varepsilon}\left(x-\sqrt{\frac{b_{t}}{t}} y\right) d x+O(1)|Z[0, t]|, \quad t \rightarrow \infty,
\end{aligned}
$$

where $O(1)$ is bounded by a constant. In view of (4.12), recalling that

$$
\sqrt{\frac{b_{t}}{t}}\left|A_{t}(\varepsilon)\right|^{1 / 2} \sim \frac{b_{t}}{t} \sqrt{\frac{b_{t}}{t}}\left(\sum_{x \in \mathbb{Z}^{2}}\left[\sum_{y \in Z[0, t]} h_{\varepsilon}\left(\sqrt{\frac{b_{t}}{t}}(x-y)\right)\right]^{2}\right)^{1 / 2},
$$

and using Hölder's inequality one can see that the term $O(1)|Z[0, t]|$ does not contribute anything to (4.19). 
By [8, Theorem 8],

$$
\begin{aligned}
\liminf _{t \rightarrow \infty} & \frac{1}{b_{t}} \log \mathbb{E} \exp \left\{\theta \frac{b_{t} \log t}{t} \sum_{y \in Z[0, t]}\left(f * h_{\varepsilon}\right)\left(\sqrt{\frac{b_{t}}{t}} y\right)\right\} \\
\geq & \sup _{g \in \mathcal{F}}\left\{2 \pi \theta \sqrt{\operatorname{det}(\Gamma)} \int_{\mathbb{R}^{2}}\left(f * h_{\varepsilon}\right)(x) g^{2}(x) d x-\frac{1}{2} \int_{\mathbb{R}^{2}}\langle\nabla g(x), \Gamma \nabla g(x)\rangle d x\right\} \\
& =\sup _{g \in \mathcal{F}}\left\{2 \pi \theta \sqrt{\operatorname{det}(\Gamma)} \int_{\mathbb{R}^{2}} f(x)\left(g^{2} * h_{\varepsilon}\right)(x) d x-\frac{1}{2} \int_{\mathbb{R}^{2}}\langle\nabla g(x), \Gamma \nabla g(x)\rangle d x\right\} .
\end{aligned}
$$

We see from (5.47) and (5.48) that

$$
\begin{aligned}
\liminf _{t \rightarrow \infty} & \frac{1}{b_{t}} \log \mathbb{E} \exp \left\{\theta \sqrt{\frac{b_{t}}{t}}(\log t)\left|A_{t}(\varepsilon)\right|^{1 / 2}\right\} \\
\geq \sup _{g \in \mathcal{F}}\left\{2 \pi \theta \sqrt{\operatorname{det}(\Gamma)} \int_{\mathbb{R}^{2}} f(x)\left(g^{2} * h_{\varepsilon}\right)(x) d x\right. & \left.-\frac{1}{2} \int_{\mathbb{R}^{2}}\langle\nabla g(x), \Gamma \nabla g(x)\rangle d x\right\}
\end{aligned}
$$

Taking the supremum over $f$ on the right gives

$$
\begin{aligned}
\liminf _{t \rightarrow \infty} \frac{1}{b_{t}} \log \mathbb{E} \exp \left\{\theta \sqrt{\frac{b_{t}}{t}}(\log t)\left|A_{t}(\varepsilon)\right|^{1 / 2}\right\} \\
\geq \sup _{g \in \mathcal{F}}\left\{2 \pi \theta \sqrt{\operatorname{det}(\Gamma)}\left(\int_{\mathbb{R}^{2}}\left|\left(g^{2} * h_{\varepsilon}\right)(x)\right|^{2} d x\right)^{1 / 2}\right. \\
\left.-\frac{1}{2} \int_{\mathbb{R}^{2}}\langle\nabla g(x), \Gamma \nabla g(x)\rangle d x\right\} .
\end{aligned}
$$

This completes the proof of (4.19).

To prove (4.20), in (4.19) we replace $t$ by $2^{-N} t, \theta$ by $2^{-N / 2} \theta, b_{t}$ by $\widetilde{b}_{t}=: b_{2^{N} t}$ 
and $\varepsilon$ by $2^{N / 2} \varepsilon$ to find that

$$
\begin{aligned}
& \lim _{t \rightarrow \infty} \frac{1}{b_{t}} \log \mathbb{E} \exp \left\{\theta \sqrt{\frac{b_{t}}{t}}(\log t)\right. \\
&\left.\times\left(\Lambda_{\varepsilon}\left(\frac{t}{b_{t}}\right)^{-2} \sum_{x \in \mathbb{Z}^{2}}\left[\sum_{y \in Z\left[0,2^{-N} t\right]} h_{\varepsilon}\left(\sqrt{\frac{b_{t}}{t}}(x-y)\right)\right]^{2}\right)^{1 / 2}\right\} \\
&= \lim _{t \rightarrow \infty} \frac{1}{\widetilde{b}_{2^{-N}}} \log \mathbb{E} \exp \left\{2^{-N / 2} \theta \sqrt{\frac{\widetilde{b}_{2^{-N} t}}{2^{-N} t}}(\log t)\right. \\
&\left.\times\left(\Lambda_{2^{N / 2} \varepsilon}\left(\frac{2^{-N} t}{\widetilde{b}_{2}-N t}\right)^{-2} \sum_{x \in \mathbb{Z}^{2}}\left[\sum_{y \in Z\left[0,2^{-N} t\right]} h_{2^{N / 2} \varepsilon}\left(\sqrt{\frac{\widetilde{b}_{2^{-N} t}}{2^{-N} t}}(x-y)\right)\right]^{2}\right)^{1 / 2}\right\} \\
&= \sup _{g \in \mathcal{F}}\left\{2 \pi 2^{-N / 2} \theta \sqrt{\operatorname{det}(\Gamma)}\left(\int_{\mathbb{R}^{2}}\left|\left(g^{2} * h_{2^{N / 2} \varepsilon}\right)(x)\right|^{2} d x\right)^{1 / 2}\right. \\
&\left.-\frac{1}{2} \int_{\mathbb{R}^{2}}\langle\nabla g(x), \Gamma \nabla g(x)\rangle^{2} d x\right\} \\
& \leq \sup _{g \in \mathcal{F}}\left\{2 \pi 2^{-N / 2} \theta \sqrt{\operatorname{det}(\Gamma)}\left(\int_{\mathbb{R}^{2}}|g(x)|^{4} d x\right)^{1 / 2}\right. \\
&\left.-\frac{1}{2} \int_{\mathbb{R}^{2}}\langle\nabla g(x), \Gamma \nabla g(x)\rangle^{2} d x\right\} . \\
&=\left(2 \pi 2^{-N / 2} \theta\right)^{2} \sqrt{\operatorname{det}(\Gamma)} \sup _{f \in \mathcal{F}}\left\{\left(\int_{\mathbb{R}^{2}}|f(x)|^{4} d x\right)^{1 / 2}\right. \\
&\left.-\frac{1}{2} \int_{\mathbb{R}^{2}}|\nabla f(x)|^{2} d x\right\} . \\
& \pi^{2} \theta^{2} \sqrt{\operatorname{det}(\Gamma)} \kappa(2,2)^{4},
\end{aligned}
$$

where the third step follows from Jensen's inequality, the fourth step follows from the substitution $g(x)=\sqrt{|\operatorname{det}(A)|} f(A x)$ with the $2 \times 2$ matrix $A$ satisfying

$$
A^{\tau} \Gamma A=\left(2 \pi 2^{-N / 2} \theta\right)^{2} \sqrt{\operatorname{det}(\Gamma)} I_{2 \times 2},
$$

and the last step follows from Lemma 7.2 in [7]. 


\section{Exponential approximation}

Let $t_{1}, \cdots, t_{a} \geq 0$ and write

$$
\Delta_{1}=\left[0, t_{1}\right], \text { and } \Delta_{k}=\left[\sum_{j=1}^{k-1} t_{j}, \sum_{j=1}^{k} t_{j}\right] \quad k=2, \cdots, a .
$$

Let $p(x)$ be a positive symmetric function on $\mathbb{Z}^{2}$ with $\sum_{x \in \mathbb{Z}^{2}} p(x)=1$ and define

$$
L=\sum_{j, k=1}^{a}\left[\left|Z\left(\Delta_{j}\right) \cap Z^{\prime}\left(\Delta_{k}\right)\right|-\sum_{x \in \mathbb{Z}^{2}} p(x)\left|Z\left(\Delta_{j}\right) \cap\left(Z^{\prime}\left(\Delta_{k}\right)+x\right)\right|\right],
$$

and

$$
\begin{aligned}
L_{j}= & \left|Z\left[0, t_{j}\right] \cap Z^{\prime}\left[0, t_{j}\right]\right| \\
& -\sum_{x \in \mathbb{Z}^{2}} p(x)\left|Z\left[0, t_{j}\right] \cap\left(Z^{\prime}\left[0, t_{j}\right]+x\right)\right|, \quad j=1, \cdots, a .
\end{aligned}
$$

Lemma 6.1 For any $m \geq 1$,

$$
\mathbb{E} L^{m} \geq 0
$$

and

$$
\left\{\mathbb{E} L^{m}\right\}^{1 / 2} \leq \sum_{\substack{k_{1}+\cdots+k_{a}=m \\ k_{1}, \cdots, k_{a} \geq 0}} \frac{m !}{k_{1} ! \cdots k_{a} !}\left\{\mathbb{E}\left|L_{1}\right|^{k_{1}}\right\}^{1 / 2} \cdots\left\{\mathbb{E}\left|L_{a}\right|^{k_{a}}\right\}^{1 / 2}
$$

Consequently, for any $\theta>0$

$$
\sum_{m=0}^{\infty} \frac{\theta^{m}}{m !}\left\{\mathbb{E} L^{m}\right\}^{1 / 2} \leq \prod_{j=1}^{a} \sum_{m=0}^{\infty} \frac{\theta^{m}}{m !}\left\{\mathbb{E}\left|L_{j}\right|^{m}\right\}^{1 / 2}
$$

Proof. Write

$$
\widehat{p}(\lambda)=\sum_{x \in \mathbb{Z}^{2}} p(x) e^{i \lambda \cdot x}
$$


We note that

$$
|\widehat{p}(\lambda)| \leq \widehat{p}(0)=1
$$

Notice also that

$$
L=\frac{1}{(2 \pi)^{2}} \int_{[-\pi, \pi]^{2}}[1-\widehat{p}(\lambda)]\left[\sum_{j=1}^{a} \sum_{x \in Z\left(\Delta_{j}\right)} e^{i \lambda \cdot x}\right]\left[\sum_{j^{\prime}=1}^{a} \sum_{x^{\prime} \in Z^{\prime}\left(\Delta_{j^{\prime}}\right)} e^{-i \lambda \cdot x^{\prime}}\right] d \lambda .
$$

We therefore have

$$
\begin{aligned}
\mathbb{E} L^{m}= & \frac{1}{(2 \pi)^{2 m}} \int_{\left([-\pi, \pi]^{2}\right)^{m}}\left|\mathbb{E} \prod_{k=1}^{m} \sum_{j=1}^{a} \sum_{x_{k} \in Z\left(\Delta_{j}\right)} e^{i \lambda_{k} \cdot x_{k}}\right|^{2}\left(\prod_{k=1}^{m}\left[1-\widehat{p}\left(\lambda_{k}\right)\right] d \lambda_{k}\right) \\
= & \frac{1}{(2 \pi)^{2 m}} \int_{\left([-\pi, \pi]^{2}\right)^{m}}\left|\sum_{l_{1}, \cdots, l_{m}=1}^{a} \mathbb{E}\left(H_{l_{1}}\left(\lambda_{1}\right) \cdots H_{l_{m}}\left(\lambda_{m}\right)\right)\right|^{2} \\
& \left(\prod_{k=1}^{m}\left[1-\widehat{p}\left(\lambda_{k}\right)\right] d \lambda_{k}\right),
\end{aligned}
$$

where

$$
H_{j}(\lambda)=\sum_{x \in Z\left(\Delta_{j}\right)} e^{i \lambda \cdot x}
$$

This proves (6.4) and implies that

$$
\begin{aligned}
& \left\{\mathbb{E} L^{m}\right\}^{1 / 2} \\
& \leq \frac{1}{(2 \pi)^{m}} \sum_{l_{1}, \cdots, l_{m}=1}^{a}\left\{\int_{\left([-\pi, \pi]^{2}\right)^{m}}\left|\mathbb{E}\left(H_{l_{1}}\left(\lambda_{1}\right) \cdots H_{l_{m}}\left(\lambda_{m}\right)\right)\right|^{2}\right. \\
& \left.\left(\prod_{k=1}^{m}\left[1-\widehat{p}\left(\lambda_{k}\right)\right] d \lambda_{k}\right)\right\}^{1 / 2}
\end{aligned}
$$

Note that for any $k>j$ we can write

$$
H_{k}(\lambda)=\sum_{x \in Z\left(\Delta_{k}\right)} e^{i \lambda \cdot x}=e^{i \lambda \cdot Z\left(t_{j}\right)} H_{k}^{(j)}(\lambda)
$$


where

$$
H_{k}^{(j)}(\lambda)=\sum_{x \in Z\left(\Delta_{k}\right)-Z\left(t_{j}\right)} e^{i \lambda \cdot x}
$$

is independent of $\mathcal{F}_{t_{j}}$.

Let $l_{1}, \cdots, l_{m}$ be fixed and let $k_{j}=\sum_{i=1}^{m} \delta\left(l_{i}, j\right)$ be the number of $l$ 's which are equal to $j$, for each $1 \leq j \leq a$. Then using independence

$$
\begin{aligned}
& \int_{\left([-\pi, \pi]^{2}\right)^{m}}\left|\mathbb{E}\left(H_{l_{1}}\left(\lambda_{1}\right) \cdots H_{l_{m}}\left(\lambda_{m}\right)\right)\right|^{2}\left(\prod_{k=1}^{m}\left[1-\widehat{p}\left(\lambda_{k}\right)\right] d \lambda_{k}\right) \\
& =\int_{\left([-\pi, \pi]^{2}\right)^{m}}\left|\mathbb{E} \prod_{j=1}^{a}\left(H_{j}\left(\lambda_{j, 1}\right) \cdots H_{j}\left(\lambda_{j, k_{j}}\right)\right)\right|^{2}\left(\prod_{j=1}^{a} \prod_{l=1}^{k_{j}}\left[1-\widehat{p}\left(\lambda_{j, l}\right)\right] d \lambda_{j, l}\right) \\
& =\int_{\left([-\pi, \pi]^{2}\right)^{m}} \mid \mathbb{E}\left[\exp \left\{i\left(\sum_{j=2}^{a} \sum_{l=1}^{k_{j}} \lambda_{j, l}\right) \cdot Z\left(t_{1}\right)\right\} \times\left(H_{1}\left(\lambda_{1,1}\right) \cdots H_{1}\left(\lambda_{1, k_{1}}\right)\right)\right] \\
& \mathbb{E}\left(\left.\prod_{j=2}^{a}\left(H_{j}^{(1)}\left(\lambda_{j, 1}\right) \cdots H_{j}^{(1)}\left(\lambda_{j, k_{j}}\right)\right)\right|^{2}\left(\prod_{j=1}^{a} \prod_{l=1}^{k_{j}}\left[1-\widehat{p}\left(\lambda_{j, l}\right)\right] d \lambda_{j, l}\right)\right. \\
& =\int_{\left([-\pi, \pi]^{2}\right)^{m-k_{1}}} \mid \mathbb{E}\left(\left.\prod_{j=2}^{a}\left(H_{j}^{(1)}\left(\lambda_{j, 1}\right) \cdots H_{j}^{(1)}\left(\lambda_{j, k_{j}}\right)\right)\right|^{2}\right. \\
& \\
& F\left(\lambda_{2,1}, \cdots, \lambda_{2, k_{2}} ; \cdots ; \lambda_{a, 1}, \cdots, \lambda_{a, k_{a}}\right)\left(\prod_{j=2}^{a} \prod_{l=1}^{k_{j}}\left[1-\widehat{p}\left(\lambda_{j, l}\right)\right] d \lambda_{j, l}\right),
\end{aligned}
$$

where

$$
\begin{aligned}
& F\left(\lambda_{2,1}, \cdots, \lambda_{2, k_{2}} ; \cdots ; \lambda_{a, 1}, \cdots, \lambda_{a, k_{a}}\right) \\
& =\int_{\left([-\pi, \pi]^{2}\right)^{k_{1}}}\left|\mathbb{E}\left[\exp \left\{i\left(\sum_{j=2}^{a} \sum_{l=1}^{k_{j}} \lambda_{j, l}\right) \cdot Z\left(t_{1}\right)\right\} \times\left(H_{1}\left(\lambda_{1,1}\right) \cdots H_{1}\left(\lambda_{1, k_{1}}\right)\right)\right]\right|^{2} \\
& \quad\left(\prod_{l=1}^{k_{1}}\left[1-\widehat{p}\left(\lambda_{1, l}\right)\right] d \lambda_{1, l}\right) .
\end{aligned}
$$


Notice that by symmetry

$$
\mathbb{E}\left[\exp \left\{i\left(\sum_{j=2}^{a} \sum_{l=1}^{k_{j}} \lambda_{j, l}\right) \cdot Z\left(t_{1}\right)\right\}\left(H_{1}\left(\lambda_{1,1}\right) \cdots H_{1}\left(\lambda_{1, k_{1}}\right)\right)\right]
$$

is real valued. Hence if $Z^{\prime}$ denotes an independent copy of $Z$, and $H_{1}^{\prime}$ is obtained from $H_{1}$ by replacing $Z$ by $Z^{\prime}$,

$$
\begin{gathered}
F\left(\lambda_{2,1}, \cdots, \lambda_{2, k_{2}} ; \cdots ; \lambda_{a, 1}, \cdots, \lambda_{a, k_{a}}\right) \\
=\int_{\left([-\pi, \pi]^{2}\right)^{k_{1}}} \mathbb{E}\left[\exp \left\{i\left(\sum_{j=2}^{a} \sum_{l=1}^{k_{j}} \lambda_{j, l}\right) \cdot\left(Z\left(t_{1}\right)+Z^{\prime}\left(t_{1}\right)\right)\right\}\right. \\
\left.\times \prod_{l=1}^{k_{1}}\left(H_{1}\left(\lambda_{1, l}\right) H_{1}^{\prime}\left(\lambda_{1, l}\right)\right)\right]\left(\prod_{l=1}^{k_{1}}\left[1-\widehat{p}\left(\lambda_{1, l}\right)\right] d \lambda_{1, l}\right) \\
=\mathbb{E}\left[\exp \left\{i\left(\sum_{j=2}^{a} \sum_{l=1}^{k_{j}} \lambda_{j, l}\right) \cdot\left(Z\left(t_{1}\right)+Z^{\prime}\left(t_{1}\right)\right)\right\}\right. \\
\left.\quad \times \int_{\left([-\pi, \pi]^{2}\right)^{k_{1}}} \prod_{l=1}^{k_{1}}\left(H_{1}\left(\lambda_{1, l}\right) H_{1}^{\prime}\left(\lambda_{1, l}\right)\right)\right]\left(\prod_{l=1}^{k_{1}}\left[1-\widehat{p}\left(\lambda_{1, l}\right)\right] d \lambda_{1, l}\right) .
\end{gathered}
$$

By the fact that

$$
\begin{aligned}
& \int_{\left([-\pi, \pi]^{2}\right)^{k_{1}}} \prod_{l=1}^{k_{1}}\left(H_{1}\left(\lambda_{1, l}\right) H_{1}^{\prime}\left(\lambda_{1, l}\right)\right)\left(\prod_{l=1}^{k_{1}}\left[1-\widehat{p}\left(\lambda_{1, l}\right)\right] d \lambda_{1, l}\right) \\
& =\left[\int_{[-\pi, \pi]^{2}}[1-\widehat{p}(\lambda)] H_{1}(\lambda) H_{1}^{\prime}(\lambda) d \lambda\right]^{k_{1}}=(2 \pi)^{2 k_{1}} L_{1}^{k_{1}},
\end{aligned}
$$

we have proved that

$$
\begin{aligned}
& \int_{\left([-\pi, \pi]^{2}\right)^{m}}\left|\mathbb{E}\left(H_{l_{1}}\left(\lambda_{1}\right) \cdots H_{l_{m}}\left(\lambda_{m}\right)\right)\right|^{2}\left(\prod_{k=1}^{m}\left[1-\widehat{p}\left(\lambda_{k}\right)\right] d \lambda_{k}\right) \\
& \leq(2 \pi)^{2 k_{1}} \mathbb{E}\left|L_{1}\right|^{k_{1}} \int_{\left([-\pi, \pi]^{2}\right)^{m-k_{1}}} \mid \mathbb{E}\left(\left.\prod_{j=2}^{a}\left(H_{j}^{(1)}\left(\lambda_{j, 1}\right) \cdots H_{j}^{(1)}\left(\lambda_{j, k_{j}}\right)\right)\right|^{2}\right. \\
& \left(\prod_{j=2}^{a} \prod_{l=1}^{k_{j}}\left[1-\widehat{p}\left(\lambda_{j, l}\right)\right] d \lambda_{j, l}\right) .
\end{aligned}
$$


Repeating the above procedure,

$$
\begin{array}{r}
\int_{\left([-\pi, \pi]^{2}\right)^{m}}\left|\mathbb{E}\left(H_{l_{1}}\left(\lambda_{1}\right) \cdots H_{l_{m}}\left(\lambda_{m}\right)\right)\right|^{2}\left(\prod_{k=1}^{m}\left[1-\widehat{p}\left(\lambda_{k}\right)\right] d \lambda_{k}\right) \\
\leq \prod_{j=1}^{a}\left\{(2 \pi)^{2 k_{j}} \mathbb{E}\left|L_{j}\right|^{k_{j}}\right\}=(2 \pi)^{2 m} \prod_{j=1}^{a} \mathbb{E}\left|L_{j}\right|^{k_{j}}
\end{array}
$$

Our Lemma now follows from (6.12).

Proof of Lemma 4.7 Define

$$
q_{t, \varepsilon}(x)=\Lambda_{\varepsilon}\left(\frac{t}{b_{t}}\right)^{-2} \sum_{z \in \mathbb{Z}^{2}} h_{\varepsilon}\left(\sqrt{\frac{b_{t}}{t}}(x-z)\right) h_{\varepsilon}\left(\sqrt{\frac{b_{t}}{t}} z\right), \quad x \in \mathbb{Z}^{2} .
$$

Then $q_{t, \varepsilon}(x)$ is a probability density on $\mathbb{Z}^{2}$. We claim that

$$
B_{t}^{(0)}(\varepsilon)=\sum_{x \in \mathbb{Z}^{2}} q_{t, \varepsilon}(x)\left|Z[0, t] \cap\left(x+Z^{\prime}[0, t]\right)\right| .
$$

This follows from the fact that

$$
\begin{aligned}
& \sum_{x \in \mathbb{Z}^{2}} \sum_{y \in Z[0, t]} h_{\varepsilon}\left(\sqrt{\frac{b_{t}}{t}}(x-y)\right) \sum_{y^{\prime} \in Z^{\prime}[0, t]} h_{\varepsilon}\left(\sqrt{\frac{b_{t}}{t}}\left(x-y^{\prime}\right)\right) \\
& =\sum_{x \in \mathbb{Z}^{2}} \sum_{y^{\prime} \in Z^{\prime}[0, t]} h_{\varepsilon}\left(\sqrt{\frac{b_{t}}{t}} x\right) \sum_{y \in \mathbb{Z}^{2}} h_{\varepsilon}\left(\sqrt{\frac{b_{t}}{t}}\left(x+y^{\prime}-y\right)\right) 1_{\{y \in Z[0, t]\}} \\
& =\sum_{x \in \mathbb{Z}^{2}} \sum_{y^{\prime} \in Z^{\prime}[0, t]} h_{\varepsilon}\left(\sqrt{\frac{b_{t}}{t}} x\right) \sum_{y \in \mathbb{Z}^{2}} h_{\varepsilon}\left(\sqrt{\frac{b_{t}}{t}}(x-y)\right) 1_{\left\{y+y^{\prime} \in Z[0, t]\right\}} \\
& =\sum_{y \in \mathbb{Z}^{2}} \sum_{x \in \mathbb{Z}^{2}} h_{\varepsilon}\left(\sqrt{\frac{b_{t}}{t}} x\right) h_{\varepsilon}\left(\sqrt{\frac{b_{t}}{t}}(x-y)\right)\left|Z^{\prime}[0, t] \cap(Z[0, t]-y)\right|
\end{aligned}
$$

and

$$
\left|Z^{\prime}[0, t] \cap(Z[0, t]-y)\right|=\left|Z[0, t] \cap\left(Z^{\prime}[0, t]+y\right)\right|
$$

Write $\gamma_{t}=t /\left[b_{t}\right]$ and $\Delta_{j}=\left[(j-1) \gamma_{t}, j \gamma_{t}\right], j=1, \cdots,\left[b_{t}\right]$. Note that

$$
\begin{gathered}
\sum_{j=1}^{\left[b_{t}\right]}\left|Z\left(\Delta_{j}\right) \cap Z^{\prime}[0, t]\right|-\sum_{1 \leq j<k \leq\left[b_{t}\right]}\left|Z\left(\Delta_{j}\right) \cap Z\left(\Delta_{k}\right) \cap Z^{\prime}[0, t]\right| \\
\leq\left|Z[0, t] \cap Z^{\prime}[0, t]\right| \leq \sum_{j=1}^{\left[b_{t}\right]}\left|Z\left(\Delta_{j}\right) \cap Z^{\prime}[0, t]\right|
\end{gathered}
$$


and similarly

$$
\begin{aligned}
& \sum_{j=1}^{\left[b_{t}\right]} \sum_{x \in \mathbb{Z}^{2}} q_{t, \varepsilon}(x)\left|Z\left(\Delta_{j}\right) \cap\left(x+Z^{\prime}[0, t]\right)\right| \\
& -\sum_{1 \leq j<k \leq\left[b_{t}\right]} \sum_{x \in \mathbb{Z}^{2}} q_{t, \varepsilon}(x)\left|Z\left(\Delta_{j}\right) \cap Z\left(\Delta_{k}\right) \cap\left(x+Z^{\prime}[0, t]\right)\right| \\
& \leq \sum_{x \in \mathbb{Z}^{2}} q_{t, \varepsilon}(x)\left|Z[0, t] \cap\left(x+Z^{\prime}[0, t]\right)\right| \\
& \leq \sum_{j=1}^{\left[b_{t}\right]} \sum_{x \in \mathbb{Z}^{2}} q_{t, \varepsilon}(x)\left|Z\left(\Delta_{j}\right) \cap\left(x+Z^{\prime}[0, t]\right)\right| .
\end{aligned}
$$

Hence,

$$
\begin{aligned}
& || Z[0, t] \cap Z^{\prime}[0, t]\left|-\sum_{x \in \mathbb{Z}^{2}} q_{t, \varepsilon}(x)\right| Z[0, t] \cap\left(x+Z^{\prime}[0, t]\right)|| \\
& \leq\left|\sum_{j=1}^{\left[b_{t}\right]}\left[\left|Z\left(\Delta_{j}\right) \cap Z^{\prime}[0, t]\right|-\sum_{x \in \mathbb{Z}^{2}} q_{t, \varepsilon}(x)\left|Z\left(\Delta_{j}\right) \cap\left(x+Z^{\prime}[0, t]\right)\right|\right]\right| \\
& +\sum_{1 \leq j<k \leq\left[b_{t}\right]}\left|Z\left(\Delta_{j}\right) \cap Z\left(\Delta_{k}\right) \cap Z^{\prime}[0, t]\right| \\
& +\sum_{1 \leq j<k \leq\left[b_{t}\right]} \sum_{x \in \mathbb{Z}^{2}} q_{t, \varepsilon}(x)\left|Z\left(\Delta_{j}\right) \cap Z\left(\Delta_{k}\right) \cap\left(x+Z^{\prime}[0, t]\right)\right| .
\end{aligned}
$$

We first take care of the last two terms. This is the easy step. Write

$$
\begin{aligned}
& \eta(t, \varepsilon)=\sum_{1 \leq j<k \leq\left[b_{t}\right]}\left|Z\left(\Delta_{j}\right) \cap Z\left(\Delta_{k}\right) \cap Z^{\prime}[0, t]\right| \\
& +\sum_{1 \leq j<k \leq\left[b_{t}\right]} \sum_{x \in \mathbb{Z}^{2}} q_{t, \varepsilon}(x)\left|Z\left(\Delta_{j}\right) \cap Z\left(\Delta_{k}\right) \cap\left(x+Z^{\prime}[0, t]\right)\right| .
\end{aligned}
$$

It follows from (4.2) that

$$
\sup _{t, j, k, x} \mathbb{E} \exp \left\{c \frac{(\log t)^{3 / 2}}{\sqrt{t}}\left|Z\left(\Delta_{j}\right) \cap Z\left(\Delta_{k}\right) \cap\left(x+Z^{\prime}[0, t]\right)\right|^{1 / 2}\right\}<\infty .
$$


for some $c>0$. Hence, if $b_{t}=o\left((\log t)^{1 / 5}\right)$, then for any $\theta>0$ we can find $t_{0}<\infty$ such that

$$
\begin{aligned}
& \sup _{t \geq t_{0}} \mathbb{E} \exp \left\{\theta \sqrt{\frac{b_{t}}{t}}(\log t) \eta(t, \varepsilon)^{1 / 2}\right\} \\
& \quad \leq \sup _{t \geq t_{0}} \sup _{j, k, x} \mathbb{E} \exp \left\{\theta \sqrt{\frac{b_{t}}{t}}(\log t) b_{t}^{2}\left|Z\left(\Delta_{j}\right) \cap Z\left(\Delta_{k}\right) \cap\left(x+Z^{\prime}[0, t]\right)\right|^{1 / 2}\right\} \\
& <\infty .
\end{aligned}
$$

Hence

$$
\limsup _{t \rightarrow \infty} \frac{1}{b_{t}} \log \mathbb{E} \exp \left\{\theta \sqrt{\frac{b_{t}}{t}}(\log t) \eta(t, \varepsilon)^{1 / 2}\right\}=0 .
$$

To handle the first term on the right hand side of (6.28) set

$$
\xi(t, \varepsilon)=\sum_{j=1}^{\left[b_{t}\right]}\left[\left|Z\left(\Delta_{j}\right) \cap Z^{\prime}[0, t]\right|-\sum_{x \in \mathbb{Z}^{2}} q_{t, \varepsilon}(x)\left|Z\left(\Delta_{j}\right) \cap\left(x+Z^{\prime}[0, t]\right)\right|\right]
$$

Using Fubini, independence and then the Cauchy-Schwarz inequality we have

$$
\begin{aligned}
& \left|\mathbb{E} \xi^{m}(t, \varepsilon)\right|= \\
& (2 \pi)^{-2 m} \mid \mathbb{E} \int_{\left([-\pi, \pi]^{2}\right)^{m}}\left(\prod_{k=1}^{m}\left[1-\widehat{q}_{t, \varepsilon}\left(\lambda_{k}\right)\right]\right) \\
& \quad \times\left.\left[\prod_{k=1}^{m} \sum_{x_{k}^{\prime} \in Z^{\prime}[0, t]} e^{i \lambda_{k} \cdot x_{k}^{\prime}}\right]\left[\prod_{k=1}^{m} \sum_{j=1}^{\left[b_{n}\right]} \sum_{x_{k} \in Z\left(\Delta_{j}\right)} e^{-i \lambda_{k} \cdot x_{k}}\right] d \lambda_{1} \cdots d \lambda_{m}\right|^{m}\left\{\int_{\left([-\pi, \pi]^{2}\right)^{m}}\left(\prod_{k=1}^{m}\left[1-\widehat{q}_{t, \varepsilon}\left(\lambda_{k}\right)\right]\right)\left|\mathbb{E} \prod_{k=1}^{m} \sum_{x_{k} \in Z[0, t]} e^{i \lambda_{k} \cdot x_{k}}\right|^{2} d \lambda_{1} \cdots d \lambda_{m}\right\}^{1 / 2} \\
& \leq(2 \pi)^{-2 m}\left\{\int_{\left([-\pi, \pi]^{2}\right)^{m}}\left(\prod_{k=1}^{m}\left[1-\widehat{q}_{t, \varepsilon}\left(\lambda_{k}\right)\right]\right)\left|\mathbb{E} \prod_{k=1}^{m} \sum_{j=1}^{\left[b_{t}\right]} \sum_{x_{k} \in Z\left(\Delta_{j}\right)} e^{i \lambda_{k} \cdot x_{k}}\right|^{2} d \lambda_{1} \cdots d \lambda_{m}\right\}^{1 / 2} \\
& \leq\left\{\mathbb{E}\left|Z[0, t] \cap Z^{\prime}[0, t]\right|^{m}\right\}^{1 / 2}\left\{\mathbb{E} \zeta^{m}(t, \varepsilon)\right\}^{1 / 2},
\end{aligned}
$$


where

$$
\zeta(t, \varepsilon)=\sum_{j, k=1}^{\left[b_{t}\right]}\left[\left|Z\left(\Delta_{j}\right) \cap Z^{\prime}\left(\Delta_{k}\right)\right|-\sum_{x \in \mathbb{Z}^{2}} q_{t, \varepsilon}(x)\left|Z\left(\Delta_{j}\right) \cap\left(x+Z^{\prime}\left(\Delta_{k}\right)\right)\right|\right]
$$

and we have used the fact that $1-\widehat{q}_{t, \varepsilon}(\lambda) \leq 1$ in the last step. Note that in the notation of (6.2),$\zeta(t, \varepsilon)=L$ with $p(x)=q_{t, \varepsilon}(x)$, so that by (6.4), for all $m \geq 1$

$$
\mathbb{E} \zeta^{m}(t, \varepsilon) \geq 0
$$

Let $\delta>0$ be fixed for a while. By Cauchy-Schwarz and then (6.34)

$$
\begin{aligned}
& \mathbb{E} \cosh \left\{\theta \sqrt{\frac{b_{t}}{t}}(\log t)|\xi(t, \varepsilon)|^{1 / 2}\right\} \\
& =\sum_{m=0}^{\infty} \frac{\theta^{2 m}}{(2 m) !}\left(\sqrt{\frac{b_{t}}{t}}(\log t)\right)^{2 m} \mathbb{E}\left|\xi^{m}(t, \varepsilon)\right| \\
& \leq \sum_{m=0}^{\infty} \frac{\theta^{2 m}}{(2 m) !}\left(\sqrt{\frac{b_{t}}{t}}(\log t)\right)^{2 m}\left\{\mathbb{E} \xi^{2 m}(t, \varepsilon)\right\}^{1 / 2} \\
& \leq\left\{\sum_{m=0}^{\infty} \frac{(\delta \theta)^{2 m}}{(2 m) !}\left(\sqrt{\frac{b_{t}}{t}}(\log t)\right)^{2 m}\left\{\mathbb{E}\left|Z[0, t] \cap Z^{\prime}[0, t]\right|^{2 m}\right\}^{1 / 2}\right\}^{1 / 2} \\
& \left.\times\left\{\sum_{m=0}^{\infty} \frac{\left(\delta^{-1} \theta\right)^{2 m}}{(2 m) !}\left(\sqrt{\frac{b_{t}}{t}}(\log t)\right)^{2 m}\left\{\mathbb{E} \zeta^{2 m}(t, \varepsilon)\right\}^{1 / 2}\right\}^{1 / 2}\right\}^{1 / 2} \\
& \leq\left\{\sum_{m=0}^{\infty} \frac{(\delta \theta)^{m}}{m !}\left(\sqrt{\frac{b_{t}}{t}}(\log t)\right)^{m}\left\{\mathbb{E}\left|Z[0, t] \cap Z^{\prime}[0, t]\right|^{m}\right\}^{1 / 2}\right\}^{1 / 2} \\
& \times\left\{\sum_{m=0}^{\infty} \frac{\left(\delta^{-1} \theta\right)^{m}}{m !}\left(\sqrt{\frac{b_{t}}{t}}(\log t)\right)^{m}\left\{\mathbb{E} \zeta^{m}(t, \varepsilon)\right\}^{1 / 2}\right\}^{1 / 2},
\end{aligned}
$$

where in the last step we used ([6.36) and the fact that $\left|Z[0, t] \cap Z^{\prime}[0, t]\right| \geq 0$.

By [8. (2.11)], there is a $C>0$ independent of $\delta$ and $\theta$ such that (6.38)

$$
\lim _{t \rightarrow \infty} \frac{1}{b_{t}} \log \sum_{m=0}^{\infty} \frac{(\delta \theta)^{m}}{m !}\left(\sqrt{\frac{b_{t}}{t}}(\log t)\right)^{m}\left\{\mathbb{E}\left|Z[0, t] \cap Z^{\prime}[0, t]\right|^{m}\right\}^{1 / 2}=C(\delta \theta)^{2} .
$$


In addition, by Lemma 6.1

$$
\begin{gathered}
\sum_{m=0}^{\infty} \frac{\left(\delta^{-1} \theta\right)^{m}}{m !}\left(\sqrt{\frac{b_{t}}{t}}(\log t)\right)^{m}\left\{\mathbb{E} \zeta^{m}(t, \varepsilon)\right\}^{1 / 2} \\
\leq\left\{\sum_{m=0}^{\infty} \frac{\left(\delta^{-1} \theta\right)^{m}}{m !}\left(\sqrt{\frac{b_{t}}{t}}(\log t)\right)^{m}\left\{\mathbb{E}|\beta(t, \varepsilon)|^{m}\right\}^{1 / 2}\right\}^{\left[b_{t}\right]},
\end{gathered}
$$

where

$$
\beta(t, \varepsilon)=\left|Z\left[0, \gamma_{t}\right] \cap Z^{\prime}\left[0, \gamma_{t}\right]\right|-\sum_{x \in \mathbb{Z}^{2}} q_{t, \varepsilon}(x)\left|Z\left[0, \gamma_{t}\right] \cap\left(x+Z^{\prime}\left[0, \gamma_{t}\right]\right)\right|
$$

Recall that $q_{t, \varepsilon}(x)$ is defined by (6.22) and $\gamma_{t}=t /\left[b_{t}\right]$. As in the proof of (6.23) we can check that

$$
\sum_{x \in \mathbb{Z}^{2}} q_{t, \varepsilon}(x)\left|Z\left[0, \gamma_{t}\right] \cap\left(x+Z^{\prime}\left[0, \gamma_{t}\right]\right)\right|=B_{\gamma_{t}, 1}
$$

see (5.2). By Lemma 5.1 (with $t$ replaced by $\gamma_{t}$ ),

$$
\frac{b_{t}(\log t)^{2}}{t} \beta(t, \varepsilon) \stackrel{d}{\longrightarrow}(2 \pi)^{2} \operatorname{det}(\Gamma)\left[\alpha\left([0,1]^{2}\right)-\alpha_{\varepsilon}\left([0,1]^{2}\right)\right] .
$$

By Lemma 4.1 (with $p=2$ ),

$$
\mathbb{E}|\beta(t, \varepsilon)|^{m} \leq 2 \sup _{x} \mathbb{E}^{(0, x)}\left|Z\left[0, \gamma_{t}\right] \cap Z^{\prime}\left[0, \gamma_{t}\right]\right|^{m} \leq m ! C^{m}\left(\frac{t}{b_{t}}(\log t)^{-2}\right)^{m}
$$

Hence,

$$
\begin{gathered}
\lim _{t \rightarrow \infty} \sum_{m=0}^{\infty} \frac{\left(\delta^{-1} \theta\right)^{m}}{m !}\left(\sqrt{\frac{b_{t}}{t}}(\log t)\right)^{m}\left\{\mathbb{E}|\beta(t, \varepsilon)|^{m}\right\}^{1 / 2} \\
=\sum_{m=0}^{\infty} \frac{\left(\delta^{-1} \theta\right)^{m}}{m !}((2 \pi) \sqrt{\operatorname{det}(\Gamma)})^{m}\left\{\mathbb{E}\left|\alpha\left([0,1]^{2}\right)-\alpha_{\varepsilon}\left([0,1]^{2}\right)\right|^{m}\right\}^{1 / 2} .
\end{gathered}
$$

So by (6.39) we have

$$
\begin{aligned}
& \text { 44) } \lim \sup _{t \rightarrow \infty} \frac{1}{b_{t}} \log \sum_{m=0}^{\infty} \frac{\left(\delta^{-1} \theta\right)^{m}}{m !}\left(\sqrt{\frac{b_{t}}{t}}(\log t)\right)^{m}\left\{\mathbb{E} \zeta^{m}(t, \varepsilon)\right\}^{1 / 2} \\
& \leq \log \sum_{m=0}^{\infty} \frac{\left(\delta^{-1} \theta\right)^{m}}{m !}((2 \pi) \sqrt{\operatorname{det}(\Gamma)})^{m}\left\{\mathbb{E}\left|\alpha\left([0,1]^{2}\right)-\alpha_{\varepsilon}\left([0,1]^{2}\right)\right|^{m}\right\}^{1 / 2} .
\end{aligned}
$$


By [24, Theorem 1, p.183],

$$
\mathbb{E}\left|\alpha\left([0,1]^{2}\right)-\alpha_{\varepsilon}\left([0,1]^{2}\right)\right|^{m} \longrightarrow 0 \quad \text { as } \varepsilon \rightarrow 0^{+},
$$

for all $m \geq 1$. In addition, by [7, (1.12)], there is a constant $C>0$ such that

$$
\mathbb{E}\left|\alpha\left([0,1]^{2}\right)-\alpha_{\varepsilon}\left([0,1]^{2}\right)\right|^{m} \leq \mathbb{E} \alpha^{m}\left([0,1]^{2}\right) \leq m ! C^{m}
$$

for all $m \geq 1$. By dominated convergence, therefore,

$$
\sum_{m=0}^{\infty} \frac{\left(\delta^{-1} \theta\right)^{m}}{m !}((2 \pi) \sqrt{\operatorname{det}(\Gamma)})^{m}\left\{\mathbb{E}\left|\alpha\left([0,1]^{2}\right)-\alpha_{\varepsilon}\left([0,1]^{2}\right)\right|^{m}\right\}^{1 / 2} \longrightarrow 1
$$

as $\varepsilon \rightarrow 0^{+}$. (Alternatively, this follows immediately from [12, (6.29)]). Thus

$$
\lim _{\varepsilon \rightarrow 0^{+}} \limsup _{t \rightarrow \infty} \frac{1}{b_{t}} \log \sum_{m=0}^{\infty} \frac{\left(\delta^{-1} \theta\right)^{m}}{m !}\left(\sqrt{\frac{b_{t}}{t}}(\log t)\right)^{m}\left\{\mathbb{E} \zeta^{m}(t, \varepsilon)\right\}^{1 / 2}=0
$$

Summarizing what we have,

$$
\limsup _{\varepsilon \rightarrow 0^{+}} \limsup _{t \rightarrow \infty} \frac{1}{b_{t}} \log \mathbb{E} \cosh \left\{\theta \sqrt{\frac{b_{t}}{t}}(\log t)|\xi(t, \varepsilon)|^{1 / 2}\right\} \leq C(\delta \theta)^{2} .
$$

Letting $\delta \rightarrow 0^{+}$gives

$$
\limsup _{\varepsilon \rightarrow 0^{+}} \limsup _{t \rightarrow \infty} \frac{1}{b_{t}} \log \mathbb{E} \cosh \left\{\theta \sqrt{\frac{b_{t}}{t}}(\log t)|\xi(t, \varepsilon)|^{1 / 2}\right\}=0 .
$$

Since $\exp (x) \leq 2 \cosh (x)$ we see from (6.50) that

$$
\limsup _{\varepsilon \rightarrow 0^{+}} \limsup _{t \rightarrow \infty} \frac{1}{b_{t}} \log \mathbb{E} \exp \left\{\theta \sqrt{\frac{b_{t}}{t}}(\log t)|\xi(t, \varepsilon)|^{1 / 2}\right\}=0 .
$$

By (6.28) and (6.23) we have thus completed the proof of Lemma 4.7 when $j=0$. If in (4.22) with $j=0$ we replace $t$ by $2^{-j} t, \theta$ by $2^{-j / 2} \theta, b_{t}$ by $\widetilde{b}_{t}=: b_{2^{j} t}$ and $\varepsilon$ by $2^{j / 2} \varepsilon$, we obtain (4.22) for any $j$ (compare the proof of (4.20) ). 


\section{Laws of the iterated logarithm}

We first prove some lemmas in preparation for the proof of Theorem 1.6. Define

$$
\widetilde{\varphi}_{j}=\frac{j}{\mathcal{H}(j)}, \quad \widetilde{G}_{j}=\left(R_{j}-\widetilde{\varphi}_{j}\right) \frac{\log ^{2} n}{n},
$$

and $K=[\log \log n]+1$.

Lemma 7.1 There exists a constant $c_{1}$ such that if $A$ and $B$ are positive integers and $C=A+B$, then

$$
\left|\widetilde{\varphi}_{C}-\widetilde{\varphi}_{A}-\widetilde{\varphi}_{B}\right| \leq c_{1} \frac{(A \wedge B)^{1 / 2}}{C^{1 / 2} \log ^{2} C} .
$$

Proof. The cases when $A$ or $B$ equal 1 are easy, so we suppose $A, B>1$. Write

$$
\widetilde{\varphi}_{C}-\widetilde{\varphi}_{A}-\widetilde{\varphi}_{B}=\frac{C}{\mathcal{H}(C)}\left[-\frac{A}{C} \frac{\mathcal{H}(C)-\mathcal{H}(A)}{\mathcal{H}(A)}-\frac{B}{C} \frac{\mathcal{H}(C)-\mathcal{H}(B)}{\mathcal{H}(B)} .\right]
$$

By (2.2) and (2.3), the right hand side is bounded in absolute value by

$$
c_{2} \frac{C}{\log C}\left[-\frac{A}{C} \frac{\log C-\log A}{\log A}-\frac{B}{C} \frac{\log C-\log B}{\log B}\right]=c_{2}\left|\varphi_{C}-\varphi_{A}-\varphi_{B}\right|,
$$

where $\varphi_{j}=j / \log j$. Our result now follows by Lemma 4.2 of [6].

Lemma 7.2 There exists $\lambda_{0}$ such that if $\lambda \geq \lambda_{0}$, then

$$
\mathbb{P}\left(\max _{m \leq n} \bar{R}_{m}>\lambda n \log \log \log n / \log ^{2} n\right) \leq(\log n)^{-2} .
$$

Proof. Using Lemma 7.1 in place of Lemma 4.2 of [6] and with $\widetilde{\varphi}_{j}, \widetilde{G}_{j}$ replacing $\varphi_{j}, G_{j}$, resp., we have by [6], Lemma 4.3 and the proof of Proposition 4.1 (up through the display in the middle of p. 1390), that

$$
\mathbb{P}\left(\max _{m \leq n} \widetilde{G}_{m}>A \log \log \log n\right) \leq(\log n)^{-2}
$$


if $A$ is large enough. By (2.1) and (2.2), we see that

$$
\max _{m \leq n}\left|\bar{R}_{m}-\left(R_{m}-\widetilde{\varphi}_{m}\right)\right| \leq c_{1} \frac{n}{\log ^{2} n}=o\left(n \log \log \log n / \log ^{2} n\right),
$$

and our result now follows immediately.

Proof of Theorem 1.6; Let $\xi=2 \pi \sqrt{\operatorname{det} \Gamma}$. We begin with the upper bound. Let $\eta, \varepsilon>0$ be small and let $q>1$ be very close to 1 . Let $t_{i}=\left[q^{i}\right]$. If

$$
A_{i}=\left\{\bar{R}_{t_{i}} \geq(1+\eta) \xi t_{i} \log \log \log t_{i} / \log ^{2} t_{i}\right\},
$$

then it follows from Theorem 1.1 that $\sum_{i} \mathbb{P}\left(A_{i}\right)<\infty$, and so by BorelCantelli, $\mathbb{P}\left(A_{i}\right.$ i.o. $)=0$.

Next, if $\lambda$ is sufficiently large,

$$
\mathbb{P}\left(\max _{m \leq n} \bar{R}_{m}>\lambda n \log \log \log n / \log ^{2} n\right) \leq(\log n)^{-2} ;
$$

by Lemma 7.2, Let

$$
B_{i}=\left\{\max _{t_{i} \leq k \leq t_{i+1}}\left[\bar{R}_{k}-\bar{R}_{t_{i}}\right]>\varepsilon t_{i} \log \log \log t_{i} / \log ^{2} t_{i}\right\} .
$$

By subadditivity $R_{k}-R_{t_{i}} \leq R_{k-t_{i}} \circ \theta_{t_{i}}$, where $\theta_{t_{i}}$ is the usual shift operator of Markov theory. By Lemma 7.1]

$$
\mathbb{E} R_{k}-\mathbb{E} R_{t_{i}} \geq \mathbb{E} R_{k-t_{i}}-c \frac{t_{i}}{\log ^{2} t_{i}} .
$$

So by the Markov property, and using the fact that the $\mathbb{P}^{x}$ law of $R_{k-t_{i}}$ does not depend on $x$, for $i$ large

$$
\begin{aligned}
& \mathbb{P}\left(B_{i}\right) \\
& =\mathbb{P}\left(\max _{t_{i} \leq k \leq t_{i+1}}\left[R_{k}-R_{t_{i}}-\left(\mathbb{E} R_{k}-\mathbb{E} R_{t_{i}}\right)\right]>\varepsilon t_{i} \log \log \log t_{i} / \log ^{2} t_{i}\right) \\
& \leq \mathbb{P}\left(\max _{t_{i} \leq k \leq t_{i+1}}\left[R_{k}-R_{t_{i}}-\mathbb{E} R_{k-t_{i}}\right]+c \frac{t_{i}}{\log ^{2} t_{i}}>\varepsilon t_{i} \log \log \log t_{i} / \log ^{2} t_{i}\right) \\
& \leq \mathbb{P}^{S_{t_{i}}}\left(\max _{t_{i} \leq k \leq t_{i+1}}\left[\bar{R}_{k-t_{i}}\right]>\varepsilon t_{i} \log \log \log t_{i} / \log ^{2} t_{i}-c \frac{t_{i}}{\log ^{2} t_{i}}\right) \\
& \leq \mathbb{P}\left(\max _{k \leq t_{i+1}-t_{i}} \bar{R}_{k} \geq \frac{\varepsilon}{2} t_{i} \log \log \log t_{i} / \log ^{2} t_{i}\right)
\end{aligned}
$$


If $q$ is sufficiently small, then $\sum_{i} \mathbb{P}\left(B_{i}\right)$ will be summable by (17.1). So with probability one, for $i$ large enough

$$
\max _{k \leq t_{i+1}} \bar{R}_{k} \leq((1+\eta) \xi+\varepsilon) q t_{i} \log \log \log t_{i} / \log ^{2} t_{i}
$$

Since $\eta$ and $\varepsilon$ are arbitrary, and we can take $q$ as close to 1 as we like, this implies the upper bound.

Let $\eta>0, t_{i}=\left[\exp \left(i^{1+\frac{\eta}{2}}\right)\right], V_{i}=\# S\left(\left(t_{i}, t_{i+1}\right]\right)$, and set

$$
C_{i}=\left\{\bar{V}_{i}>(1-\eta) \xi\left(t_{i+1}-t_{i}\right) \log \log \log \left(t_{i+1}-t_{i}\right) / \log ^{2}\left(t_{i+1}-t_{i}\right)\right\} .
$$

Note that the events $C_{i}$ are independent. By Theorem 1.1 and Borel-Cantelli, $\mathbb{P}\left(C_{i}\right.$ i.o. $)=1$. Note

$$
\frac{\left(t_{i+1}-t_{i}\right) \log \log \log \left(t_{i+1}-t_{i}\right)}{\log ^{2}\left(t_{i+1}-t_{i}\right)}=\frac{t_{i+1} \log \log \log t_{i+1}}{\log ^{2} t_{i+1}}(1+o(1)) .
$$

Also

$$
\left|V_{i}-R_{t_{i+1}}\right|+\left|\mathbb{E} V_{i}-\mathbb{E} R_{t_{i+1}}\right| \leq 2 t_{i}=o\left(\frac{t_{i+1} \log \log \log t_{i+1}}{\log ^{2} t_{i+1}}\right) .
$$

Therefore with probability one, infinitely often

$$
\bar{R}_{t_{i+1}}>\left(1-\frac{\eta}{2}\right) \xi t_{i+1} \log \log \log t_{i+1} / \log ^{2} t_{i+1} .
$$

This proves the lower bound.

We now turn to the LIL for $-\bar{R}_{n}$. First we prove

Lemma 7.3 Let $\varepsilon>0$. There exists $q_{0}(\varepsilon)$ such that if $1<q<q_{0}(\varepsilon)$, then

$$
\mathbb{P}\left(\max _{\left[q^{-1} n\right] \leq k \leq n}\left(\bar{R}_{n}-\bar{R}_{k}\right)>\varepsilon n \log \log n / \log ^{2} n\right) \leq \frac{1}{\log ^{2} n}
$$

for $n$ large.

Proof. Let

$$
G_{k}=\left(R_{n}-R_{k}\right) \frac{\log ^{2} n}{n} .
$$


Let

$$
\mathcal{A}_{i}=\left\{\left[q^{-1} n\right]+\left[\frac{n \ell}{2^{i}}\right]: \ell \in \mathbb{Z}_{+}\right\} \cap[0, n], \quad i \leq \log _{2} n+1 .
$$

Given $k$, let $k_{i}=\max \left\{j \in \mathcal{A}_{i}: j \leq k\right\}$. We write

$$
G_{k}=G_{k_{1}}+\left(G_{k_{2}}-G_{k_{1}}\right)+\left(G_{k_{3}}-G_{k_{2}}\right)+\cdots
$$

where the sum is actually a finite one. If $\bar{G}_{k}>\varepsilon \log \log n$ for some $\left[q^{-1} n\right] \leq$ $k \leq n$, then either

$$
\bar{G}_{\left[q^{-1} n\right]}>\frac{\varepsilon}{2} \log \log n
$$

or for some $i$ there exist consecutive elements $\ell, m$ of $\mathcal{A}_{i}$ such that

$$
\bar{G}_{m}-\bar{G}_{\ell}>\frac{\varepsilon}{10 i^{2}} \log \log n .
$$

By subadditivity $R_{n}-R_{k} \leq R_{n-k} \circ \theta_{k}$ for $k \leq n$, while by Lemma [7.1

$$
\mathbb{E} R_{n}-\mathbb{E} R_{k} \geq \mathbb{E} R_{n-k}-c_{1}\left(1-q^{-1}\right)^{1 / 2} \frac{n}{\log ^{2} n} .
$$

Then setting $k=\left[q^{-1} n\right]$,

$$
\begin{aligned}
& \mathbb{P}\left(\bar{G}_{\left[q^{-1} n\right]}>\frac{\varepsilon}{2} \log \log n\right) \\
& \quad=\mathbb{P}\left(\left(R_{n}-R_{k}\right) \frac{\log ^{2} n}{n}-\left(\mathbb{E} R_{n}-\mathbb{E} R_{k}\right) \frac{\log ^{2} n}{n}>\frac{\varepsilon}{2} \log \log n\right) \\
& \quad \leq \mathbb{P}^{S_{k}}\left(R_{n-k} \frac{\log ^{2} n}{n}-\mathbb{E} R_{n-k} \frac{\log ^{2} n}{n}+c_{1}\left(1-q^{-1}\right)^{1 / 2}>\frac{\varepsilon}{2} \log \log n\right) .
\end{aligned}
$$

Using the fact that the $\mathbb{P}^{x}$ law of $R_{n-k}$ does not depend on $x$, this is the same as

$$
\mathbb{P}\left(\frac{\bar{R}_{n-k}}{(n-k) / \log ^{2}(n-k)}>\frac{n}{n-k} \frac{\log ^{2}(n-k)}{\log ^{2} n}\left(\frac{\varepsilon}{2} \log \log n-c_{1}\left(1-q^{-1}\right)^{1 / 2}\right)\right) .
$$

If $q>1$ is close enough to 1 and $n$ is large enough, by Theorem 1.5 this is bounded by

$$
c_{2} \exp \left(-c_{3} \frac{\varepsilon}{2} \frac{1}{1-q^{-1}} \log \log n\right) \leq \frac{1}{2 \log ^{2} n}
$$


This bounds the probability of the event described in (7.4).

Similarly, $R_{m}-R_{\ell} \leq R_{m-\ell} \circ \theta_{\ell}$ and by Lemma 7.1

$$
\mathbb{E} R_{m}-\mathbb{E} R_{\ell} \geq \mathbb{E} R_{m-\ell}-c_{1}\left(\frac{m-\ell}{n}\right)^{1 / 2} \frac{n}{\log ^{2} n} .
$$

So if $\ell$ and $m$ are consecutive elements of $\mathcal{A}_{i}$, similarly to (7.6) we obtain

$$
\mathbb{P}\left(\bar{G}_{m}-\bar{G}_{\ell} \geq \frac{\varepsilon}{10 i^{2}} \log \log n\right) \leq \mathbb{P}\left(\frac{\bar{R}_{m-\ell}}{n / \log ^{2} n} \geq \frac{\varepsilon}{10 i^{2}} \log \log n-c_{1} 2^{-i / 2}\right) .
$$

For $n$ large, $c_{1} 2^{-i / 2} \leq \frac{\varepsilon}{20 i^{2}} \log \log n$ for all $i$ and $n /(m-\ell)=2^{i}$, so by Theorem 1.5 the left hand side of (7.7) is less than

$$
\mathbb{P}\left(\frac{\bar{R}_{m-\ell}}{(m-\ell) / \log ^{2}(m-\ell)} \geq \frac{\varepsilon}{40 i^{2}} \frac{n}{m-\ell} \log \log n\right) \leq c_{2} \exp \left(-c_{3} \frac{\log \log n}{40 i^{2}} 2^{i}\right) .
$$

There are at most $2^{i+1}$ such pairs $\ell, m$, so

$w_{i}:=\mathbb{P}\left(\right.$ for some consecutive elements $\left.\ell, m \in \mathcal{A}_{i}: \bar{G}_{m}-\bar{G}_{\ell}>\frac{\varepsilon}{10 i^{2}} \log \log n\right)$

$$
\leq c_{2} 2^{i+1} \exp \left(-c_{3} \frac{\log \log n}{40 i^{2}} 2^{i}\right)
$$

Since $c_{3} 2^{i} / 40 i^{2}>2(i+1) \log 2$ for $i$ large, then for $n$ large enough

$$
w_{i} \leq c_{2} \exp \left(-c_{3} \frac{2^{i} \log \log n}{40 i^{2}}\right)
$$

So then

$$
\sum_{i=1}^{\infty} w_{i} \leq \frac{1}{2 \log ^{2} n}
$$

for large $n$, and this bounds the event that for some $i$ there exist consecutive elements $\ell, m$ of $\mathcal{A}_{i}$ such that (7.5) holds. Combining with the bound for (17.4), the result follows.

Proof of Theorem 1.7; Let

$$
\Theta=(2 \pi)^{2} \operatorname{det}(\Gamma)^{-1 / 2} \kappa(2,2)^{-4}
$$


Upper bound. Let $\eta, \varepsilon>0$ and choose $q \in\left(1, q_{0}(\varepsilon)\right)$ where $q_{0}(\varepsilon)$ is as in Lemma 7.3, Let $t_{i}=\left[q^{i}\right]$. If

$$
A_{i}=\left\{-\bar{R}_{t_{i}}>(1+\eta) \Theta^{-1} \frac{t_{i} \log \log t_{i}}{\log ^{2} t_{i}}\right\},
$$

then by Theorem 1.5. $\sum_{i} \mathbb{P}\left(A_{i}\right)<\infty$, and hence by Borel-Cantelli, $\mathbb{P}\left(A_{i}\right.$ i.o. $)=$ 0 . Let

$$
B_{i}=\left\{\max _{t_{i} \leq k \leq t_{i+1}}\left(\bar{R}_{t_{i+1}}-\bar{R}_{k}\right)>\varepsilon \frac{t_{i+1} \log \log t_{i+1}}{\log ^{2} t_{i+1}}\right\} .
$$

By Lemma 7.3, $\sum_{i} \mathbb{P}\left(B_{i}\right)<\infty$, and again $\mathbb{P}\left(B_{i}\right.$ i.o. $)=0$. So with probability one, for $k$ large we have $t_{i} \leq k \leq t_{i+1}$ for some $i$ large, and then

$$
\begin{aligned}
-\bar{R}_{k} & =-\bar{R}_{t_{i+1}}+\left(\bar{R}_{t_{i+1}}-\bar{R}_{k}\right) \\
& \leq \Theta^{-1}(1+\eta) \frac{t_{i+1} \log \log t_{i+1}}{\log ^{2} t_{i+1}}+\varepsilon \frac{t_{i+1} \log \log t_{i+1}}{\log ^{2} t_{i+1}} \\
& \leq q\left(\Theta^{-1}(1+2 \eta)+2 \varepsilon\right) \frac{k \log \log k}{\log ^{2} k} .
\end{aligned}
$$

Since $\varepsilon, \eta$ can be made as small as we like and we can take $q$ as close to 1 as we like, this gives the upper bound.

Lower bound. Let $\eta>0, t_{i}=\left[\exp \left(i^{1+\frac{\eta}{2}}\right)\right], V_{i}=\# S\left(\left(t_{i}, t_{i+1}\right]\right)$. Let

$$
C_{i}=\left\{-\bar{V}_{i} \geq \Theta^{-1}(1-\eta) \frac{\left(t_{i+1}-t_{i}\right) \log \log \left(t_{i+1}-t_{i}\right)}{\log ^{2}\left(t_{i+1}-t_{i}\right)}\right\} .
$$

By Theorem 1.5. $\sum_{i} \mathbb{P}\left(C_{i}\right)=\infty$. The $C_{i}$ are independent, and so by BorelCantelli, $\mathbb{P}\left(C_{i}\right.$ i.o. $)=1$.

Since $R_{t_{i+1}} \leq V_{i}+R_{t_{i}}$ and $\mathbb{E} R_{t_{i+1}} \geq \mathbb{E} V_{i}$, then

$$
-\bar{R}_{t_{i+1}} \geq-\bar{V}_{i}-R_{t_{i}}
$$

Now

$$
R_{t_{i}} \leq t_{i}=o\left(\frac{t_{i+1} \log \log t_{i+1}}{\log ^{2} t_{i+1}}\right)
$$

and

$$
\frac{\left(t_{i+1}-t_{i}\right) \log \log \left(t_{i+1}-t_{i}\right)}{\log ^{2}\left(t_{i+1}-t_{i}\right)} \sim \frac{t_{i+1} \log \log t_{i+1}}{\log ^{2} t_{i+1}},
$$


SO

$$
-\bar{R}_{t_{i+1}} \geq \Theta^{-1}(1-2 \eta) \frac{t_{i+1} \log \log t_{i+1}}{\log ^{2} t_{i+1}}, \quad \text { i.o. }
$$

This implies the lower bound.

\section{References}

1. R.F. Bass, Probabilistic Techniques in Analysis, Springer, New York, 1995.

2. R.F. Bass and X. Chen, Self intersection local time: critical exponent, large deviations and law of the iterated logarithm. Ann. Probab. 32 (2004) 3221-3247.

3. R. Bass, X. Chen and J. Rosen, Large deviations for renormalized selfintersection local times of stable processes, Ann. Probab., 33 (2005) 984-1013.

4. R.F. Bass, X. Chen, and J. Rosen, Moderate deviations and laws of the iterated logarithm for the renormalized self-intersection local times of planar random walks, preprint.

5. R.F. Bass and D. Khoshnevisan, Intersection local times and Tanaka formulas, Ann. Inst. H. Poincaré Prob. Stat. 29 (1993) 419-452.

6. R.F. Bass and T. Kumagai, Laws of the iterated logarithm for the range of random walks in two and three dimensions. Ann. Probab. 30 (2002) 1369-1396.

7. X. Chen, Exponential asymptotics and law of the iterated logarithm for intersection local times of random walks. Ann. Probab. 32 (2004) 3248-3300.

8. X. Chen, Moderate deviations and law of the iterated logarithm for intersections of the range of random walks. Ann. Probab. 33 (2005) 10141059 .

9. X. Chen, Moderate and small deviations for the ranges of onedimensional random walks. J. Theor. Probab., to appear. 
10. X. Chen and W. Li, Large and moderate deviations for intersection local times. Probab. Theor. Rel. Fields 128 (2004) 213-254.

11. X. Chen, W. Li and J. Rosen, Large deviations for local times of stable processes and stable random walks in 1 dimension, Electron. J. Prob. 10 (2005) 577-608.

12. X. Chen and J. Rosen, Exponential asymptotics for intersection local times of stable processes, Ann. Inst. Henri Poincaré, 41 (2005) 901-928.

13. A. Dembo and O. Zeitouni, Large Deviations Techniques and Applications. (2nd ed.), Springer, New York, 1998.

14. M. Donsker and S.R.S. Varadhan, On the number of distinct sites visited by a random walk. Comm. Pure Appl. Math. 32 (1979) 721-747.

15. A. Dvoretzky and P. Erdös, Some problems on random walk in space. Proceedings of the Second Berkeley Symposium on Mathematical Statistics and Probability, 1950, 353-367. University of California Press, Berkeley and Los Angeles, 1951.

16. E. B. Dynkin, Self-intersection gauge for random walks and for Brownian motion, Ann. Probab. 16 (1988) 1-57.

17. E. Giné and V. de la Peña, Decoupling, Springer-Verlag, Berlin, 1999.

18. Y. Hamana and H. Kesten, Large deviations for the range of an integer valued random walk. Ann. Inst. H. Poincaré. 38 (2002) 17-58.

19. Y. Hamana and H. Kesten, A large-deviation result for the range of random walk and for the Wiener sausage. Probab. Theory Rel. Fields 120 (2001) 183-208.

20. N.C. Jain and W.E. Pruitt, The range of recurrent random walk in the plane. Z. Wahrschein. v. Gebiete 16 (1970) 279-292.

21. J.-F. Le Gall. Proprietés d'intersection des marches aléatoires. Comm. Math. Phys. 104 (1986) 471-507.

22. J.-F. Le Gall. Fluctuation results for the Wiener sausage. Ann. Probab. 16 (1988) 991-1018. 
23. J.-F. Le Gall and J. Rosen, The range of stable random walks. Ann. Probab. 19 (1991) 650-705.

24. J.-F. LeGall. Some properties of planar Brownian motion, Ecole d'été de probabilités de St. Flour XX, 1990), Springer-Verlag, Berlin, 1992.

25. M. Ledoux and M. Talagrand, Probability in Banach Spaces, SpringerVerlag, Berlin, 1991.

26. M. Marcus and J. Rosen, Laws of the iterated logarithm for the local times of symmetric Lévy processes and recurrent random walks. Ann. Probab. 22 (1994) 626-659.

27. M. Marcus and J. Rosen, Laws of the iterated logarithm for the local times of recurrent random walks on $\mathbb{Z}^{2}$ and of Lévy processes and random walks in the domain of attraction of Cauchy random variables. Ann. Inst. Henri Poincaré 30 (1994) 467-499.

28. M. Marcus and J. Rosen, Renormalized self-intersection local times and Wick power chaos processes, Memoirs of the AMS, (1999), Volume 142, Number 675.

29. M. Marcus and J. Rosen, Joint continuity of renormalized intersection local times, Ann. Inst. H. Poincaré Prob. Stat. 32 (1996) 671-700.

30. J. Rosen. Joint continuity and a Doob-Meyer type decomposition for renormalized intersection local times. Ann. Inst. Henri Poincaré , 35 (1999), 143-176.

31. J. Rosen. Continuity and singularity of the intersection local time of stable processes in $\mathbb{R}^{2}$. Ann. Probab. 16 (1988) 75-79.

32. J. Rosen. The asymptotics of stable sausages in the plane. Ann. Probab. 20 (1992) 29-60.

33. J. Rosen, Random walks and intersection local time. Ann. Probab. 18 (1990) 959-977.

34. F. Spitzer, Principles of Random Walk. Springer, Berlin, 1976. 
35. S. R. S. Varadhan. Appendix to Euclidian quantum field theory by K. Symanzyk. In R. Jost, editor, Local Quantum Theory. Academic Press, 1969.

Richard Bass

Department of Mathematics

University of Connecticut

Storrs, CT 06269-3009

bass@math.uconn.edu

\section{Xia Chen}

Department of Mathematics

University of Tennessee

Knoxville, TN 37996-1300

xchen@math.utk.edu

Jay Rosen

Department of Mathematics

College of Staten Island, CUNY

Staten Island, NY 10314

jrosen3@earthlink.net 